\author{
Aus dem Fachbereich Medizin \\ der Johann Wolfgang Goethe-Universität \\ Frankfurt am Main
}

betreut am

Zentrum der Chirurgie

Klinik für Thorax-, Herz- und Thorakale Gefäßchirurgie

Direktor: Prof. Dr. Thomas Walther

\title{
Veränderungen des Herzrhythmus nach minimalinvasiver Mitralklappenchirurgie
}

\author{
Dissertation \\ zur Erlangung des Doktorgrades der Medizin \\ des Fachbereichs Medizin \\ der Johann Wolfgang Goethe-Universität \\ Frankfurt am Main
}

vorgelegt von

Svenja Gehlen

aus Freiburg im Breisgau

Frankfurt am Main, 2020 
Dekan:

Referent:

Korreferent/in:

Tag der mündlichen Prüfung:
Prof. Dr. Stefan Zeuzem

Prof. Dr. Anton Moritz

Prof. Dr. Thomas Walther

26.04.2021 


\section{Inhaltsverzeichnis}

1 Abkürzungsverzeichnis, Abbildungsverzeichnis, Tabellenverzeichnis...... 7

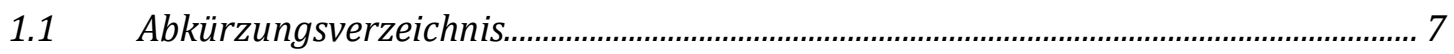

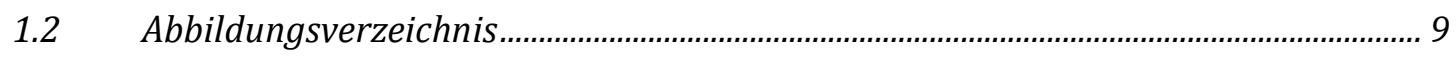

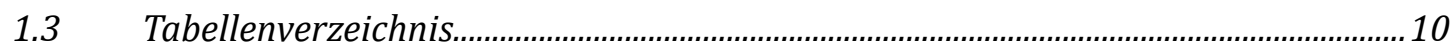

2 Einleitung

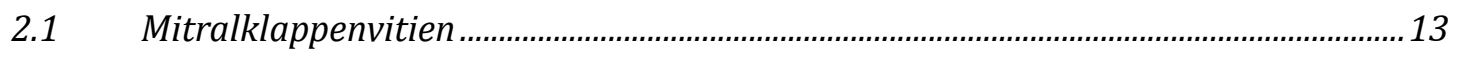

2.1.1 Anatomie der Mitralklappe ....................................................................................13

2.1.2 Mitralklappeninsuffizienz ....................................................................................14

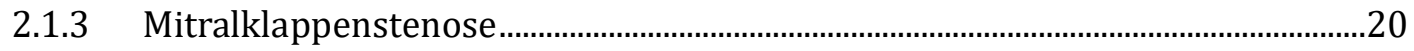

2.1.4 Minimalinvasive Mitralklappenchirurgie ……….......................................................22

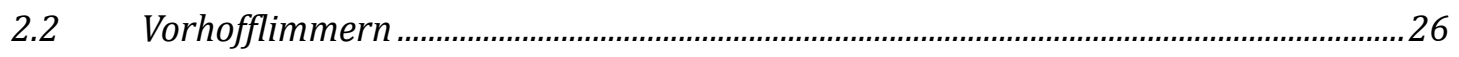

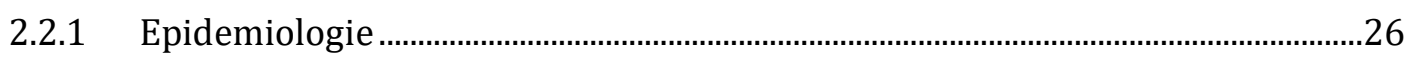

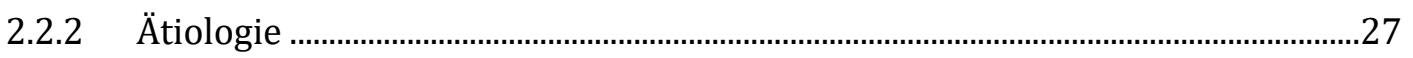

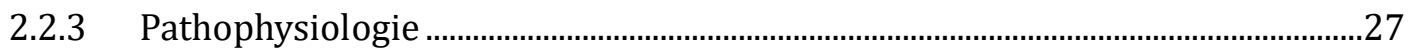

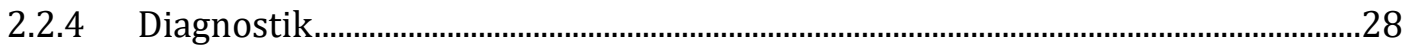

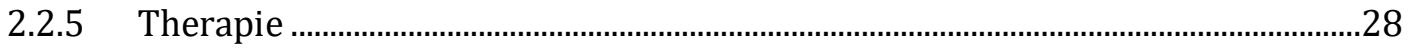

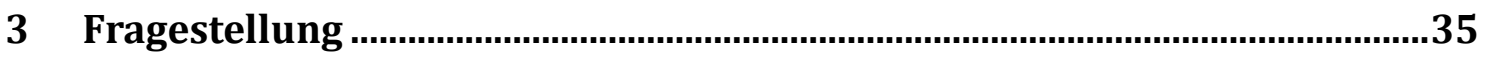

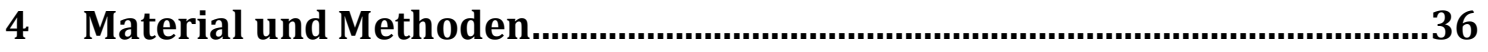

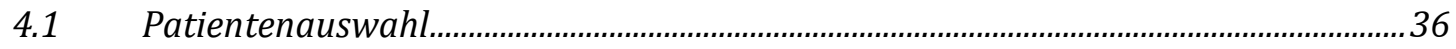

$4.2 \quad$ Datenerhebung

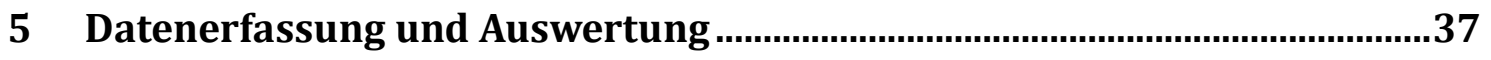

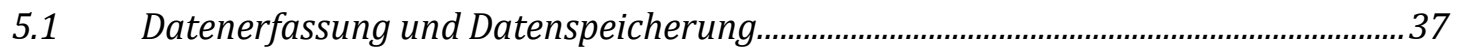

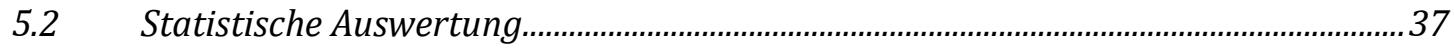


6 Ergebnisse

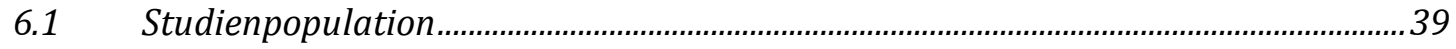

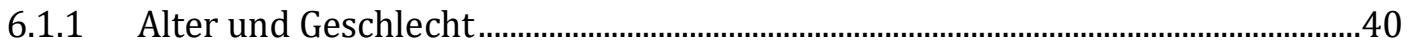

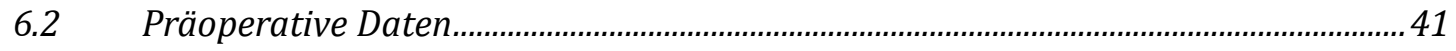

6.2.1 Kardialer präoperativer Status ...............................................................................

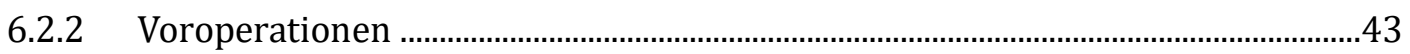

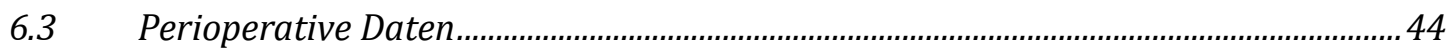

6.3.1 Art des Eingriffs und Kombinationseingriffe .........................................................44

6.3.2 Perioperative Schrittmacherimplantationen ..............................................................46

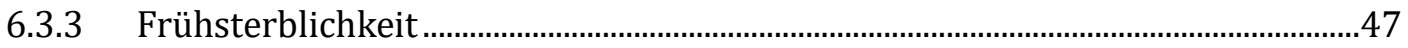

6.4 Postoperative Daten (= zum Zeitpunkt der Entlassung) .............................................48

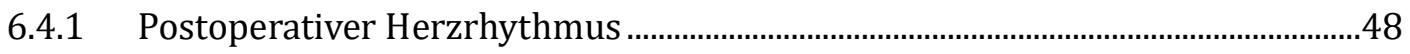

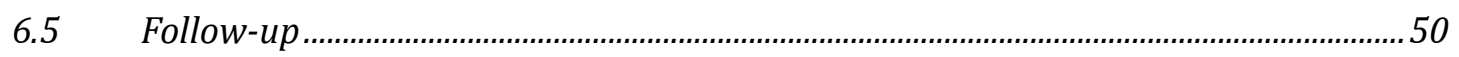

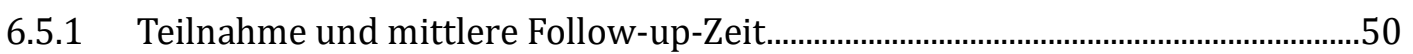

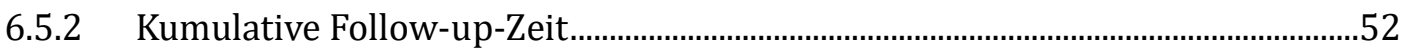

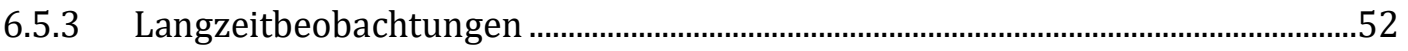

6.5.4 Herzrhythmus im letzten Follow-up (inkl. Grundrhythmus bei Schrittmacher) 52

6.5.5 Schrittmacheranzahl im letzten Follow-up ..............................................................56

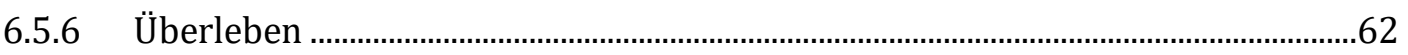

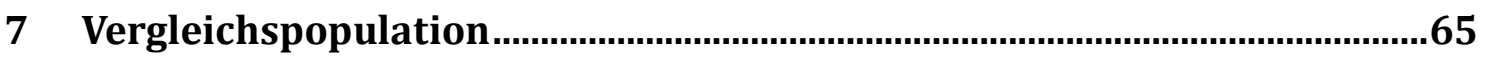

7.1 Überleben von Gruppe A versus Vergleichspopulation...................................................65

7.2 Überleben von Gruppe B versus Vergleichspopulation...................................................66

7.3 Überleben von Gruppe C versus Vergleichspopulation...............................................66

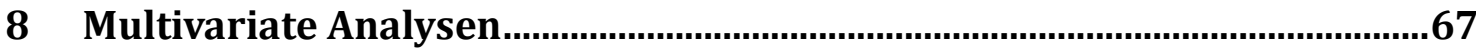


8.1 Logistische Regression: Ablationserfolg bei Entlassung und im letzten Follow-up 67

8.1.1 Logistische Regression: Ablationserfolg bei Entlassung .........................................68

8.1.2 Logistische Regression: Ablationserfolg im Last Follow-up....................................68

8.2 Cox-Regression: Einfluss auf die Schrittmacherhäufigkeit...........................................69

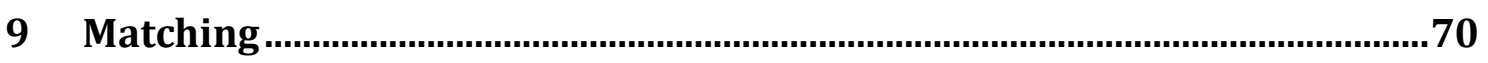

$9.1 \quad$ Methoden

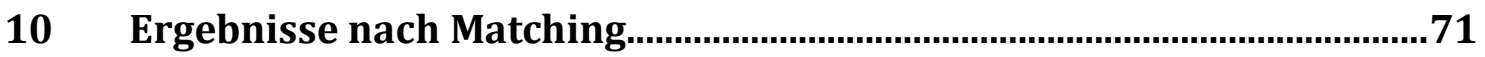

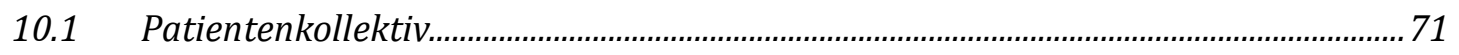

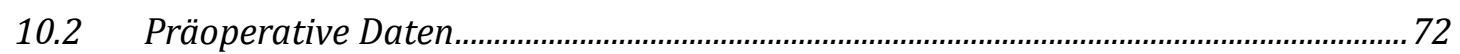

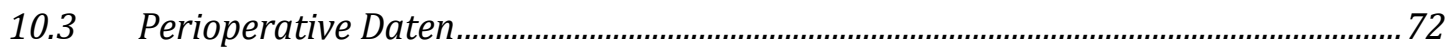

10.3.1 Perioperative Schrittmacherimplantationen ...........................................................73

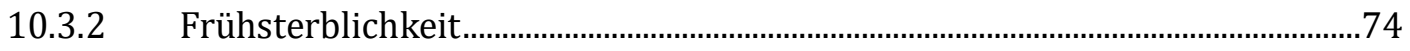

$10.4 \quad$ Postoperative Daten (= zum Zeitpunkt der Entlassung) ............................................. 74

10.4.1 Postoperativer Herzrhythmus ............................................................................

10.4.2 Postoperative Schrittmacheranzahl.....................................................................76

$10.5 \quad$ Follow-up

10.5.1 Teilnahme und mittlere Follow-up-Zeit ………....................................................77

10.5.2 Kumulative Follow-up-Zeit …………...................................................................

10.5.3 Langzeitbeobachtungen ..................................................................................

10.5.4 Herzrhythmus im letzten Follow-up (inkl. Grundrhythmus bei

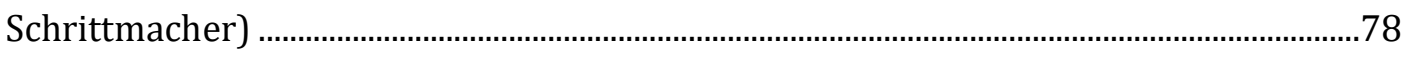

10.5.5 Schrittmacher im letzten Follow-up ................................................................... 82

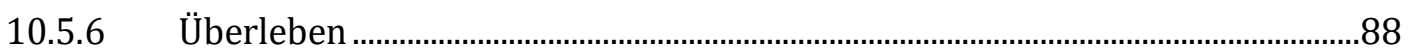

11 Multivariate Analysen nach Matching ............................................................

11.1 Logistische Regression: Ablationserfolg bei Entlassung und im letzten Follow-up 91 
11.2 Logistische Regression: Ablationserfolg bei Entlassung............................................... 92

11.3 Logistische Regression: Ablationserfolg im Last Follow-up ......................................... 92

11.4 Cox-Regression: Einfluss auf die Schrittmacherhäufigkeit..........................................93

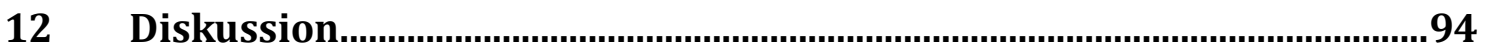

12.1 Inzidenz von Vorhofflimmern bei präoperativem Sinusrhythmus...............................95

12.2 Inzidenz von Schrittmacherimplantationen ...............................................................97

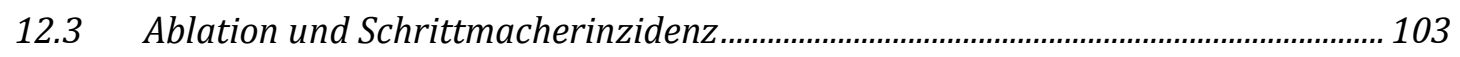

12.4 Ablationserfolg bei präoperativ bestehendem Vorhofflimmern ................................. 104

$12.5 \quad$ Überleben

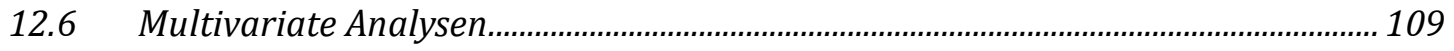

12.7 Untersuchung verschiedener Merkmale auf die Schrittmacherhäufigkeit .............110

12.8 Untersuchung verschiedener Merkmale auf den Ablationserfolg............................. 111

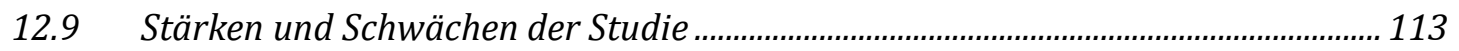

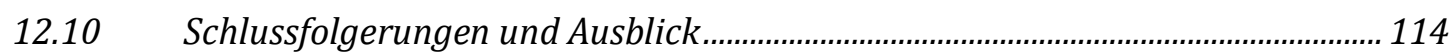

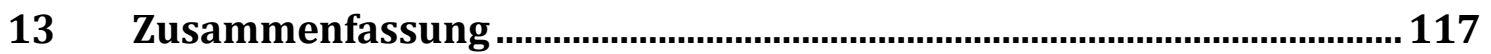

14 Summary

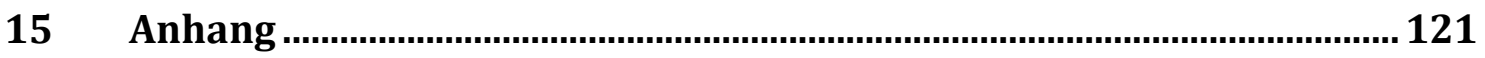

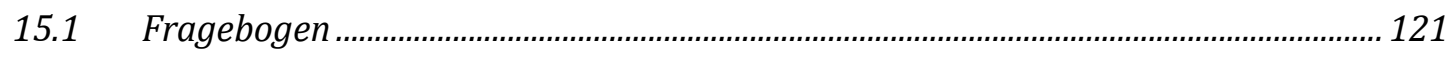

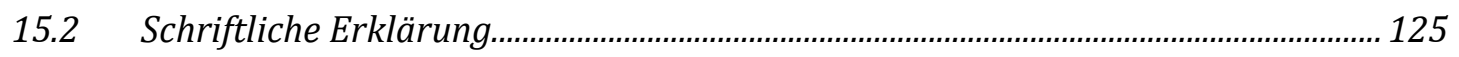

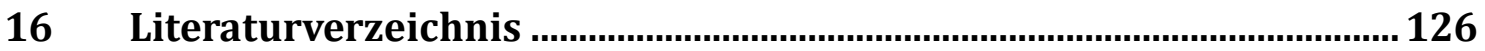




\section{Abkürzungsverzeichnis, Abbildungsverzeichnis, Tabellenverzeichnis}

\subsection{Abkürzungsverzeichnis}

ACB

AKE

ASD

bspw.

d. h.

EKG

FU

ICR

inkl.

$\mathrm{KHK}$

LA

LVEF

LVESD

MI

MKE

MKR

MÖV

MS

NOAK

o. $g$.

$\mathrm{OP}$

OR

PDA

PFO
Arteriocoronarer Bypass

Aortenklappenersatz

Atriumseptumdefekt

beispielsweise

das heißt

Elektrokardiogramm

Follow-up

Intercostalraum

inklusive

koronare Herzkrankheit

linkes Atrium

linksventrikuläre Funktion

linksventrikulärer enddiastolischer Diameter

Mitralklappeninsuffizienz

Mitralklappenersatz

Mitralklappenrekonstruktion

Mitralklappenöffnungsfläche

Mitralklappenstenose

neue orale Antikoagulantien

oben genannt

Operation

Odds Ratio

persistierender Ductus arteriosus

persistierendes Foramen ovale 
PMC

POS

PTCA

SD

SM

SR

TEE

THG-Ambulanz

TKR

TTE

v. a.

VHF

VS.

z. B.
Mitralklappencomissurotomie

partielle obere Sternotomie

perkutane transluminale Koronarangioplastie

Standardabweichung

Schrittmacher

Sinusrhythmus

transösophageale Echokardiographie

Thorax-, Herz- und thorakale Gefäßchirurgie-Ambulanz

Trikuspidalklappenrekonstruktion

transthorakale Echokardiografie

vor allem

Vorhofflimmern

versus

zum Beispiel 


\subsection{Abbildungsverzeichnis}

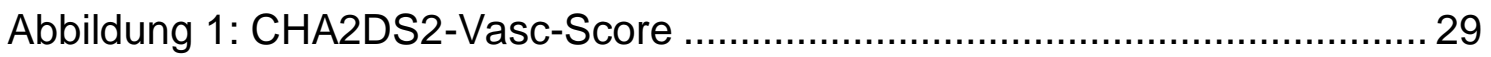

Abbildung 2: Häufigkeit der Zugangswege im Patientenkollektiv ..................... 40

Abbildung 3: Vorhofflimmeranteil der Vergleichsgruppen im Verlauf .................55

Abbildung 4: Vorhofflimmeranteil der Vergleichsgruppen nach Ablation im

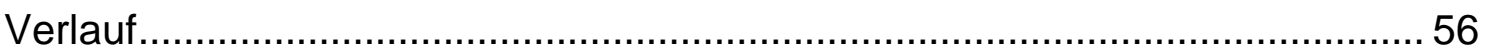

Abbildung 5: Schrittmacheranteil der Vergleichsgruppen im Verlauf ................ 58

Abbildung 6: Kaplan-Meier-Kurve: Freiheit von Schrittmacherimplantationen.. 60

Abbildung 7: Kaplan-Meier-Kurve: Freiheit von Defibrillatorimplantationen .......61

Abbildung 8: Kaplan-Meier-Kurve: Überleben ................................................. 63

Abbildung 9: Überleben Gruppe A vs. Vergleichspopulation............................. 65

Abbildung 10: Überleben Gruppe B vs. Vergleichspopulation ........................... 66

Abbildung 11: Überleben Gruppe C vs. Vergleichspopulation........................... 66

Abbildung 12: Vorhoffimmeranteil der Vergeichsgruppe (nach Matching) im

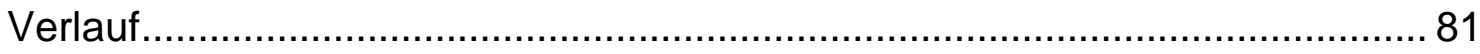

Abbildung 13: Vorhofflimmeranteil der Vergleichsgruppen nach Ablation (nach

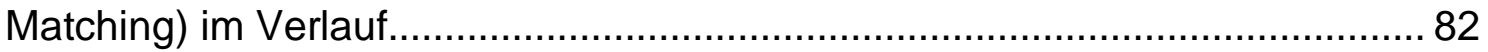

Abbildung 14: Schrittmacheranteil der Vergleichsgruppen (nach Matching) im Verlauf

Abbildung 15: Kaplan-Meier-Kurve: Freiheit von Schrittmacherimplantationen (nach Matching)

Abbildung 16: Kaplan-Meier-Kurve: Freiheit von Defibrillatorimplantationen (nach Matching)

Abbildung 17: : Kaplan-Meier-Kurve: Überleben (nach Matching) 89 


\subsection{Tabellenverzeichnis}

Tabelle 1: Geschlecht und Alter der Studienpopulation ................................... 41

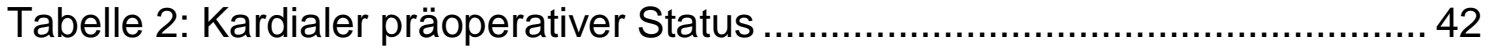

Tabelle 3: Präoperativer Herzrhythmus ........................................................ 42

Tabelle 4: Patienten mit präoperativem Herzschrittmacher .............................. 43

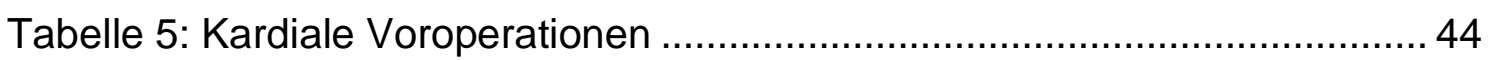

Tabelle 6: Mitralklappeneingriff und zusätzliche Eingriffe ............................... 45

Tabelle 7: Perioperativ implantierte Schrittmacheranzahl............................... 46

Tabelle 8: Perioperativ implantierte Schrittmacheranzahl nach Ablation .......... 46

Tabelle 9: Perioperativ implantierte Schrittmacheranzahl der nicht abladierten

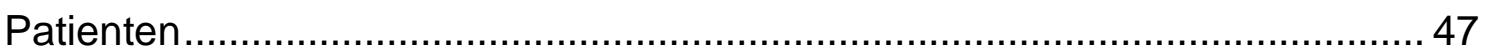

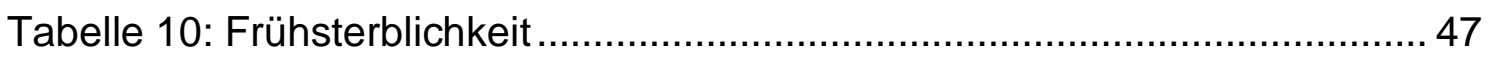

Tabelle 11: Postoperativer Herzrhythmus bei Entlassung ................................ 48

Tabelle 12: Postoperativer Herzrhythmus nach Ablation bei Entlassung.......... 49

Tabelle 13: Schrittmacheranzahl bei Entlassung ………………….............. 49

Tabelle 14: Postoperativer Herzrhythmus der nicht abladierten Patienten bei

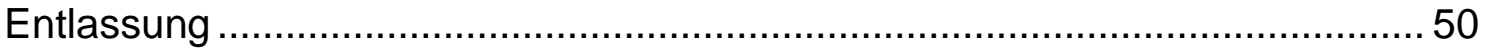

Tabelle 15: Postoperativer Herzrhythmus der Patienten mit präoperativem

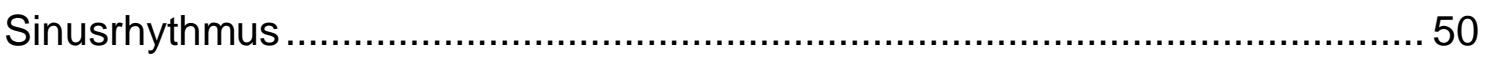

Tabelle 16: Follow-up-Anzahl unter den Überlebenden.................................... 51

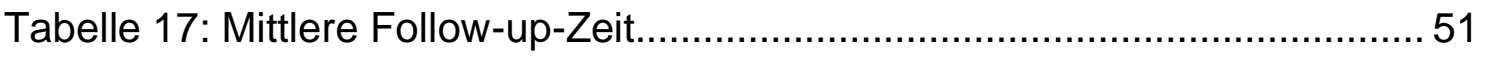

Tabelle 18: Herzrhythmus im letzten Follow-up ........................................... 52

Tabelle 19: Herzrhythmus im letzten Follow-up nach Ablation............................ 53

Tabelle 20: Herzrhythmus der nicht abladierten Patienten im letzten Follow-up

Tabelle 21: Herzrhythmus der Patienten mit präoperativem Sinusrhythmus zum Zeitpunkt des letzten Follow-ups 54

Tabelle 22: Absolute Schrittmacherzahl zum Zeitpunkt des letzten Follow-ups56 Tabelle 23: Ereignisanzahl Schrittmacher- und Defibrillatorimplantationen ...... 59 Tabelle 24: Kumulative Schrittmacherfreiheit 60 
Tabelle 25: Kumulative Defibrillatorfreiheit

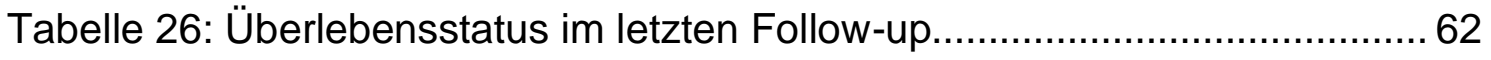

Tabelle 27: Kumulierter Anteil Überlebender.

Tabelle 28: Logistische Regression: Einflussfaktoren auf den Ablationserfolg bei

Entlassung 68

Tabelle 29: : Logistische Regression: Einflussfaktoren auf den Ablationserfolg im letzten Follow-up 68

Tabelle 30: Cox-Regression: Einfluss auf die Schrittmacherhäufigkeit 69

Tabelle 31: Zielvariablen des Matchings 71

Tabelle 32: Präoperativer Herzrhythmus (nach Matching) 72

Tabelle 33: Patienten mit präoperativem Herzschrittmacher (nach Matching) . 72

Tabelle 34: Perioperativ abladierte Patienten (nach Matching 72

Tabelle 35: Anzahl perioperativ implantierter Schrittmacher (nach Matching).. 73 Tabelle 36: Anzahl perioperativ implantierter Schrittmacher nach Ablation (nach Matching) 73

Tabelle 37: Anzahl perioperativ implantierter Schrittmacher der nicht abladierten

Patienten (nach Matching) 73

Tabelle 38: Frühsterblichkeit (nach Matching)......................................... 74

Tabelle 39: Postoperativer Herzrhythmus bei Entlassung (nach Matching)...... 75

Tabelle 40: Postoperativer Herzrhythmus nach Ablation bei Entlassung (nach

Matching)

Tabelle 41: Postoperativer Herzrhythmus der nicht Abladierten bei Entlassung (nach Matching)

Tabelle 42: Postoperativer Herzrhythmus der Patienten mit präoperativem

Sinusrhythmus (nach Matching) 76

Tabelle 43: Schrittmacheranzahl bei Entlassung (nach Matching) ................... 76

Tabelle 44: Follow-up-Anzahl unter den Überlebenden (nach Matching) ......... 77

Tabelle 45: Mittlere Follow-up-Zeit (nach Matching) ....................................... 77

Tabelle 46:Herzrhythmus im letzten Follow-up (nach Matching) ...................... 78 
Tabelle 47: Herzrhythmus im letzten Follow-up nach Ablation (nach Matching)

Tabelle 48: Herzrhythmus im letzten Follow-up der nicht Abladierten (nach

Matching)

Tabelle 49: Herzrhythmus der Patienten mit präoperativem Sinusrhythmus zum

Zeitpunkt des letzten Follow-ups (nach Matching) 79

Tabelle 50: Absolute Schrittmacherzahl zum Zeitpunkt des letzten Follow-ups (nach Matching)

Tabelle 51: Ereignisanzahl Schrittmacher- und Defibrillatorimplantationen (nach Matching) 85

Tabelle 52: Kumulative Schrittmacherfreiheit (nach Matching) 86

Tabelle 53: Kumulative Defibrillatorfreiheit (nach Matching) 88

Tabelle 54: Überlebensstatus im letzten Follow-up (nach Matching) 88

Tabelle 55: Kumulierter Anteil Überlebender (nach Matching) 90

Tabelle 56: Logistische Regression: Einflussfaktoren auf den Ablationserfolg bei Entlassung (nach Matching). 92

Tabelle 57: Logistische Regression: Einflussfaktoren auf den Ablationserfolg im letzten Follow-up (nach Matching) 92

Tabelle 58: Cox-Regression: Einfluss auf die Schrittmacherhäufigkeit (nach Matching) 93 


\section{Einleitung}

Die vorliegende Dissertation beschäftigt sich mit der Veränderung des postoperativen Herzrhythmus, insbesondere dem Auftreten von Vorhofflimmern sowie der Notwendigkeit einer Schrittmacherimplantation nach minimalinvasiver Mitralklappenchirurgie in Abhängigkeit vom Zugangsweg zur Mitralklappe. Zur Einführung werden die wesentlichen Aspekte des zugrundeliegenden Mitralklappendefektes sowie das Krankheitsbild des Vorhofflimmerns und die insbesondere chirurgischen Therapiemethoden geschildert.

\subsection{Mitralklappenvitien}

Unter dem Begriff Mitralklappenvitien werden Funktionsstörungen der Mitralklappe zusammengefasst.

Betrifft der Defekt die Klappenöffnung, spricht man von einer Mitralklappenstenose. Bei der Mitralklappeninsuffizienz hingegen ist die Schließfunktion der Mitralklappe vermindert. Die Defekte können sowohl isoliert als auch in Kombination auftreten.

\subsubsection{Anatomie der Mitralklappe}

Die Mitralklappe befindet sich im linken Herzen zwischen dem linken Atrium und dem linken Ventrikel. Sie besitzt die Funktion eines Ventils und ermöglicht damit das Fließen des Blutes aus dem Vorhof in die Kammer (und nicht umgekehrt). Sie besteht aus dem Mitralklappenanulus, an dem zwei Segeln ansetzen. Diese sind wie ein geteilter Fallschirm in den Ventrikel verspannt und dort am subvalvulären Apparat (Papillarmuskeln und Chordafäden) befestigt. Während der Systole ist das Ventil geschlossen, die freien Ränder der Segel werden durch die Blutströmung aneinandergelegt, wobei die Sehnenfäden ein komplettes Umschlagen der Segel in den Vorhof verhindern. Während der Diastole ist das Ventil geöffnet, so dass Blut aus dem Vorhof durch einen 
trichterförmigen Kanal in den Ventrikel fließen kann. Durch die Herzaktion ändert sich auch die Form und Größe des beweglichen Mitralanulus. In der Systole ist dieser kontrahiert und erleichtert so die Koaptation der Mitralsegel, während er in der Diastole erweitert ist. ${ }^{1}$

\subsubsection{Mitralklappeninsuffizienz}

\subsubsection{Epidemiologie}

Die Mitralklappeninsuffizienz (MI) ist beim Erwachsenen das zweithäufigste behandlungsbedürftige Herzklappenvitium nach der Aortenklappenstenose. Die Prävalenz der Mitralklappeninsuffizienz steigt mit zunehmendem Lebensalter an. ${ }^{2,3}$

\subsubsection{2 Ätiologie}

Die Mitralklappeninsuffizienz kann in eine primäre und eine sekundäre Form unterteilt werden.

Bei der primären bzw. degenerativen Form handelt es sich um meist erworbene Pathologien an der Mitralklappe oder ihrem Halteapparat.

Ursächlich hierfür können sein:

- Mitralklappenprolaps

- degenerative Veränderung von Mitralklappenring oder Mitralklappensegeln (myxomatöse Degeneration der Klappenstruktur)

- Barlow-Erkrankung (Vermehrung der Klappenstruktur)

- rheumatische oder bakterielle Endokarditiden

- angeborene Herzfehler (z.B. partieller AV-Kanal)

- Marfan-Syndrom, Ehlers-Danlos-Syndrom

Die sekundäre bzw. funktionelle Mitralklappeninsuffizienz entsteht als Folgeerkrankung von pathologischen Umbauprozessen des linken Herzens wie beispielsweise einer ausgeprägten Dilatation des linken Ventrikels oder einer eingeschränkten linksventrikulären Funktion. 
Diese Prozesse resultieren in einer Dilatation des Mitralklappenanulus und/oder im Verlust der Adaptation der Mitralsegel und damit des suffizienten

Klappenschlusses. ${ }^{4,5}$

Ursächlich hierfür können sein:

- Kardiomyopathie

- Myokarditis

- Myokardinfarkt (ischämisch)

- Vorhofflimmern

\subsubsection{Pathophysiologie}

Eine Mitralklappeninsuffizienz kann akut oder chronisch auftreten und die oben geschilderten primären und sekundären Ursachen haben.

Pathophysiologisch kommt es unabhängig von der zugrunde liegenden Ursache zu einem Defekt des Mitralklappenschlusses zwischen dem linken Atrium und dem linken Ventrikel. Dies hat zur Folge, dass während der Systole das Blutvolumen des linken Ventrikels nicht nur in den Systemkreislauf ausgeworfen wird, sondern auch zurück in das linke Atrium gelangt. Durch diese Volumenbelastung kommt es zu einer Dilatation des linken Herzens, welche die Mitralklappeninsuffizienz wiederum selbst verstärkt. Des Weiteren kann das Regurgitationsvolumen aus dem linken Atrium zurück in die Pulmonalvene gelangen, zu einer pulmonalen Hypertonie führen und wiederum das rechte Herz und seine Klappen belasten, mit der Folge einer sekundären Trikuspidalklappeninsuffizienz.

Die akute Form kann insbesondere nach Myokardinfarkt und bakterieller Endokarditis auftreten oder auch infolge eines Traumas mit Herzklappenbeteiligung.

Schlussendlich kann eine fortgeschrittene Mitralklappeninsuffizienz zur Herzinsuffizienz mit kardialer Dekompensation und Lungenödem führen. Des Weiteren ist das Risiko für eine Endokarditis bei Herzklappenvitien erhöht. 
Durch eine erhöhte Prävalenz von Vorhofflimmern in Assoziation mit einer ausgeprägten Dilatation des linken Vorhofs ist das Risiko für Thromboembolien erhöht. ${ }^{4-6}$

\subsubsection{Diagnostik}

Hinweise auf eine Mitralklappeninsuffizienz geben klinische Symptome einer Herzinsuffizienz wie Belastungsdyspnoe und Ödeme. Diese treten jedoch meist erst bei fortgeschrittener Mitralklappeninsuffizienz auf. Im Elektrokardiogramm (EKG) kann ein Vorhofflimmern hinweisend sein. Weiterführend ist die transthorakale Echokardiografie (TTE), mit der die Schweregradeinteilung der $\mathrm{Ml}$ erfolgen sowie auch die zugrundeliegende Pathologie identifiziert werden kann. Des Weiteren können hier die linksventrikuläre Funktion (LVEF) sowie die Diameter der Herzhöhlen ermittelt werden. Auch die Quantifizierung eines pulmonalen Hypertonus mittels Flussmessung über der Trikuspidalklappe und die Beurteilung der Rechtsherzbelastung liefern wichtige Bausteine für die Schweregradeinteilung der MI. Diese erfolgt in die 3 Schweregrade leicht-, mittel- und hochgradig.

Zur ergänzenden Beurteilung sollte eine transösophageale Echokardiographie (TEE) erfolgen, die detaillierte Aussagen hinsichtlich Morphologie und Rekonstruierbarkeit der Mitralklappe gibt.

Die Herzkatheteruntersuchung ist insbesondere bei Patienten $>45$ Jahre indiziert, um eine begleitende koronare Herzkrankheit (KHK) zu evaluieren (Linksherzkatheter). Des Weiteren dient sie zur Messung der pulmonalen Hypertonie, des pulmonalen Widerstandes und der linksventrikulären Pumpfunktion (Rechtsherzkatheter). ${ }^{4,7}$

\subsubsection{Therapie}

Aufgrund der oben geschilderten pathophysiologischen Mechanismen sollte man eine Therapie der Mitralklappeninsuffizienz möglichst frühzeitig angehen, um einer Herzinsuffizienz vorzubeugen. 
Die konservative Therapie beschränkt sich auf rein symptomatische Therapie mittels körperlicher Schonung und optimaler medikamentöser Herzinsuffizienztherapie sowie Antikoagulation und Frequenzkontrolle bei begleitend vorliegendem Vorhofflimmern. ${ }^{4}$

Die chirurgische Therapie ist die einzige kausale Therapie ${ }^{8}$ und der Goldstandard für Patienten mit hochgradiger primärer Mitralklappeninsuffizienz. Zu bevorzugen ist hierbei die Rekonstruktion der Mitralklappe, die im Vergleich zum Klappenersatz eine wesentlich geringere perioperative Mortalität aufweist. Sie stellt eine nachhaltig kurative Therapie dar ${ }^{9}$, die in den Langzeitergebnissen dem Klappenersatz überlegen ist. ${ }^{10}$ Des Weiteren kann auf die beim mechanischen Mitralklappenersatz (MKE) notwendige Antikoagulation verzichtet werden, ${ }^{4,8}$ Ein biologischer Klappenersatz, der keiner Antikoagulation bedarf, weist das Problem der begrenzten Haltbarkeit auf. Ist eine Rekonstruktion nicht möglich, wie z. B. bei funktioneller Mitralklappeninsuffizienz sowie bei starken Verkalkungen oder entzündlich veränderter Klappenstruktur, muss ein Klappenersatz erfolgen. Hier wird der biologische Klappenersatz mit Erhalt des subvalvulären Apparates allein schon aufgrund der eingeschränkten Lebenserwartung bevorzugt.

Bei einer dilatativen Kardiomyopathie sind die Langzeitergebnisse beim Klappenersatz denen der Rekonstruktion überlegen ${ }^{11}$, insbesondere bei deutlicher Ventrikeldilatation mit konsekutivem Tenting.

Die Rezidive bei der Mitralklappenrekonstruktion (MKR) sind auf verschiedene Faktoren zurückzuführen (z. B. angewandte Technik, Erholung der linksventrikulären Funktion, Verschiebung der Koaptationslinie). Bei Patienten, die durch Komorbiditäten ein zu hohes Operationsrisiko aufweisen oder inoperabel sind, sowie bei schwerer Kardiomyopathie und hochgradig eingeschränkter linksventrikulärer Funktion, sind kathetergestützte Verfahren wie der Mitra-Clip derzeit das Mittel der Wahl. ${ }^{12}$ 


\subsection{Indikation zur Mitralklappenchirurgie}

Die Indikation zur Operation richtet sich nach Ätiologie, Schweregrad der MI und Symptomatik des Patienten.

Bei einer akut aufgetretenen MI besteht grundsätzlich eine Indikation zur raschen operativen Therapie mittels Mitralklappenrekonstruktion oder Mitralklappenersatz. ${ }^{4}$

Bei der chronischen Form wird je nach Ätiologie unterschieden.

\section{Primäre bzw. degenerative MI}

Asymptomatische Patienten mit chronischer geringer und mittelgradiger MI, bei denen noch keine OP-Indikation besteht, sollte man in regelmäßigen Abständen echokardiografisch untersuchen (watchfull waiting) ${ }^{13}$, um eine Progredienz rechtzeitig zu detektieren und Spätfolgen vorzubeugen.

Bei chronischer hochgradiger $\mathrm{Ml}$ ist die Operationsindikation abhängig von der Symptomatik des Patienten.

Asymptomatische Patienten sollten operiert werden, sobald die linksventrikuläre Ejektionsfraktion eingeschränkt (LVEF < 60 \%) und/oder der linksventrikuläre enddiastolische Diameter vergrößert ist (LVESD > $45 \mathrm{~mm}$ ). Besteht eine normale LVEF, jedoch ein valvuläres Vorhofflimmern oder eine pulmonale Hypertonie (50 mmHg), ist eine OP ebenfalls empfohlen. ${ }^{4,14}$

Nach aktueller Richtlinie der European Association for Cardio-Thoracic Surgery (EACTS) und der European Society of Cardiology (ESC) von 2017 wurde die Indikation zur Operation für asymptomatische Patienten mit hochgradiger isolierter Ml aufgrund einer erhöhten Mortalität beim konservativen Weg erweitert. ${ }^{15}$ Demzufolge besteht eine Operationsindikation für asymptomatische Patienten mit isolierter hochgradiger primärer MI aufgrund von Segelprolaps (flail leatflet), wenn die Operation in einem Expertenzentrum mit geringer Mortalität durchgeführt wird. ${ }^{14}$

Bei symptomatischen Patienten besteht stets eine Indikation zur operativen Versorgung, sofern eine LVEF > 30 \% gegeben ist. 
Bei einer LVEF < $30 \%$ und ausgeschöpfter medikamentöser Therapie ist bei gegebener Rekonstruktionsfähigkeit der Mitralklappe eine Operation ebenfalls indiziert. ${ }^{4}, 14$

Bei Patienten mit zu hohem Operationsrisiko aufgrund hohen Alters und Komorbiditäten und/oder einer LVEF $<30 \%$ kann nach Evaluation im Heart Team eine MitraClip-Versorgung erfolgen. ${ }^{12}$

\section{Sekundäre, funktionelle MI}

Grundsätzlich wird die sekundäre Ml bedingt und begleitet durch eine eingeschränkte LVEF und/oder eine linksventrikuläre Dilatation. Aufgrund dessen und wegen der in dieser Patientengruppe meist zusätzlich bestehenden Komorbiditäten ist das Operationsrisiko wesentlich höher als bei der primären Ml.

Daher steht hier zunächst eine optimale Herzinsuffizienztherapie im Fokus. Des Weiteren kann es nach Resynchronisationstherapie zu einer Verbesserung der Ml kommen. ${ }^{16}$

Besteht bei diesen Patienten bezüglich einer zugrundeliegenden KHK eine Bypassindikation, kann eine gleichzeitig durchgeführte Mitralklappenrekonstruktion das Überleben verbessern. ${ }^{17}$ Die Indikation ist jedoch, insbesondere bei stark eingeschränkter linksventrikulärer Pumpfunktion, noch nicht klar definiert und muss im Einzelfall diskutiert werden.

Die Indikation zur operativen Versorgung kann also bei sekundärer MI bestehen, wenn gleichzeitig eine Indikation zur operativen Myokardrevaskularisation vorliegt. Bei Patienten ohne Revaskularisierungsbedarf und mit hochgradiger MI (trotz optimaler medikamentöser Therapie) muss bezüglich der OP-Indikation eine interdisziplinäre Nutzen-Risiko-Abwägung unter Berücksichtigung der Komorbiditäten erfolgen. Bei Hochrisikopatienten sollte eine MitraClip-Indikation evaluiert werden. 


\subsubsection{Mitralklappenstenose}

\subsubsection{Epidemiologie}

Die Mitralklappenstenose (MS) ist weltweit einer der häufigsten erworbenen Herzklappenfehler. In der westlichen Welt ist sie aufgrund des Einsatzes von Antibiotika bei Streptokokkeninfektionen seltener geworden. Insgesamt sind Frauen häufiger betroffen als Männer.4, 18, 19 Häufig zeigt sich ein kombiniertes Vitium aus Mitralklappenstenose und Aortenklappenstenose. ${ }^{20}$

\subsubsection{2 Ätiologie}

Die weltweit häufigste Ursache für die Mitralklappenstenose ist das rheumatische Fieber und die damit verbundene Endokarditis. In der westlichen Welt ist die degenerativ verkalkende Form die häufigste.

Sehr selten ist eine kongenitale Fehlbildung oder infektiös bedingte Endokarditis ursächlich. ${ }^{4,18,19}$

\subsubsection{Pathophysiologie}

Die Mitralklappenstenose entsteht schleichend über Jahre aufgrund von degenerativen Veränderungen der Klappenstrukturen. Durch Fibrosierung und Verkalkung kommt es zu einer zunehmend verminderten Öffnungsfläche der Mitralklappe. ${ }^{20}$ Dies führt zu einer verminderten linksventrikulären Füllung und damit zu vermindertem Auswurfvolumen in den Systemkreislauf. Weiterhin kommt es zu einer erhöhten Druck- und Volumenbelastung des linken Vorhofs mit daraus resultierender Dilatation und häufigem Auftreten von Vorhofflimmern. Durch Rückstau in die pulmonalen Gefäße kommt es zur konsekutiven pulmonalen Hypertonie und Rechtsherzbelastung. ${ }^{4,6}$ 


\subsubsection{Diagnostik}

Klinisch zeigen sich Symptome der Herzinsuffizienz sowie in vielen Fällen Vorhofflimmern mit Palpitationen. Häufig wird die Diagnose erst nach einer durch das Vorhofflimmern hervorgerufenen Thromboembolie gestellt. ${ }^{6}$ Wegweisend können klinische Untersuchung, Röntgen-Thorax und EKG sein, wobei die entscheidende Bestimmung der Klappenöffnungsfläche, des transmitralen Druckgradienten sowie des Ausmaßes des pulmonalen Hypertonus schließlich mittels Echokardiografie erfolgt. Anhand dieser Parameter erfolgt auch die Schweregradeinteilung der MS nach Mitralklappenöffnungsfläche (MÖV). ${ }^{6}$

\subsubsection{Therapie}

Die Therapiemöglichkeiten sind ähnlich denen bei der Mitralklappeninsuffizienz:

- konservative Therapie

- kathetergestützte Verfahren

- chirurgischer Mitralklappenersatz

Die konservative Therapie entspricht der medikamentösen Herzinsuffizienztherapie sowie einer Thromboembolieprophylaxe bei Vorhofflimmern. Des Weiteren sollte eine Endokarditisprophylaxe erfolgen. Grundsätzlich besteht die Indikation zur Intervention ab einer mittelschweren Mitralklapppenstenose (Mitralklappenöffnungsfläche von $<1,5 \mathrm{~cm}^{2}$ ); sie ist abhängig von der Symptomatik des Patienten.

Kathetergestützt kann eine perkutane Mitralklappencomissurotomie (PMC, Mitralvalvuloplastie) erfolgen, bei der die verschmolzenen Kommissuren der Mitralklappe mittels Ballon aufgesprengt werden.

Diese Methode ist aufgrund ihres minimalinvasiven Charakters das Mittel der Wahl, hat aber strenge Selektionskriterien in Bezug auf die Morphologie der Klappe. 
In den folgenden Fällen ist jedoch eine chirurgische Therapie mittels Mitralklappenersatz indiziert:4, 14

- erheblich symptomatische Patienten und MÖV $>1,5 \mathrm{~cm}^{2}$

- gering oder nicht symptomatische Patienten mit MÖV $<1 \mathrm{~cm}^{2}$

- zusätzliche Operationsindikationen wie Bypassindikation oder andere operationswürdige Klappenvitien.

\subsubsection{Minimalinvasive Mitralklappenchirurgie}

Grundsätzlich ist die Operation der Mitralklappe eine der häufigsten Herzklappenoperationen.

Minimalinvasive Zugangswege in der Chirurgie haben im Allgemeinen in den vergangenen Jahren stark an Bedeutung gewonnen. Sie bringen durch kleinere Schnitte einen geringeren Blutverlust, ein geringeres Risiko für Wundheilungsstörungen und Infektionen sowie ein besseres kosmetisches Ergebnis mit höherer Patientenzufriedenheit mit sich.

Bis Mitte der 1990er-Jahre stellte die mediane Sternotomie den Standardzugangsweg zur Mitral- und Aortenklappe dar. Die Komplikationsrate war zwar verhältnismäßig gering, jedoch die Mortalität bei Auftreten von Sternumdehiszenz, Osteomyelitis und Mediastinitis hoch. ${ }^{21-23}$ Daher wurde die Entwicklung minimalinvasiver Zugangswege insbesondere in den vergangenen zwei Jahrzehnten in diesem Bereich stark vorangetrieben. Ergebnisse waren die partielle Sternotomie und die anterolaterale Minithorakotomie, bei deren Verwendung auf eine vollständige Eröffnung des Sternums verzichtet werden kann. ${ }^{24}$ Die minimalinvasiven Zugangswege verringern das Risiko für respiratorische Komplikationen, ermöglichen eine schnellere Mobilisierung und erhöhen die Patientenzufriedenheit durch bessere kosmetische Ergebnisse sowie schnellere Erholung und geringere Schmerzbelastung. Des Weiteren konnten trotz technisch erhöhtem Aufwand geringere Kosten durch kürzere intensivstationäre Aufenthalte sowie insgesamt kürzere 
Krankenhausaufenthalte verzeichnet werden.$^{25} \mathrm{Im}$ Hinblick auf das Überleben zeigte sich im Vergleich zur medianen Sternotomie kein Unterschied. ${ }^{26}$

Dennoch ist die Evidenz laut einem Review von 2017 gering. ${ }^{27}$ Die minimalinvasiven Eingriffe sind technisch hoch anspruchsvoll und bedürfen der Durchführung an einem Zentrum mit hohem Patientenaufkommen und großer Erfahrung.

Die meisten herzchirurgischen Eingriffe werden daher heutzutage weiterhin über eine komplette mediane Sternotomie durchgeführt.

Am häufigsten kommen minimalinvasive Zugangswege in der Herzchirurgie bei Mitralklappenoperationen zum Einsatz.

Im Wesentlichen gibt es drei mögliche Zugangswege zur Mitralklappe, die abhängig von Komorbiditäten und vom Umfang des Eingriffs gewählt werden:

- mediane Sternotomie

- anterolaterale Minithorakotomie

- partielle obere Sternotomie

Die mediane Sternotomie liefert aufgrund des freien Zugangs zum ganzen Herzen die Möglichkeit von Kombinationseingriffen - insbesondere kombinierte Bypass- und Klappenchirurgie.

Die beiden letzteren, minimalinvasiven Zugangswege sind Untersuchungsgegenstand dieser Arbeit und werden in Folgenden genauer erläutert.

2.1.4.1 Anterolaterale Minithorakotomie unter Verwendung der ChitwoodKlemme

Die erste videoassistierte anterolaterale Minithorakotomie wurde von A. Carpentier 1996 durchgeführt. ${ }^{24}$ Prof. Chitwood führte kurz darauf eine neue, für diese Operationsmethode verwendbare transthorakale Aortenklemmung ein - die Chitwood-Klemme. ${ }^{28}$ 


\section{OP-Vorbereitung und Durchführung}

Der Patient liegt in Rückenlage mit leicht angehobenem rechtsseitigem Hemithorax. Die Intubation erfolgt mittels Doppellumentubus, um eine Einlungenventilation zu ermöglichen. Der Anschluss an die Herz-LungenMaschine erfolgt über die Femoralgefäße.

Die anterolaterale Minithorakotomie wird rechtsseitig im 4. Interkostalraum (ICR) inframamillär mit einem 4 bis $6 \mathrm{~cm}$ langen Hautschnitt durchgeführt. Unter Diskonnektion der rechten Lunge und Einlungenventilation über die linke Lunge wird das Perikard unter Schonung des N. phrenicus eröffnet. Die Kanülierung zur Herz-Lungen-Maschine erfolgt im Allgemeinen über die Leistengefäße. Die transthorakale Aortenklemmung mittels Chitwood-Klemme erfolgt im 2. ICR rechts in der hinteren Axillarlinie. ${ }^{28}$ Durch Gabe einer kardioplegen Lösung in die Aortenwurzel (antegrade Kardioplegie) kommt es zur Asystolie. Über einen zusätzlichen Kameraport kann der Eingriff mithilfe eines Bildschirms erfolgen: meist ist dem Chirurgen jedoch eine direkte Visualisierung über die Minithorakotomie möglich. Des Weiteren wird über den Kameraport kontinuierlich Kohlendioxid insuffliert, um Luftembolien zu vermeiden. ${ }^{29}$ Zur optimalen Vorhofvisualisierung erfolgt nun eine weitere Inzision rechts parasternal und die Einbringung des Vorhofretraktors in den linken Vorhof nach Linksatriotomie. Nun kann die Rekonstruktion bzw. der Ersatz der Mitralklappe erfolgen. Zusätzlich kann eine Mitversorgung der Trikuspidalklappe, ein ASDVerschluss, eine modifizierte Maze-Prozedur oder ein Vorhofohrverschluss bei Vorhofflimmern erfolgen. ${ }^{30}$

Kontraindikationen für die anterolaterale Minithorakotomie sind:

- Voroperationen und Verwachsungen im Bereich des rechten Hemithorax, z. B. bei Zweiteingriffen

- ausgeprägte Mitralringverkalkung bis in das tiefe Myokard reichend

- hochgradige Sklerose der Aorta ascendens,

- zusätzliche Klappenerkrankungen,

- KHK mit Bypassindikation 


\subsubsection{Partielle obere Sternotomie}

Die partielle obere Sternotomie (POS) wurde erstmals 1997 zur Anwendung bei Mitralklappenersatz und -rekonstruktion beschrieben. ${ }^{31}$

OP-Vorbereitung und Durchführung

Der Patient liegt in Rückenlage mit leicht erhöhtem Oberkörper.

Es erfolgt ein etwa $8 \mathrm{~cm}$ langer Hautschnitt median im oberen Sternumanteil, etwa von der 2. bis zur 4. Rippe. Anschließend wird das Sternum mittels Knochensäge eröffnet und mittels Thoraxsperrer offengehalten. Der Anschluss an die Herz-Lungen-Maschine erfolgt mittels Kanülierung der $A$. ascendens sowie bicavaler Kanülierung für den venösen Abfluss. Auch hier erfolgt die Insufflation von Kohlendioxid in das Operationsfeld sowie die Verabreichung der kardioplegen Lösung in die Aortenwurzel.

Nach Eröffnen des Perikards kann die Mitralklappe über verschiedene Wege erreicht werden. Im Folgenden werden die beiden an der Uniklinik Frankfurt im Wesentlichen angewandten und der Studie zugrunde liegenden Zugangswege beschrieben:

- transseptaler Zugang

- transcavaler Zugang

Transseptaler Zugang zur Mitralklappe nach partieller oberer Sternotomie: Hier wird zunächst der rechte Vorhof eröffnet. Von dort aus wird ein Längsschnitt durch das interatriale Septum bis hin zum linken Vorhofdach durchgeführt. Von dort besteht dann die beste Sicht auf die Mitralklappe. ${ }^{32}$ Dies bezeichnet man auch als superioren oder ausgedehnten vertikalen transseptalen Zugang. In der Literatur wird häufig auch der rein transseptale Zugang erwähnt, bei dem die Inzision auf das Septum begrenzt ist und sich nicht bis zum Vorhofdach hochzieht.

Dieser Zugang bringt die beste Exposition der Mitralklappe mit sich und zeigt sich vorteilhaft bei gleichzeitig indizierter Trikuspidalklappenrekonstruktion (TKR). 
Hier besteht jedoch das Risiko einer postoperativen Sinusknotendysfunktion aufgrund einer möglichen Verletzung der Sinusknotenarterie, die von der rechten Koronararterie über das Dach des rechten Vorhofes zieht. ${ }^{33,34}$ Die Frage nach diesbezüglich postoperativ auftretenden Rhythmusstörungen oder Indikation zur Schrittmacherimplantation soll in dieser Arbeit untersucht werden.

Transcavaler Zugang zur Mitralklappe nach partieller oberer Sternotomie Hier wird der rechte Vorhof intakt gelassen und anstelle dessen die Vena cava superior kurz vor Eintritt in den rechten Vorhof durchtrennt, um die Sicht auf die Mitralklappe zu verbessern. Die Eröffnung des linken Vorhofs erfolgt dann ebenfalls über das linke Vorhofdach und die interatriale Grube.

Erstmals beschrieben wurde der Zugang über die Vena cava superior von H. B. Barner (1985), und zwar als Kombination von superiorer Sternotomie und rechtslateralem Zugang bei nicht ausreichender Visualisierung der Mitralklappe nach rechtslateralem Zugang. ${ }^{35}$

\subsection{Vorhofflimmern}

\subsubsection{Epidemiologie}

Vorhofflimmern (VHF) ist die häufigste Form der Herzrhythmusstörung und zeigt insbesondere in der westlichen Welt eine erhöhte Prävalenz mit steigender Inzidenz. ${ }^{36}$

Die Inzidenz ist altersabhängig und steigt mit zunehmendem Lebensalter an. 37, 38 Patienten mit Komorbiditäten wie arteriellem Hypertonus, Herzinsuffizienz, koronarer Herzkrankheit, Herzklappenerkrankungen, Diabetes mellitus und chronischer Niereninsuffizienz sind häufiger betroffen. ${ }^{39}$ Auch zeigt sich ein Unterschied in den Geschlechtern: Männer sind häufiger betroffen als Frauen. ${ }^{40}$ Vorhofflimmern erhöht maßgeblich das Risiko für Schlaganfall und Herzinsuffizienz und geht mit einer verminderten Lebensqualität einher. ${ }^{37}$ 
Die Mortalität ist laut der 1998 durchgeführten Framingham-Studie 1,5- bis 1,9-fach so hoch wie bei Patienten mit Sinusrhythmus. ${ }^{41,42}$

\subsection{2 Ätiologie}

Vorhofflimmern kann sowohl primär bei Herzgesunden auftreten als auch sekundär durch kardiale und extrakardiale Ursachen wie Mitralklappenvitien, Koronare Herzkrankheit, Herzinfarkt, Herzinsuffizienz, Kardiomyopathien, arteriellen Hypertonus und Hyperthyreose. Auch eine genetische Prädisposition ist beschrieben. ${ }^{43}$

Eine eigene Entität ist das peri- und postoperative Vorhofflimmern. Insbesondere bei herzchirurgischen Operationen wie der Mitralklappen- und der Bypasschirurgie stellt Vorhofflimmern die häufigste postoperative Herzrhythmusstörung dar. Es tritt in bis zu 60 \% der Fälle auf und erhöht die Morbidität und Mortalität sowie die Hospitalisierungszeit. ${ }^{44}$

Das Risiko für postoperatives Vorhofflimmern ist insbesondere bei Mitralklappenoperationen erhöht. ${ }^{45}$ Des Weiteren spielen hohes Alter, ein erhöhter Body-mass-Index und das männliche Geschlecht eine risikoerhöhende Rolle. ${ }^{44}$

\subsubsection{Pathophysiologie}

Pathophysiologisch handelt es sich bei Vorhofflimmern um eine kreisende Erregung im linken Vorhof. Der Ursprung des paroxysmalen Vorhofflimmerns liegt in fokalen Erregungen im Bereich der Pulmonalvenen. ${ }^{46}$ Das persistierende Vorhofflimmern entspringt mehreren Reentry-Kreisläufen um Strukturen wie Klappenringe und Venenmündungen. Es kommt zu einer irregulären Dauererregung (Reentry-Tachykardie) des Vorhofs ohne hämodynamisch wirksame Vorhofkontraktion (absolute Arrhythmie). ${ }^{47}$ Durch den AV-Knoten mit seiner Funktion als Frequenzfilter kann sowohl eine Bradyarrhythmia absoluta als auch eine Tachyarrhythmia absoluta resultieren. 
Durch externe Stressoren wie arterielle Hypertonie, strukturelle Herzerkrankungen und Vorhofflimmern selbst wird ein strukturelles Remodeling induziert, das wiederum den Boden für permanentes Vorhofflimmern bietet. ${ }^{48}$

\subsubsection{Diagnostik}

Patienten mit Vorhofflimmern können sowohl asymptomatisch als auch symptomatisch sein. Meist äußert sich dies in Form von Palpitationen oder Herzrasen sowie mit hämodynamischer Instabilität bei akuter Dekompensation. Die Diagnose erfolgt mittels EKG. Hier zeigen sich unregelmäßige RR-Intervalle sowie fehlende P-Wellen. Um auch paroxysmales VHF detektieren zu können, ist ein Langzeit-EKG zielführend. Des Weiteren ist eine transthorakale Echokardiografie zur Beurteilung der Herzfunktion unverzichtbar. Laut ESC-Guidelines von 2016 erfolgt eine Einteilung anhand der Zeitdauer des Vorhofflimmerns:

- Erstmalig diagnostiziertes VHF

- Paroxysmales VHF (ehemals intermittierendes VHF): selbstlimitierend innerhalb 48 Std. bis 7 Tage

- Persistierendes VHF: > 7 Tage anhaltend

- Lang anhaltend persistierendes VHF: $\geq 1$ Jahr

- Permanentes VHF (ehemals chronisches VHF): akzeptiertes VHF, keine Rhythmisierungsversuche

\subsubsection{Therapie}

Die Therapie erfolgt individuell unter ganzheitlicher Betrachtung des Patienten. Bei Erstdiagnose des Vorhofflimmerns sind die hämodynamische Stabilität, die potenziell zugrunde liegende strukturelle Erkrankung, das Schlaganfallrisiko und eine notwendige Frequenz- oder Rhythmuskontrolle entscheidend für die Therapie. ${ }^{49}$ Zur Verfügung stehen medikamentöse, interventionelle und chirurgische Therapiemöglichkeiten. 


\subsubsection{Medikamentöse Therapie}

Die konventionelle Therapie des Vorhofflimmerns umfasst die Thromboembolieprophylaxe, die Frequenzkontrolle und die Rhythmuskontrolle. Zudem gilt es, die zugrunde liegende Erkrankung sowie Komorbiditäten suffizient zu behandeln, beispielsweise in Form einer optimalen Herzinsuffizienztherapie.

\subsection{Antikoagulation}

Aufgrund der fehlenden effektiven Vorhofkontraktion mit Blutstase besteht ein erhöhtes Risiko von Thrombenbildung im Vorhof. ${ }^{50}$ Die daraus resultierende hohe Zahl durch Vorhofflimmern verursachter cerebraler Insulte kann durch eine orale Antikoagulation nachweislich reduziert werden. ${ }^{51,49}$ Gleichzeitig steigt jedoch das Risiko für lebensgefährliche Blutungsereignisse.

Das individuelle Schlaganfallrisiko und die Indikation zur Antikoagulation wird anhand des CHA2DS2-Vasc-Score beurteilt. ${ }^{52}$

\begin{tabular}{|l|l|l|}
\hline C & Congestive heart failure & 1 \\
H & Hypertension $(>140 / 90 \mathrm{mmHg})$ & 1 \\
A2 & Age $\geq 75$ & 2 \\
D & Diabetes mellitus & 1 \\
S2 & Prior TIA or stroke & 2 \\
V & Vascular disease (myocardial infarction, aortic & 1 \\
& plaque etc.) & \\
A & Age 65-74 & 1 \\
Sc & Sex category (Female=1 pt.) & 1 \\
\hline
\end{tabular}

Abbildung 1: CHA2DS2-Vasc-Score

Ab einem Ergebnis von 2 Punkten bei Männern und 3 Punkten bei Frauen wird aufgrund des erhöhten Risikos die Antikoagulation mittels Vitamin-KAntagonisten oder NOAKs zur Thromboembolieprophylaxe empfohlen. Bei Patienten mit 1 (Männer) oder 2 (Frauen) Punkten ist eine ausführliche RisikoNutzen-Abwägung durchzuführen. 
Bei Kontraindikation zur Antikoagulation, bspw. eine vorangegangene Hirnblutung, kann ein Vorhofohrverschluss vor Thrombenbildung schützen. Hierbei wird das Herzohr, das der häufigste Entstehungsort der durch Vorhofflimmern induzierten Thromben ist, entweder chirurgisch oder minimalinvasiv mittels Applikationskatheter durch einen sogenannten Watchman-Okkluder verschlossen. ${ }^{53-55}$

\subsection{Frequenzkontrolle}

Die Frequenzkontrolle dient der Symptomlinderung bei tachykardem Vorhofflimmern sowie der Stabilisierung der Herzfunktion. Ziel ist das Erreichen eines normofrequenten Herzrhythmus. Zum Einsatz kommen Betablocker sowie Calziumantagonisten vom Nicht-Dihydropyridin-Typ und Digitalispräparate. Bei bradykard übergeleitetem Vorhofflimmern kann die Implantation eines Herzschrittmachers eine adäquate Pumpfunktion des Herzens sichern. ${ }^{56}$

\subsection{Rhythmuskontrolle}

Unter Rhythmuskontrolle versteht man die Herbeiführung der Konversion in einen Sinusrhythmus. Im Hinblick auf das Outcome zeigt sich keine Überlegenheit gegenüber der Frequenzkontrolle. ${ }^{57,58}$ Die Indikation besteht in der Symptomverbesserung des Patienten.

In der Akutsituation bei hämodynamisch relevanter Tachyarrhythmia absoluta kann dies durch eine elektrische oder medikamentöse Kardioversion erfolgen. Ebenso kann diese elektiv bei symptomatischen Patienten durchgeführt werden, sofern zuvor ein Thrombenausschluss mittels transösophagealer Echokardiografie oder eine dreiwöchige Antikoagulation erfolgt ist. ${ }^{59}$ Bei der medikamentösen Kardioversion kommen Antiarrhythmika der Klasse I und III zur Anwendung. Der Erfolg der Kardioversion hängt von der Dauer des Vorhofflimmerns ab. Je länger das Vorhofflimmern besteht, desto geringer die Konversionswahrscheinlichkeit und desto höher die Rezidivraten. ${ }^{60}$ 
Die Rezidivwahrscheinlichkeit nach elektrischer Kardioversion beträgt $75 \%$ innerhalb des ersten Jahres. Unter additiver medikamentöser antiarrhythmischer Therapie kann der Erfolg der Elektrokardioversion verbessert werden. ${ }^{61,62}$

\subsubsection{Ablationsverfahren}

Einen weiteren potenziell kurativen Therapieansatz liefern die in den vergangenen Jahren entwickelten Ablationsverfahren. Diese kommen bei Versagen der medikamentösen Therapie oder bei ausgewählten stark symptomatischen Patienten mit paroxysmalem VHF zum Einsatz. Zur Verfügung stehen minimalinvasive Ablationsverfahren mittels eines Katheters über die Leiste sowie die chirurgische Ablation.

\subsection{Katheterablation}

Bei der Katheterablation werden mittels Kälte- oder Hitzeenergie die in einer elekrophysiologischen Untersuchung identifizierten Foki verödet und ggf. eine Pulmonalvenenisolation durchgeführt. ${ }^{63}$ Die Erfolgsrate ist höher, je früher der Eingriff durchgeführt wird. Bei paroxysmalem Vorhofflimmern beträgt die Erfolgsrate bei $70-80 \%$, bei persistierendem Vorhofflimmern $50-70 \%$, bei permanentem VHF $50 \%{ }^{64}$

Als Ultima Ratio kann eine Ablation des AV-Knotens zur Regulation der Kammerfrequenz führen. Dies bringt jedoch die Notwendigkeit eines Herzschrittmachers mit sich und führt lediglich zu einer Verbesserung der Symptomatik und der linksventrikulären Funktion mit erhöhter körperlicher Belastbarkeit, aber nicht zu einer Beseitigung des Vorhofflimmerns. ${ }^{65}$

\subsection{Chirurgische Ablation}

Die chirurgischen Ablationsverfahren werden in der Regel in Kombination mit anderen herzchirurgischen Operationen durchgeführt, im Kontext der vorliegenden Arbeit im Rahmen von Mitralklappenoperationen. 
Die ursprüngliche chirurgische Therapie des Vorhofflimmerns in Form der cutand-sew-Methode der Maze-Prozedur wurde erstmals 1991 von James Cox beschrieben.

Bei dieser Methode wurden in beiden Vorhöfen nach einem bestimmten Muster multiple Inzisionen getätigt und anschließend wieder vernäht.

Das so geschaffene Narbengewebe stellt einen Leitungsblock dar und verhindert die kreisenden Erregungen, die zum Vorhofflimmern führen. Es entsteht ein Labyrinth, begrenzt durch nicht leitfähiges Gewebe (Maze = Labyrinth). Durch dieses Labyrinth wird nur noch die geordnete physiologische Erregungsausbreitung vom Sinusknoten zum AV-Knoten weitergeleitet. Das Verfahren wurde über die Jahre mehrfach modifiziert und mündete schließlich in der MAZE-III-Operation. Diese verbesserte die Ergebnisse hinsichtlich des postoperativen Sinusrhythmus, der Sinusknotenfunktion, geringerer Rezidivraten und geringerer Zahl an postoperativer Schrittmacherpflichtigkeit. ${ }^{47}$

Die Methode wurde zur Methode der Wahl für therapierefraktäre symptomatische Patienten mit Vorhofflimmern. ${ }^{66}$ Mit Fortschreiten der Zeit fanden weitere Modifikationen statt. Ziel war es, Alternativen für die zwar sehr effektive, jedoch komplexe chirurgische Schnittführung mit hoher Schrittmachernotwendigkeit zu finden.

Mit Entwicklung sogenannter Ablationstools wurden die chirurgischen Inzisionen durch einen Ablationsstift oder eine Ablationszange ersetzt, die mittels Kryo-oder Radiofrequenz (RF bzw. Hochfrequenz= HF) ohne anschließendes Vernähen den Vernarbungseffekt erzielen. Des Weiteren wurde die Art der Pulmonalvenenisolation modifiziert. Statt alle vier Pulmonalvenen mithilfe eines Schnittes zu umschließen und damit auch den linken Vorhof ungewollt elektrisch zu isolieren, erfolgte die Ablation der Pulmonalvenen nun paarweise mit einer einzelnen Verbindungslinie. Das Resultat war eine bessere Vorhofaktivität unter Vermeidung einer Akinesie durch die elektrische Isolation. ${ }^{67,68}$ 
So erfolgte die Weiterentwicklung immer mehr in Richtung einer minimalinvasiveren Methode (Maze-IV), die auch isoliert ohne weitere herzchirurgische Intervention endoskopisch durchgeführt werden kann. ${ }^{69}$

Die Läsionen mittels Kälte- oder Hitzeenergie werden von endo- oder epikardial durchgeführt. Im Falle der hier untersuchten Mitralklappenoperationen wurde fast ausschließlich von endokardial abladiert, da der Vorhof für die Mitralklappenoperation ohnehin eröffnet ist. Epikardial wird abladiert, wenn für die Hauptoperation der Vorhof nicht eröffnet werden muss (Aortenklappen-OP, Bypass-OP).

Die ursprüngliche cut-and-sew-Methode lieferte zwar die bessere Rhythmusstabilität im Vergleich zur Ablation (Durchtrennen ist wirksamer als Veröden), war jedoch auch wesentlich komplexer in der Durchführung.

Die Erfolgsrate der chirurgischen Ablation hängt maßgeblich von der Dauer des persistierenden VHF und der Größe des linken Vorhofs ab. ${ }^{70}$ Bei guten Bedingungen (kleines linkes Atrium, kurze Dauer des persistierenden VHF) kommt es bei etwa $90 \%$ der Patienten zur Konversion in den Sinusrhythmus. ${ }^{71}$ Im Vergleich zur Katheterablation ist jedoch ein deutlich höheres Risiko aufgrund der größeren Invasivität des Eingriffs mit der Notwendigkeit einer Vollnarkose und Herz-Lungen-Maschine zu beachten. Das Risiko für eine Schrittmacherpflichtigkeit besteht bei beiden Verfahren, schon allein durch das Vorhofflimmern selbst, das zu einer Schädigung des Sinusknotens führen kann. Für Patienten mit persistierendem oder lang anhaltendem Vorhofflimmern zeigte die bei der Mitralklappenoperation durchgeführte Ablation eine deutliche Reduktion an Vorhofflimmern innerhalb des ersten postoperativen Jahres, jedoch erhöhte sich die Wahrscheinlichkeit einer Schrittmacherpflichtigkeit. ${ }^{72,73}$ 
Zusammenfassend kann man festhalten, dass in den vergangenen Jahren sowohl die operativen Zugangswege zur Mitralklappe als auch die Ablationsmöglichkeiten bei Vorhofflimmern ständig modifiziert und optimiert wurden.

Inwiefern sich die an der Universität Frankfurt genutzten minimalinvasiven Zugangswege und die additive chirurgische Ablation im Hinblick auf den postoperativen Herzrhythmus auswirken, soll in dieser Studie untersucht werden. 


\section{Fragestellung}

Die vorliegende Arbeit befasst sich mit dem Einfluss minimalinvasiver Zugangswege zur Mitralklappe und der damit verbundenen unterschiedlichen Vorhofinzisionen auf den postoperativen Herzrhythmus. Die hier untersuchten minimalinvasiven Zugangswege waren die anterolaterale Minithorakotomie mittels Chitwood-Klemme und die partielle obere Sternotomie mit transcavalem und transseptalem Zugang.

Von Interesse ist dabei, ob sich die geschilderten drei genutzten Zugangswege im Hinblick auf folgende Aspekte unterscheiden:

- Inzidenz von Vorhofflimmern bei präoperativem Sinusrhythmus

- Ablationserfolg (Konversion in den Sinusrhythmus) bei präoperativ bestehendem Vorhofflimmern

- Inzidenz von Schrittmacherimplantationen

- Überleben 


\section{Material und Methoden}

\subsection{Patientenauswahl}

In die Studie eingeschlossen wurden alle Patienten, die sich zwischen 1998 und 2015 in der Klinik für Thorax-, Herz- und thorakale Gefäßchirurgie der Universitätsklinik Frankfurt am Main unter der Leitung von Prof. Dr. med. Moritz einer Mitralklappenoperation über die genannten minimalinvasiven Zugangswege unterzogen. Dabei wurden sowohl Mitralklappenrekonstruktionen als auch der Mitralklappenersatz durchgeführt, außerdem verschiedene Kombinationseingriffe.

Ein Ausschluss erfolgte nur auf ausdrücklichen Wunsch des Patienten.

\subsection{Datenerhebung}

Es handelt sich bei der vorliegenden Arbeit um eine prospektive Studie. Die Datenerhebung erfolgte anhand interner und externer Befunde sowie aus Krankenakten des stationären und ambulanten Aufenthaltes in der Universitätsklinik Frankfurt am Main.

Die präoperativen Daten entstammten Befunden aus der THG-Ambulanz (Aufnahmeecho) sowie zuweisender niedergelassener Kardiologen. Postoperative Daten wurden der Krankenakte des stationären Aufenthaltes sowie Befunden vorhandener Anschlussheilbehandlungen entnommen.

Die Nachuntersuchung erfolgte in Intervallen mit Hilfe eines standardisierten Fragebogens (im Anhang) sowie anhand der Nachuntersuchungsbefunde der THG-Ambulanz. Ein erheblicher Teil der Patienten wurde hier echokardiografisch untersucht. Vervollständigt wurden diese Angaben durch telefonisch angeforderte externe Befunde niedergelassener Kardiologen oder Hausärzte.

Die Studie wurde von der Ethikkommission des Frankfurter Universitätszentrums mit den Voten 387/12 und 97/13 genehmigt. 


\section{Datenerfassung und Auswertung}

\subsection{Datenerfassung und Datenspeicherung}

Die Datenerfassung erfolgte aus den Patientenakten, zunächst in Papierform und später in Form digitalisierter Akten mit dem Programm Orbis. Die Aufnahme in eine wissenschaftliche Datenbank erfolgte mit dem Programm FileMaker. Aus dieser Datenbank konnte ein pseudonymisierter Export in Excel durchgeführt werden, um die statistische Auswertung zu ermöglichen.

\subsection{Statistische Auswertung}

Die statistische Auswertung wurde mit dem Programm SPSS für Mac durchgeführt. Tabellen und Diagramme wurden mit dem Programm Microsoft Excel erstellt.

Der Vergleich zwischen den drei nach den genannten Zugangswegen eingeteilten Patientengruppen erfolgte zunächst mittels Globaltest. Auf Normalverteilung wurde mit dem Kolmogorov-Smirnov-Test geprüft. Als Globaltest bei kategorialen Variablen wurde der Chi-Quadrat-Test verwendet, bei ordinalen Variablen der Kruskal-Wallis-Test. Bei signifikantem Globaltest wurden Paarvergleiche zwischen je zwei Gruppen durchgeführt, um den signifikanten Unterschied zu lokalisieren. Dafür wurde bei kategorialen Variablen der Chi-Quadrat-Test verwendet, bei ordinalen Variablen der MannWhitney-Test. Bei Werten $<5$ einer Variablen wurde der Fischer-Exakt-Test angewandt. Als statistisch signifikanter Wert wurde ein $p$-Wert $<0,05$ definiert. Die Korrektur der $p$-Werte nach Mehrfachtestung erfolgte nach BonferroniHolm.

Die Darstellung des Überlebens sowie der Freiheit von Ereignissen erfolgte mittels Kaplan-Meier-Schätzer. Die Signifikanz wurde dabei mit dem Log-RankTest überprüft. 
Um verschiedene Einflussfaktoren auf eine Zielvariable zu untersuchen, wurden multivariate Analysen durchgeführt.

Durchgeführt wurden auch eine logistische Regression für den Einfluss von unabhängigen Variablen auf den Ablationserfolg sowie eine Cox-Regression für den Einfluss von unabhängigen Variablen auf die Zahl der Schrittmacherimplantationen. 


\section{Ergebnisse}

Im Folgenden werden die Ergebnisse der statistischen Auswertung geschildert. Zunächst erfolgt die Beschreibung des präoperativen Status des Patientenkollektivs. Anschließend werden intraoperative und postoperative Daten sowie Langzeitergebnisse des Follow-ups beschrieben. Im Anschluss folgt eine zusätzliche Auswertung mit einem Patientenkollektiv nach Propensity Score Matching, um vorhandene Unterschiede in den Untersuchungsgruppen zu egalisieren.

\subsection{Studienpopulation}

Das Patientenkollektiv umfasst alle Patienten, die sich im Zeitraum 1. Januar 1998 bis 31. Dezember 2015, an der Uniklinik Frankfurt am Main in der Klinik für Herz-, Thorax- und thorakale Gefäßchirurgie einer Mitralklappenoperation (Mitralklappenrekonstruktion oder Mitralklappenersatz) über einen der im Folgenden genannten minimalinvasiven Zugangswege unterzogen haben. Daraus resultiert eine Patientenanzahl von 1206 Patienten für die vorliegende Studie. Bei 300 Patienten wurde die anterolaterale Minithorakotomie mit Aortenklemmung mittels Chitwood-Klemme, bei den verbleibenden 906 Patienten die partielle obere Sternotomie angewandt. In den beiden Gruppen mit partieller oberer Sternotomie wurde bei 687 Patienten ein transseptaler und bei 219 Patienten ein transcavaler Zugang zur Mitralklappe gewählt. Im Folgenden werden die verschiedenen Gruppen der Übersichtlichkeit wegen als

- Gruppe A für den Chitwood-Zugang

- Gruppe B für die POS transseptal

- Gruppe C für die POS transcaval

bezeichnet und im Vergleich zueinander aufgeführt. 


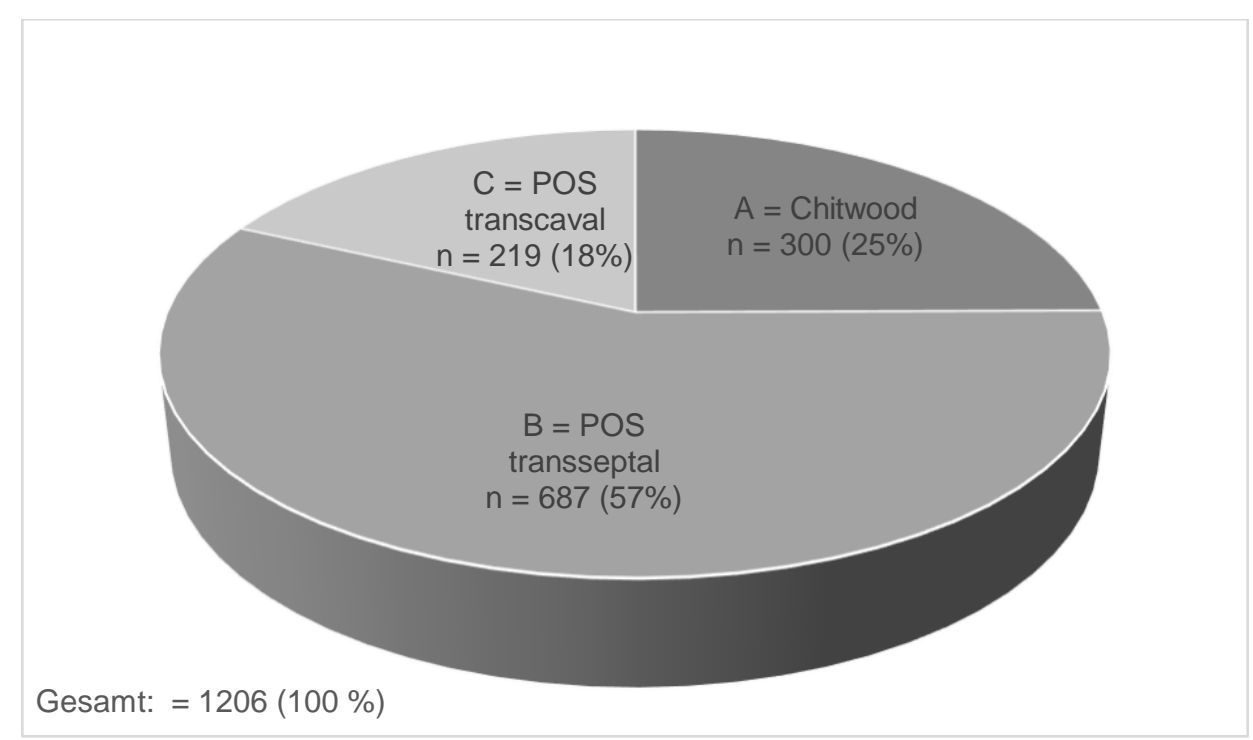

Abbildung 2: Häufigkeit der Zugangswege im Patientenkollektiv

\subsubsection{Alter und Geschlecht}

Die Studienpopulation war zu $58 \%$ männlichen und zu $42 \%$ weiblichen Geschlechts. Der Gruppe A gehörten mehr Frauen an, in Gruppe B und C stellten die Männer den größeren Anteil dar.

Das Durchschnittsalter des gesamten Patientenkollektivs zum Zeitpunkt der Operation betrug $55 \pm 14$ Jahre, wobei die Patienten in Gruppe A am ältesten und die in Gruppe B am jüngsten waren.

Hinsichtlich des Alters und des Geschlechts gab es signifikante Unterschiede zwischen den Gruppen.

\begin{tabular}{|l|l|l|l|l|l|l|}
\hline & $\begin{array}{l}\text { Gruppe A } \\
\mathbf{n}=300\end{array}$ & $\begin{array}{l}\text { Gruppe B } \\
\mathbf{n = 6 8 7}\end{array}$ & $\begin{array}{l}\text { Gruppe C } \\
\mathbf{n = 2 1 9}\end{array}$ & $\begin{array}{l}\text { Gesamt } \\
\mathbf{n = 1 2 0 6}\end{array}$ & $\begin{array}{l}\text { p-Wert } \\
\text { (Globaltest) }\end{array}$ & $\begin{array}{l}\text { p-Wert } \\
\text { (Paarvergleiche) }\end{array}$ \\
\hline Geschlecht & & & & & $<0,0001$ & $\begin{array}{l}\text { A-B: }<\mathbf{0 , 0 0 0 1} \\
\text { A-C: }<0,0001 \\
\text { B-C: }<0,0001\end{array}$ \\
\hline männlich & $139(46,3 \%)$ & $409(59,5 \%)$ & $152(69,4 \%)$ & $700(58,0 \%)$ & & \\
\hline weiblich & $161(53,7 \%)$ & $278(40,5 \%)$ & $67(30,6 \%)$ & $506(42,0 \%)$ & & \\
\hline
\end{tabular}




\begin{tabular}{|c|c|c|c|c|c|c|}
\hline Alter (Jahre) & & & & & $<0,0001$ & $\begin{array}{l}\text { A-B: }<0,0001 \\
\text { A-C: }<0,0001 \\
\text { B-C: } 0,008\end{array}$ \\
\hline Mittelwert \pm SD & $64 \pm 13$ & $57 \pm 13$ & $61 \pm 14$ & $55 \pm 14$ & & \\
\hline Minimum & 16 & 18 & 14 & 14 & & \\
\hline Maximum & 88 & 82 & 88 & 81 & & \\
\hline
\end{tabular}

Tabelle 1: Geschlecht und Alter der Studienpopulation

\subsection{Präoperative Daten}

In diesem Abschnitt werden die extern und intern erhobenen präoperativen Befunde erläutert. Entnommen wurden diese aus der elektronischen Akte.

\subsubsection{Kardialer präoperativer Status}

Insgesamt waren 12,2 \% der Patienten an einer koronaren Herzkrankheit vorerkrankt. Die meisten von innen (6,8 \%) hatten eine 1-Gefäß-KHK, 2,8 \% eine 2-Gefäß-KHK und nur 1,3 \% eine 3-Gefäß-KHK. In diesem Punkt gab es keinen signifikanten Unterschied zwischen den Gruppen.

Einen signifikanten Unterschied konnte man in der Prävalenz von pulmonaler Hypertonie zwischen den Gruppen feststellen. In Gruppe B hatten 64,6\% der Patienten einen pulmonalen Hypertonus, in Gruppe A und $\mathrm{C}$ waren es $35 \%$ und $30 \%$.

Der Durchmesser des linken Vorhofs (LA-Größe) betrug insgesamt im Mittelwert 49,7 mm \pm 9,1 Standardabweichung; darin unterschieden sich die Gruppen nicht signifikant.

\begin{tabular}{|l|l|l|l|l|l|l|}
\hline & $\begin{array}{l}\text { Gruppe A } \\
\mathbf{n}=\mathbf{3 0 0}\end{array}$ & $\begin{array}{l}\text { Gruppe B } \\
\mathbf{n = 6 8 7}\end{array}$ & $\begin{array}{l}\text { Gruppe C } \\
\mathbf{n = 2 1 9}\end{array}$ & $\begin{array}{l}\text { Gesamt } \\
\mathbf{n = 1 2 0 6}\end{array}$ & $\begin{array}{l}\mathbf{p} \text {-Wert } \\
\text { (Globaltest) }\end{array}$ & $\begin{array}{l}\text {-Wert } \\
\text { (Paarvergleiche) }\end{array}$ \\
\hline KHK & $\begin{array}{l}35(12,2 \% \\
\text { von } \mathrm{n}=288)\end{array}$ & $\begin{array}{l}81(11,8 \% \\
\text { von } \mathrm{n}=684)\end{array}$ & $\begin{array}{l}14(6,4 \% \\
\text { von } \mathrm{n}=219)\end{array}$ & $\begin{array}{l}130(12,2 \% \\
\text { von } \mathrm{n}=1191)\end{array}$ & $\mathbf{0 , 2 5 8}$ & \\
\hline $1-\mathrm{G}$ & $24(8,3 \%)$ & $48(7,0 \%)$ & $9(4,1 \%)$ & $81(6,8 \%)$ & & \\
\hline $2-G$ & $6(2,1 \%)$ & $23(3,4 \%)$ & $5(1,8 \%)$ & $33(2,8 \%)$ & & \\
\hline
\end{tabular}




\begin{tabular}{|l|l|l|l|l|l|l|}
\hline 3-G & $5(1,7 \%)$ & $10(1,5 \%)$ & $1(0,5 \%)$ & $16(1,3 \%)$ & & \\
\hline $\begin{array}{l}\text { Pulmonaler } \\
\text { Hypertonus }\end{array}$ & $\begin{array}{l}101(35,2 \% \\
\text { von } \mathrm{n}=286)\end{array}$ & $\begin{array}{l}430(64,6 \% \\
\text { von } \mathrm{n}=666)\end{array}$ & $\begin{array}{l}66(30,7 \% \\
\text { von } \mathrm{n}=215)\end{array}$ & $\begin{array}{l}597(51,1 \% \\
\text { von } \mathrm{n}=1167)\end{array}$ & $<\mathbf{0 , 0 0 0 1}$ & $\begin{array}{l}\text { A-B: }<\mathbf{0 , 0 0 1} \\
\text { A-C: } \mathbf{0 , 4 0 1} \\
\text { B-C: }<\mathbf{0 , 0 0 1}\end{array}$ \\
\hline gering & $60(21,0 \%)$ & $153(23,0 \%)$ & $33(15,3 \%)$ & $246(21,1 \%)$ & & \\
\hline mittel & $25(8,7 \%)$ & $143(21,5 \%)$ & $21(9,8 \%)$ & $189(16,2 \%)$ & & \\
\hline hochgradig & $16(5,6 \%)$ & $134(20,1 \%)$ & $12(5,6 \%)$ & $162(13,9 \%)$ & & \\
\hline $\begin{array}{l}\text { LA-Größe } \\
\text { (mm) }\end{array}$ & $\begin{array}{l}\text { Mittelwert } \pm \\
\text { SD }\end{array}$ & $\begin{array}{l}50,3 \pm 10,1 \\
(\mathrm{n}=177)\end{array}$ & $\begin{array}{l}49,5 \pm 8,8 \\
(\mathrm{n}=448)\end{array}$ & $\begin{array}{l}49,8 \pm 9,1 \\
(\mathrm{n}=130)\end{array}$ & $\begin{array}{l}49,7 \pm 9,1 \\
(\mathrm{n}=755)\end{array}$ & \\
\hline
\end{tabular}

Tabelle 2: Kardialer präoperativer Status

In den folgenden beiden Tabellen wird der präoperative Herzrhythmus dargestellt, insbesondere im Hinblick auf präoperativ bestehendes Vorhofflimmern sowie die Prävalenz von Herzschrittmachern im Patientenkollektiv. In beiden Aspekten konnten signifikante Unterschiede zwischen den drei Gruppen ermittelt werden. Der Anteil an Vorhofflimmern war in der Gruppe B am höchsten und in Gruppe C am geringsten. Das Vorhofflimmern bestand in den meisten Fällen chronisch mit $25 \%$, bei 14,8 \% handelte es sich um intermittierendes Vorhofflimmern.

\begin{tabular}{|l|l|l|l|l|l|l|}
\hline & $\begin{array}{l}\text { Gruppe A } \\
\mathbf{n = 3 0 0}\end{array}$ & $\begin{array}{l}\text { Gruppe B } \\
\mathbf{n = 6 8 7}\end{array}$ & $\begin{array}{l}\text { Gruppe C } \\
\mathbf{n = 2 1 9}\end{array}$ & $\begin{array}{l}\text { Gesamt } \\
\mathbf{n = 1 2 0 6}\end{array}$ & $\begin{array}{l}\text { p-Wert } \\
\text { (Globaltest) }\end{array}$ & $\begin{array}{l}\text { p-Wert } \\
\text { (Paarvergleiche) }\end{array}$ \\
\hline $\begin{array}{l}\text { Präoperativer } \\
\text { Herzrhythmus }\end{array}$ & & & & $\mathbf{0 , 0 0 1}$ & $\begin{array}{l}\text { A-B: } \mathbf{0 , 0 1 2 6} \\
\text { A-C: } \mathbf{0 , 0 1 2 6} \\
\text { B-C: }<\mathbf{0 , 0 0 1}\end{array}$ \\
\hline SR & $186(62,0 \%)$ & $387(56,3 \%)$ & $153(69,9 \%)$ & $726(60,2 \%)$ & & \\
\hline VHF & $114(38,0 \%)$ & $300(43,7 \%)$ & $66(30,1 \%)$ & $480(39,8 \%)$ & & \\
\hline Intermittierend & $43(14,3 \%)$ & $111(16,2 \%)$ & $25(11,4 \%)$ & $179(14,8 \%)$ & & \\
\hline Chronisch & $71(23,7 \%)$ & $189(27,5 \%)$ & $41(18,7 \%)$ & $301(25,0 \%)$ & & \\
\hline
\end{tabular}

Tabelle 3: Präoperativer Herzrhythmus 
Von allen 1206 Patienten hatten 5,6 \% bereits präoperativ einen Herzschrittmacher, wovon in Gruppe B der größte Anteil vorkam.

\begin{tabular}{|l|l|l|l|l|l|l|}
\hline & $\begin{array}{l}\text { Gruppe A } \\
\mathbf{n = 3 0 0}\end{array}$ & $\begin{array}{l}\text { Gruppe B } \\
\mathbf{n = 6 8 7}\end{array}$ & $\begin{array}{l}\text { Gruppe C } \\
\mathbf{n = 2 1 9}\end{array}$ & $\begin{array}{l}\text { Gesamt } \\
\mathbf{n = 1 2 0 6}\end{array}$ & $\begin{array}{l}\mathbf{p} \text {-Wert } \\
\text { (Globaltest) }\end{array}$ & $\begin{array}{l}\mathbf{p} \text {-Wert } \\
\text { (Paarvergleiche) }\end{array}$ \\
\hline $\begin{array}{l}\text { Schrittmacher } \\
\text { präoperativ }\end{array}$ & $10(3,3 \%)$ & $53(7,7 \%)$ & $5(2,3 \%)$ & $68(5,6 \%)$ & $\mathbf{0 , 0 0 1}$ & $\begin{array}{l}\text { A-B: } \mathbf{0 , 0 2 0} \\
\text { A-C: } \mathbf{0 , 4 8 1} \\
\text { B-C: } \mathbf{0 , 0 1 2}\end{array}$ \\
\hline
\end{tabular}

Tabelle 4: Patienten mit präoperativem Herzschrittmacher

\subsubsection{Voroperationen}

Insgesamt waren 77 der 1206 Patienten am Herzen voroperiert. Dabei kamen sowohl offene als auch minimalinvasive kathetergestützte Interventionen zum Einsatz. Am häufigsten hatten Patienten eine perkutane transluminale Koronarangioplastie (PTCA) in der Anamnese, gefolgt von einer Valvuloplastie. In der Gruppe A gab es den größten Anteil voroperierter Patienten mit 9,3\%, in Gruppe C mit 2,7 \% den Kleinsten. Zwischen diesen beiden Gruppen zeigte sich ein signifikanter Unterschied.

An der Mitralklappe waren 7 Patienten durch ein offen chirurgisches Verfahren voroperiert. Alle 7 Patienten gehörten zu Gruppe A, wovon 3 Patienten eine Mitralklappenrekonstruktion und 4 Patienten einen Mitralklappenersatz bekommen hatten.

\begin{tabular}{|l|l|l|l|l|l|l|}
\hline & $\begin{array}{l}\text { Gruppe A } \\
\mathbf{n = 3 0 0}\end{array}$ & $\begin{array}{l}\text { Gruppe B } \\
\mathbf{n = 6 8 7}\end{array}$ & $\begin{array}{l}\text { Gruppe C } \\
\mathbf{n = 2 1 9}\end{array}$ & $\begin{array}{l}\text { Gesamt } \\
\mathbf{n = 1 2 0 6}\end{array}$ & $\begin{array}{l}\text { p-Wert } \\
\text { (Globaltest) }\end{array}$ & $\begin{array}{l}\text { p-Wert } \\
\text { (Paarvergleiche) }\end{array}$ \\
\hline $\begin{array}{l}\text { Kardiale } \\
\text { Voroperationen }\end{array}$ & $28(9,3 \%)$ & $39(5,7 \%)$ & $6(2,7 \%)$ & $77(6,4 \%)$ & $\mathbf{0 , 0 1 0}$ & $\begin{array}{l}\text { A-B: } \mathbf{0 , 0 9 0} \\
\text { A-C: } \mathbf{0 , 0 0 9} \\
\text { B-C: } \mathbf{0 , 0 9 0}\end{array}$ \\
\hline $\begin{array}{l}\text { Geschlossen / } \\
\text { Katheterintervention }\end{array}$ & & & & & & \\
\hline Mitra-Clip & $0(0,0 \%)$ & $4(0,6 \%)$ & $0(0,0 \%)$ & $4(0,3 \%)$ & & \\
\hline (Ballon-) Valvuloplastie & $1(0,3 \%)$ & $9(1,3 \%)$ & $0(0,0 \%)$ & $10(0,8 \%)$ & & \\
\hline Mitralklappensprengung & $3(1,0 \%)$ & $3(0,4 \%)$ & $0(0,0 \%)$ & $6(0,5 \%)$ & & \\
\hline
\end{tabular}




\begin{tabular}{|l|l|l|l|l|l|l|}
\hline PTCA mit Stent & $6(2,0 \%)$ & $17(2,5 \%)$ & $2(0,9 \%)$ & $25(2,1 \%)$ & & \\
\hline Z. n. Ablation & $0(0,0 \%)$ & $1(0,1 \%)$ & $0(0,0 \%)$ & $1(0,08 \%)$ & & \\
\hline PDA-Verschluss & $0(0,0 \%)$ & $1(0,1 \%)$ & $1(0,5 \%)$ & $2(0,2 \%)$ & & \\
\hline PFO-Verschluss & $0(0,0 \%)$ & $1(0,1 \%)$ & $2(0,9 \%)$ & $3(0,2 \%)$ & & \\
\hline Herzohrverschluss & $0(0,0 \%)$ & $2(0,3 \%)$ & $0(0,0 \%)$ & $2(0,1 \%)$ & & \\
\hline Offen chirurgisch & $3(1,0 \%)$ & $0(0,0 \%)$ & $0(0,0 \%)$ & $3(0,2 \%)$ & & \\
\hline MKR & $4(1,3 \%)$ & $0(0,0 \%)$ & $0(0,0 \%)$ & $4(0,3 \%)$ & & \\
\hline MKE & $3(1,0 \%)$ & $0(0,0 \%)$ & $0(0,0 \%)$ & $3(0,2 \%)$ & & \\
\hline AKE & $6(2,0 \%)$ & $0(0,0 \%)$ & $1(0,5 \%)$ & $7(0,6 \%)$ & & \\
\hline ACB & $0(0,0 \%)$ & $1(0,1 \%)$ & $0(0,0 \%)$ & $1(0,08 \%)$ & & \\
\hline $\begin{array}{l}\text { Offene } \\
\text { Mitralklappensprengung }\end{array}$ & $1(0,3 \%)$ & $0(0,0 \%)$ & $0(0,0 \%)$ & $1(0,08 \%)$ & & \\
\hline Offene Valvulotomie & $1(0,3 \%)$ & $0(0,0 \%)$ & $0(0,0 \%)$ & $1(0,08 \%)$ & & \\
\hline $\begin{array}{l}\text { Z. n. Polytrauma mit } \\
\text { Lungenquetschung }\end{array}$ & & & & & \\
\hline
\end{tabular}

Tabelle 5: Kardiale Voroperationen

\subsection{Perioperative Daten}

In diesem Abschnitt werden alle Daten zur Operation und zu frühpostoperativen Ereignissen dargestellt.

\subsubsection{Art des Eingriffs und Kombinationseingriffe}

Bei den 1206 Mitralklappenoperationen handelte es sich in 87,2 \% der Fälle um eine Mitralklappenrekonstruktion und in 12,8 \% um einen Mitralklappenersatz. Ein Mitralklappenersatz wurde im Verhältnis am häufigsten in Gruppe A durchgeführt. Der Anteil an Mitralklappenrekonstruktionen war in Gruppe $C$ am größten. Dieser Unterschied zwischen den Gruppen war signifikant. In einigen Fällen wurden zusätzlich zur Mitralklappe weitere operative Eingriffe durchgeführt. Die beiden größten Gruppen bildeten dabei zusätzlich durchgeführte Trikuspidalklappenrekonstruktionen bei 25,4 \% und die chirurgische Ablation bei $19,4 \%$ der Patienten. 
Eine genaue Aufschlüsselung der Eingriffsart kann Tabelle 6 entnommen werden. Die meisten Kombinationseingriffe wurden in Gruppe B durchgeführt. In den Paarvergleichen zeigte sich ein signifikanter Unterschied von Gruppe B zu den Gruppen A und C.

\begin{tabular}{|c|c|c|c|c|c|c|}
\hline & $\begin{array}{l}\text { Gruppe A } \\
n=300\end{array}$ & $\begin{array}{l}\text { Gruppe B } \\
n=687\end{array}$ & $\begin{array}{l}\text { Gruppe C } \\
n=219\end{array}$ & $\begin{array}{l}\text { Gesamt } \\
n=1206\end{array}$ & $\begin{array}{l}\text { p-Wert } \\
\text { (Globaltest) }\end{array}$ & $\begin{array}{l}\text { p-Wert } \\
\text { (Paarvergleiche) }\end{array}$ \\
\hline $\begin{array}{l}\text { Mitralklappen- } \\
\text { rekonstruktion } \\
\text { und - ersatz }\end{array}$ & & & & & 0,010 & $\begin{array}{l}\text { A-B: } 0,159 \\
\text { A-C: } 0,002 \\
\text { B-C: } 0,022\end{array}$ \\
\hline MKR & $251(83,7 \%)$ & $598(87,0 \%)$ & $203(92,7 \%)$ & $1052(87,2 \%)$ & & \\
\hline MKE & $49(16,3 \%)$ & $89(13,0 \%)$ & $16(7,3 \%)$ & $154(12,8 \%)$ & & \\
\hline $\begin{array}{l}\text { Zusätzlicher } \\
\text { Klappeneingriff }\end{array}$ & $16(5,3 \%)$ & $340(49,5 \%)$ & $21(9,7 \%)$ & $377(31,3 \%)$ & $<0,0001$ & $\begin{array}{l}\text { A-B: }<0,0001 \\
\text { A-C: } 0,062 \\
\text { B-C: }<0,0001\end{array}$ \\
\hline TKR & $15(5,0 \%)$ & $277(40,3 \%)$ & $14(6,4 \%)$ & $306(25,4 \%)$ & & \\
\hline AKR & $0(0.0 \%)$ & $12(1,7 \%)$ & $5(2,3 \%)$ & $17(1,4 \%)$ & & \\
\hline AKE & $0(0,0 \%)$ & $16(2,3 \%)$ & $1(0,5 \%)$ & $17(1,4 \%)$ & & \\
\hline Mehrfach $(\mathrm{TK}+\ldots)$ & $1(0,3 \%)$ & $35(5,1 \%)$ & $1(0,5 \%)$ & $37(3,1 \%)$ & & \\
\hline $\begin{array}{l}\text { Zusätzlicher } \\
\text { sonstiger } \\
\text { Eingriff }\end{array}$ & $13(4,3 \%)$ & $33(4,8 \%)$ & $12(5,5 \%)$ & $58(4,8 \%)$ & 0,127 & \\
\hline ASD & $13(4,3 \%)$ & $20(2,9 \%)$ & $9(4,1 \%)$ & $42(3,5 \%)$ & & \\
\hline Sonstige & $0(0,0 \%)$ & $13(1,9 \%)$ & $3(1,4 \%)$ & $16(1,3 \%)$ & & \\
\hline ACB & $2(0,7 \%)$ & $13(1,9 \%)$ & $0(0,0 \%)$ & $15(1,2 \%)$ & 0,051 & \\
\hline Ablation & $59(19,7 \%)$ & $127(18,5 \%)$ & $48(21,9 \%)$ & $234(19,4 \%)$ & 0,530 & \\
\hline Endo Kryo & $10(3,3 \%)$ & $73(10,6 \%)$ & $36(16,4 \%)$ & $119(9,9 \%)$ & & \\
\hline Endo HF & $49(16,3 \%)$ & $52(7,6 \%)$ & $12(5,5 \%)$ & $113(9,4 \%)$ & & \\
\hline $\begin{array}{l}\text { Epi Kryo } \\
\text { (Atricure Zange) }\end{array}$ & 0 & $2(0,3 \%)$ & 0 & $2(0,2 \%)$ & & \\
\hline
\end{tabular}

Tabelle 6: Mitralklappeneingriff und zusätzliche Eingriffe 


\subsubsection{Perioperative Schrittmacherimplantationen}

Bei 3,9 \% der Patienten wurde perioperativ (hier definiert als innerhalb von 14 Tagen postoperativ) eine Herzschrittmacherimplantation notwendig.

In Gruppe B und C war dies im Vergleich zu Gruppe A, in der es nur einen einzigen Fall gab, signifikant häufiger (siehe Tabelle 7).

Unter den chirurgisch abladierten Patienten (Tabelle 8) war die Notwendigkeit für eine perioperative Implantation eines Schrittmachers mit 9,4\% häufiger gegeben als in der Gruppe der nicht abladierten (Tabelle 9) mit 2,6 \%. In beiden Fällen ergab sich ein signifikanter Unterschied zwischen den drei Gruppen. Nach Ablation hatte Gruppe C den höchsten Anteil perioperativer Schrittmacherimplantationen, gefolgt von Gruppe B; in Gruppe A gab es keinen Fall. Es bestand ein signifikanter Unterschied zwischen allen drei Gruppen. Unter den nicht abladierten Patienten gab es einen signifikanten Unterschied zwischen Gruppe B mit 3,8 \% und Gruppe A mit nur einem Fall (0,3 \%) einer Schrittmacherindikation.

\begin{tabular}{|l|l|l|l|l|l|l|}
\hline & $\begin{array}{l}\text { Gruppe A } \\
\mathbf{n = 3 0 0}\end{array}$ & $\begin{array}{l}\text { Gruppe B } \\
\mathbf{n = 6 8 7}\end{array}$ & $\begin{array}{l}\text { Gruppe C } \\
\mathbf{n = 2 1 9}\end{array}$ & $\begin{array}{l}\text { Gesamt } \\
\mathbf{n = 1 2 0 6}\end{array}$ & $\begin{array}{l}\mathbf{p} \text {-Wert } \\
\text { (Globaltest) }\end{array}$ & $\begin{array}{l}\mathbf{p} \text {-Wert } \\
\text { (Paarvergleiche) }\end{array}$ \\
\hline $\begin{array}{l}\text { Perioperative } \\
\text { Schrittmacher- } \\
\text { implantation }\end{array}$ & $1(0,3 \%)$ & $31(4,5 \%)$ & $15(6,8 \%)$ & $47(3,9 \%)$ & $\mathbf{0 , 0 0 0 4}$ & $\begin{array}{l}\text { A-B: } \mathbf{0 , 0 0 2} \\
\text { A-C: }<\mathbf{0 , 0 0 1} \\
\text { B-C: } \mathbf{0 , 2 1 4}\end{array}$ \\
\hline
\end{tabular}

Tabelle 7: Perioperativ implantierte Schrittmacheranzahl

\begin{tabular}{|l|l|l|l|l|l|l|}
\hline & $\begin{array}{l}\text { Gruppe A } \\
\mathbf{n = 5 9}\end{array}$ & $\begin{array}{l}\text { Gruppe B } \\
\mathbf{n = 1 2 7}\end{array}$ & $\begin{array}{l}\text { Gruppe C } \\
\mathbf{n = 4 8}\end{array}$ & $\begin{array}{l}\text { Gesamt } \\
\mathbf{n = 2 3 4}\end{array}$ & $\begin{array}{l}\text { p-Wert } \\
\text { (Globaltest) }\end{array}$ & $\begin{array}{l}\text { p-Wert } \\
\text { (Paarvergleiche) }\end{array}$ \\
\hline $\begin{array}{l}\text { Perioperative } \\
\text { Schrittmacher- } \\
\begin{array}{l}\text { Implantation nach } \\
\text { Ablation }\end{array}\end{array}$ & $0(0 \%)$ & $10(7,9 \%)$ & $12(25,0 \%)$ & $22(9,4 \%)$ & $<\mathbf{0 , 0 0 0 1}$ & $\begin{array}{l}\text { A-B: } \mathbf{0 , 0 3 2} \\
\text { A-C: } \mathbf{0 , 0 0 1}\end{array}$ \\
\hline
\end{tabular}

Tabelle 8: Perioperativ implantierte Schrittmacheranzahl nach Ablation 


\begin{tabular}{|l|l|l|l|l|l|l|}
\hline & $\begin{array}{l}\text { Gruppe A } \\
\mathbf{n = 2 4 1}\end{array}$ & $\begin{array}{l}\text { Gruppe B } \\
\mathbf{n = 5 6 0}\end{array}$ & $\begin{array}{l}\text { Gruppe C } \\
\mathbf{n = 1 7 1}\end{array}$ & $\begin{array}{l}\text { Gesamt } \\
\mathbf{n = 9 7 2}\end{array}$ & $\begin{array}{l}\mathbf{p} \text {-Wert } \\
\text { (Globaltest) }\end{array}$ & $\begin{array}{l}\text { p-Wert } \\
\text { (Paarvergleiche) }\end{array}$ \\
\hline $\begin{array}{l}\text { Perioperative } \\
\text { Schrittmacher- } \\
\text { implantation ohne } \\
\text { Ablation }\end{array}$ & $1(0,4 \%)$ & $21(3,8 \%)$ & $3(1,8 \%)$ & $25(2,6 \%)$ & $\mathbf{0 , 0 1 6}$ & $\begin{array}{l}\text { A-B: } \mathbf{0 , 0 2 4} \\
\text { A-C: } \mathbf{0 , 4 6 4} \\
\text { B-C: } \mathbf{0 , 4 6 4}\end{array}$ \\
\hline
\end{tabular}

Tabelle 9: Perioperativ implantierte Schrittmacheranzahl der nicht abladierten Patienten

\subsubsection{Frühsterblichkeit}

Die Sterblichkeit innerhalb von 30 Tagen postoperativ war in Gruppe B prozentual höher als in den beiden anderen Gruppen. An dieser Stelle sollte daran erinnert werden, dass in Gruppe B der Anteil an zusätzlichen Klappeneingriffen höher war als in den anderen beiden Gruppen. Die erhöhte Mortalität könnte damit in Verbindung gebracht werden. Signifikant war der Unterschied in der Frühsterblichkeit zwischen den drei Gruppen nicht. Es gab zudem Patienten, die zwar außerhalb des Zeitraums von 30 Tagen postoperativ (Frühsterblichkeit), aber noch intrahospital verstarben. Unter diesen war der Anteil in Gruppe B ebenfalls etwas höher als in den anderen beiden Gruppen.

\begin{tabular}{|l|l|l|l|l|l|l|}
\hline & $\begin{array}{l}\text { Gruppe A } \\
\mathbf{n}=300\end{array}$ & $\begin{array}{l}\text { Gruppe B } \\
\mathbf{n = 6 8 7}\end{array}$ & $\begin{array}{l}\text { Gruppe C } \\
\mathbf{n = 2 1 9}\end{array}$ & $\begin{array}{l}\text { Gesamt } \\
\mathbf{n = 1 2 0 6}\end{array}$ & $\begin{array}{l}\mathbf{p} \text {-Wert } \\
\text { (Globaltest) }\end{array}$ & $\begin{array}{l}\text { p-Wert } \\
\text { (Paarvergleiche) }\end{array}$ \\
\hline Frühsterblichkeit & & & & $\mathbf{0 , 0 4 6}$ & $\begin{array}{l}\text { A-B: } \mathbf{0 , 1 2 0} \\
\text { A-C: } \mathbf{0 , 9 5 0} \\
\text { B-C: } \mathbf{0 , 2 0 2}\end{array}$ \\
\hline $\begin{array}{l}\text { intrahospital } \\
>30 \text { Tage }\end{array}$ & $2(2,3 \%)$ & $39(5,7 \%)$ & $5(2,3 \%)$ & $51(4,2 \%)$ & & \\
\hline
\end{tabular}

Tabelle 10: Frühsterblichkeit 


\subsection{Postoperative Daten (zum Zeitpunkt der Entlassung)}

In diesem Abschnitt werden die postoperativ (das bedeutet: die vor der Entlassung aus dem stationären Aufenthalt) erhobenen Daten betrachtet.

\subsubsection{Postoperativer Herzrhythmus}

In den nachfolgenden Tabellen 11-14 wird der postoperativ erhobene Herzrhythmus vor der Entlassung aus dem stationären klinischen Aufenthalt dargestellt. Dabei wird der Grundrhythmus des Herzens betrachtet, das bedeutet, in diesem Fall sind auch Patienten mit Herzschrittmacher inkludiert. Beschrieben wird zunächst der Herzrhythmus des gesamten Patientenkollektivs sowie anschließend der Untergruppen der Abladierten und der Patienten mit präoperativem Sinusrhythmus.

Insgesamt hatten bei Entlassung 23,7 \% der Patienten Vorhofflimmern, was deutlich unter dem präoperativen Wert von 39,8 \% liegt. Der größte Anteil der Patenten mit Vorhofflimmern war mit 30,4\% der Gruppe B zuzuordnen. Hier ergab sich ein signifikanter Unterschied von Gruppe B zu den Gruppen A und $\mathrm{C}$.

\begin{tabular}{|c|c|c|c|c|c|c|}
\hline & $\begin{array}{l}\text { Gruppe A } \\
n=280\end{array}$ & $\begin{array}{l}\text { Gruppe B } \\
n=649\end{array}$ & $\begin{array}{l}\text { Gruppe C } \\
n=213\end{array}$ & $\begin{array}{l}\text { Gesamt } \\
n=1142\end{array}$ & $\begin{array}{l}\text { p-Wert } \\
\text { (Globaltest) }\end{array}$ & $\begin{array}{l}\text { p-Wert } \\
\text { (Paarvergleiche) }\end{array}$ \\
\hline $\begin{array}{l}\text { Postoperativer } \\
\text { Herzrhythmus }\end{array}$ & & & & & $<0,0001$ & $\begin{array}{l}\text { A-B: }<0,001 \\
\text { A-C: } \quad 0,305 \\
\text { B-C: } \quad 0,0002\end{array}$ \\
\hline SR & $242(86,4 \%)$ & $452(69,6 \%)$ & $177(83,1 \%)$ & $871(76,3 \%)$ & & \\
\hline VHF & $38(13,6 \%)$ & 197 (30,4 \%) & $36(16,9 \%)$ & $271(23,7 \%)$ & & \\
\hline
\end{tabular}

Tabelle 11: Postoperativer Herzrhythmus bei Entlassung

Von den Patienten, die aufgrund von präoperativem Vorhofflimmern chirurgisch abladiert wurden, hatten $36,7 \%$ bei Entlassung weiterhin Vorhofflimmern. In Gruppe B schien der primäre Ablationserfolg am geringsten zu sein. Dort hatten 49,2\% der Patienten bei Entlassung weiterhin Vorhofflimmern. 
Im Vergleich dazu war der Erfolg bei Gruppe A signifikant größer, hier konnten $86,4 \%$ mit einem Sinusrhythmus entlassen werden.

\begin{tabular}{|l|l|l|l|l|l|l|}
\hline & $\begin{array}{l}\text { Gruppe A } \\
\mathbf{n = 5 3}\end{array}$ & $\begin{array}{l}\text { Gruppe B } \\
\mathbf{n = 1 2 2}\end{array}$ & $\begin{array}{l}\text { Gruppe C } \\
\mathbf{n}=\mathbf{4 6}\end{array}$ & $\begin{array}{l}\text { Gesamt } \\
\mathbf{n = 2 2 1}\end{array}$ & $\begin{array}{l}\text { p-Wert } \\
\text { (Globaltest) }\end{array}$ & $\begin{array}{l}\text { p-Wert } \\
\text { (Paarvergleiche) }\end{array}$ \\
\hline $\begin{array}{l}\text { Postoperativer } \\
\text { Herzrhythmus } \\
\text { nach Ablation }\end{array}$ & & & & & $<0,0001$ & $\begin{array}{l}\text { A-B: }<0,001 \\
\text { A-C: } 0,058 \\
\text { B-C: } \mathbf{0 , 0 5 8}\end{array}$ \\
\hline SR & $46(86,8 \%)$ & $62(50,8 \%)$ & $32(69,6 \%)$ & $140(63,3 \%)$ & & \\
\hline VHF & $7(13,2 \%)$ & $60(49,2 \%)$ & $14(30,4 \%)$ & $81(36,7 \%)$ & & \\
\hline
\end{tabular}

Tabelle 12: Postoperativer Herzrhythmus nach Ablation bei Entlassung

Bei Entlassung hatten insgesamt 9,6 \% im Vergleich zu präoperativ 5,6 \% der Patienten einen Herzschrittmacher (siehe Tabelle 15). Diese Zahl inkludiert alle Patienten, die entweder bereits präoperativ oder perioperativ mit einem Schrittmacher versorgt worden waren.

Entsprechend der erhöhten Anzahl perioperativer Schrittmacherimplantationen in Gruppe B, gefolgt von Gruppe C, wurden auch postoperativ in diesen beiden Gruppen im Vergleich zu Gruppe A signifikant mehr Herzschrittmacher verzeichnet.

\begin{tabular}{|l|l|l|l|l|l|l|}
\hline & $\begin{array}{l}\text { Gruppe A } \mathbf{n} \\
\mathbf{2} \mathbf{2 8 0}\end{array}$ & $\begin{array}{l}\text { Gruppe B } \\
\mathbf{n = 6 4 9}\end{array}$ & $\begin{array}{l}\text { Gruppe C } \mathbf{n} \\
\mathbf{2} \mathbf{2 1 3}\end{array}$ & $\begin{array}{l}\text { Gesamt } \\
\mathbf{n = 1 1 4 2}\end{array}$ & $\begin{array}{l}\mathbf{p} \text {-Wert } \\
\text { (Globaltest) }\end{array}$ & $\begin{array}{l}\mathbf{p} \text {-Wert } \\
\text { (Paarvergleiche) }\end{array}$ \\
\hline $\begin{array}{l}\text { Schrittmacher } \\
\text { postoperativ } \\
\text { gesamt }\end{array}$ & $\begin{array}{l}10(3,6 \% \\
\text { von } \mathrm{n}=280)\end{array}$ & $\begin{array}{l}80(12,2 \% \\
\text { von } \mathrm{n}=649)\end{array}$ & $\begin{array}{l}20(9,4 \% \\
\text { von } \mathrm{n}=213)\end{array}$ & $\begin{array}{l}111(9,6 \% \\
\text { von } \mathrm{n}=1142)\end{array}$ & 0,0001 & $\begin{array}{l}\text { A-B: }<\mathbf{0 , 0 0 1} \\
\text { A-C: } \mathbf{0 , 0 0 8} \\
\text { B-C: } \mathbf{0 , 3 3 1}\end{array}$ \\
\hline
\end{tabular}

Tabelle 13: Schrittmacheranzahl bei Entlassung

Unter den nicht abladierten Patienten war - unabhängig vom präoperativen Herzrhythmus in Gruppe B - im Vergleich zu den beiden anderen Gruppen ein signifikant häufigeres Vorkommen von Vorhofflimmern zu finden. 


\begin{tabular}{|l|l|l|l|l|l|l|}
\hline & $\begin{array}{l}\text { Gruppe A } \\
\mathbf{n}=227\end{array}$ & $\begin{array}{l}\text { Gruppe B } \\
\mathbf{n}=\mathbf{5 2 7}\end{array}$ & $\begin{array}{l}\text { Gruppe C } \\
\mathbf{n = 1 6 7}\end{array}$ & $\begin{array}{l}\text { Gesamt } \\
\mathbf{n = 9 2 1}\end{array}$ & $\begin{array}{l}\text { p-Wert } \\
\text { (Globaltest) }\end{array}$ & $\begin{array}{l}\text { p-Wert } \\
\text { (Paarvergleiche) }\end{array}$ \\
\hline $\begin{array}{l}\text { Postoperativer } \\
\text { Herzrhythmus } \\
\text { ohne Ablation }\end{array}$ & & & & & $<0,0001$ & $\begin{array}{l}\text { A-B: }: 0,001 \\
\text { A-C: } 0,890\end{array}$ \\
\hline SR & $196(86,3 \%)$ & $390(74,0 \%)$ & $145(86,8 \%)$ & $731(79,4 \%)$ & & \\
\hline VHF & $31(13,7 \%)$ & $137(26,0 \%)$ & $22(13,2 \%)$ & $190(20,6 \%)$ & & \\
\hline
\end{tabular}

Tabelle 14: Postoperativer Herzrhythmus der nicht abladierten Patienten bei Entlassung

Um die Anzahl von postoperativ neu aufgetretenem Vorhofflimmern zu detektieren, wurden in Tabelle 14 nur die Patienten mit präoperativem Sinusrhythmus betrachtet.

Wenngleich der prozentuale Anteil an Vorhofflimmern in den Gruppen B und C größer war als in Gruppe A, konnte rechnerisch kein signifikanter Unterschied ermittelt werden.

\begin{tabular}{|l|l|l|l|l|l|}
\hline & $\begin{array}{l}\text { Gruppe A } \\
\mathbf{n}=177\end{array}$ & $\begin{array}{l}\text { Gruppe B } \\
\mathbf{n}=375\end{array}$ & $\begin{array}{l}\text { Gruppe C } \\
\mathbf{n = 1 5 0}\end{array}$ & $\begin{array}{l}\text { Gesamt } \\
\mathbf{n = 7 0 2}\end{array}$ & $\begin{array}{l}\text { p-Wert } \\
\text { (Globaltest) }\end{array}$ \\
\hline $\begin{array}{l}\text { Postoperativer Herzrhythmus bei } \\
\text { präoperativem SR }\end{array}$ & & & & $\mathbf{0 , 1 5 9}$ \\
\hline SR & $164(92,7 \%)$ & $327(87,2 \%)$ & $134(89,0 \%)$ & $625(89,0 \%)$ & \\
\hline Vorhofflimmern & $13(7,3 \%)$ & $48(12,8 \%)$ & $16(10,7 \%)$ & $77(11,0 \%)$ & \\
\hline
\end{tabular}

Tabelle 15: Postoperativer Herzrhythmus der Patienten mit präoperativem Sinusrhythmus

\subsection{Follow-up}

\subsubsection{Teilnahme und mittlere Follow-up-Zeit}

Insgesamt konnte bei 1050 der 1141 überlebenden Patienten (92,0 \%) ein Follow-up mit Rhythmusdaten erhoben werden. Bei den fehlenden 91 Patienten war es trotz intensiver Bemühungen nicht möglich, Informationen diesbezüglich zu generieren. 
Die Gründe dafür waren mangelnde Kooperation, nicht mehr aktuelle Kontaktdaten, fehlende Befunde oder Umzug in das Ausland, wodurch die Patienten nicht mehr ausfindig gemacht werden konnten.

\begin{tabular}{|l|l|l|l|l|}
\hline & $\begin{array}{l}\text { Gruppe A } \\
\mathbf{n}=\mathbf{2 9 1}\end{array}$ & $\begin{array}{l}\text { Gruppe B } \\
\mathbf{n = 6 3 8}\end{array}$ & $\begin{array}{l}\text { Gruppe C } \\
\mathbf{n = 2 1 2}\end{array}$ & $\begin{array}{l}\text { Gesamt } \\
\mathbf{n}=\mathbf{1 1 4 1}\end{array}$ \\
\hline Follow-up-Anzahl & $257(88,2 \%)$ & $591(86,4 \%)$ & $202(95,3 \%)$ & $1050(92,0 \%)$ \\
\hline
\end{tabular}

Tabelle 16: Follow-up-Anzahl unter den Überlebenden

Die durchschnittliche Follow-up-Zeit von der Entlassung bis zum letzten Follow-up betrug $76 \pm 49$,4 Monate.

Die stark variierenden Follow-up-Zeiten entstanden aufgrund dessen, dass zu unterschiedlichen Zeitpunkten bevorzugt einer der genannten Zugangswege an der Universitätsklinik Frankfurt am Main angewandt wurde.

Die in Gruppe A verwendete anterolaterale Minithorakotomie unter Verwendung der Chitwood-Klemme wurde hauptsächlich in den Jahren 1998-2012 durchgeführt. Ab 2004 kam die partielle obere Sternotomie zum Einsatz, mit transseptalem Zugang vorwiegend in den Jahren 2004-2015 und mit transcavalem Zugang von 2004 bis 2013.

\begin{tabular}{|l|l|l|l|l|l|l|}
\hline & $\begin{array}{l}\text { Gruppe A } \\
\mathbf{n}=257\end{array}$ & $\begin{array}{l}\text { Gruppe B } \\
\mathbf{n}=591\end{array}$ & $\begin{array}{l}\text { Gruppe C } \\
\mathbf{n}=202\end{array}$ & $\begin{array}{l}\text { Gesamt } \\
\mathbf{n = 1 0 5 0}\end{array}$ & $\begin{array}{l}\text {-Wert } \\
\text { (Globaltest) }\end{array}$ & $\begin{array}{l}\text { p-Wert } \\
\text { (Paarvergleiche) }\end{array}$ \\
\hline $\begin{array}{l}\text { Follow-up-Zeit } \\
\text { (Monate) }\end{array}$ & & & & & $<0,0001$ & $\begin{array}{l}\text { A-B: }<0,001 \\
\text { A-C: }<0,001 \\
\text { B-C: }<0,001\end{array}$ \\
\hline Mittelwert \pm SD & $128,7 \pm 46,8$ & $49,4 \pm 33,0$ & $86,41 \pm 32,9$ & $76,0 \pm 49,4$ & & \\
\hline Minimum & 2 & 0 & 2 & 0 & & \\
\hline Maximum & 217 & 146 & 151 & 217 & & \\
\hline
\end{tabular}

Tabelle 17: Mittlere Follow-up-Zeit 


\subsubsection{Kumulative Follow-up-Zeit}

Die gesamte Beobachtungszeit von der Entlassung bis zum letzten Follow-up betrug 6560 Patientenjahre. In Gruppe A waren es 2696 Patientenjahre, in Gruppe B 2432 Patientenjahre und in Gruppe C 1430 Patientenjahre.

\subsubsection{Langzeitbeobachtungen}

Um Aussagen über den Langzeitherzrhythmus im Hinblick auf Vorhofflimmern oder die Notwendigkeit von Schrittmacherimplantationen in den Jahren nach der Operation machen zu können, wurden die erhobenen Daten des letzten Follow-ups eines jeden der 1050 Patienten ausgewertet.

\subsubsection{Herzrhythmus im letzten Follow-up (inkl. Grundrhythmus bei Schrittmacher)}

Auch hier wird der Grundrhythmus des Herzens betrachtet, das bedeutet, auch Patienten mit Herzschrittmacher sind inkludiert.

In der Langzeitbeobachtung waren in Gruppe B signifikant weniger Patienten im Sinusrhythmus als in den anderen beiden Gruppen. Die geringsten Vorhofflimmeranzahlen hatte Gruppe C zu verzeichnen.

\begin{tabular}{|c|c|c|c|c|c|c|}
\hline & $\begin{array}{l}\text { Gruppe A } \\
n=257\end{array}$ & $\begin{array}{l}\text { Gruppe B } \\
n=591\end{array}$ & $\begin{array}{l}\text { Gruppe C } \\
n=202\end{array}$ & $\begin{array}{l}\text { Gesamt } \\
n=1050\end{array}$ & $\begin{array}{l}\text { p-Wert } \\
\text { (Globaltest) }\end{array}$ & $\begin{array}{l}\text { p-Wert } \\
\text { (Paarvergleiche) }\end{array}$ \\
\hline $\begin{array}{l}\text { Rhythmus im } \\
\text { letzten FU }\end{array}$ & & & & & $<0,0001$ & $\begin{array}{l}\text { A-B: } \quad 0,012 \\
\text { A-C: } \quad 0,077 \\
\text { B-C: }<0,001\end{array}$ \\
\hline SR & $174(67,7 \%)$ & $341(57,7 \%)$ & $152(75,2 \%)$ & $667(63,5 \%)$ & & \\
\hline VHF & $83(32,3 \%)$ & 250 (42,3 \%) & $50(24,8 \%)$ & $383(36,5 \%)$ & & \\
\hline
\end{tabular}

Tabelle 18: Herzrhythmus im letzten Follow-up 
In Tabelle 19 wurden nur die 202 der 234 abladierten Patienten betrachtet, von denen ein Follow-up vorlag. Die Langzeitergebnisse nach Ablation zeigten einen signifikant geringeren Ablationserfolg bei Gruppe B im Vergleich zu Gruppe C.

\begin{tabular}{|l|l|l|l|l|l|l|}
\hline & $\begin{array}{l}\text { Gruppe A } \\
\mathbf{n = 5 1}\end{array}$ & $\begin{array}{l}\text { Gruppe B } \\
\mathbf{n = 1 1 2}\end{array}$ & $\begin{array}{l}\text { Gruppe C } \\
\mathbf{n}=\mathbf{3 9}\end{array}$ & $\begin{array}{l}\text { Gesamt } \\
\mathbf{n = 2 0 2}\end{array}$ & $\begin{array}{l}\text { p-Wert } \\
\text { (Globaltest) }\end{array}$ & $\begin{array}{l}\text { p-Wert } \\
\text { (Paarvergleiche) }\end{array}$ \\
\hline $\begin{array}{l}\text { Rhythmus im } \\
\text { letzten FU } \\
\text { nach Ablation }\end{array}$ & & & & & $\mathbf{0 , 0 4 4}$ & $\begin{array}{l}\text { A-B: } \mathbf{0 , 4 1 2} \\
\text { A-C: } 0,412 \\
\text { B-C: } 0,045\end{array}$ \\
\hline SR & $24(47,1 \%)$ & $41(36,6 \%)$ & $23(59,0 \%)$ & $88(43,6 \%)$ & & \\
\hline VHF & $27(52,9 \%)$ & $71(63,4 \%)$ & $16(41,0 \%)$ & $114(56,4 \%)$ & & \\
\hline
\end{tabular}

Tabelle 19: Herzrhythmus im letzten Follow-up nach Ablation

Hieraus errechnet sich eine Inzidenz für ein Vorhofflimmerrezidiv nach Ablation von 1,0 \% für Gruppe A, 2,9 \% für Gruppe B und 1,1 \% für Gruppe C pro Patientenjahr.

Für die Berechnung wurde die kumulative Follow-up-Zeit von 2696 Patientenjahren in Gruppe A, 2432 in Gruppe B und 1430 in Gruppe C verwendet.

Bei den verbleibenden nicht abladierten 848 Patienten hatten in Gruppe B signifikant mehr Vorhofflimmern als in den beiden anderen Gruppen.

\begin{tabular}{|l|l|l|l|l|l|l|}
\hline & $\begin{array}{l}\text { Gruppe A } \\
\mathrm{n}=\mathbf{2 0 6}\end{array}$ & $\begin{array}{l}\text { Gruppe B } \\
\mathrm{n}=\mathbf{4 7 9}\end{array}$ & $\begin{array}{l}\text { Gruppe C } \\
\mathrm{n=163}\end{array}$ & $\begin{array}{l}\text { Gesamt } \\
\mathbf{n = 8 4 8}\end{array}$ & $\begin{array}{l}\text { p-Wert } \\
\text { (Globaltest) }\end{array}$ & $\begin{array}{l}\text { p-Wert } \\
\text { (Paarvergleiche) }\end{array}$ \\
\hline $\begin{array}{l}\text { Rhythmus im } \\
\text { letzten FU } \\
\text { ohne Ablation }\end{array}$ & & & & $\mathbf{0 , 0 0 0 1}$ & $\begin{array}{l}\text { A-B: } \mathbf{0 , 0 2 0} \\
\mathbf{0}, \mathbf{1 6 0} \\
\text { B-C: } \mathbf{0 , 0 0 0 3}\end{array}$ \\
\hline SR & $150(72,8 \%)$ & $300(62,6 \%)$ & $129(79,1 \%)$ & $579(68,3 \%)$ & & \\
\hline VHF & $56(27,2 \%)$ & $179(37,4 \%)$ & $34(20,9 \%)$ & $269(31,7 \%)$ & & \\
\hline
\end{tabular}

Tabelle 20: Herzrhythmus der nicht abladierten Patienten im letzten Follow-up 
Tabelle 21 zeigt die Patienten, die vor der Operation im Sinusrhythmus waren. Daraus konnte der Anteil an nach der Operation neu aufgetretenem Vorhofflimmern bestimmt werden. Dieser zeigte im letzten Follow-up keinen signifikanten Unterschied zwischen den Gruppen. Prozentual war der Anteil in Gruppe B allerdings etwas höher als in den beiden anderen Gruppen.

\begin{tabular}{|l|l|l|l|l|l|}
\hline & $\begin{array}{l}\text { Gruppe A } \\
n=161\end{array}$ & $\begin{array}{l}\text { Gruppe B } \\
\mathbf{n}=345\end{array}$ & $\begin{array}{l}\text { Gruppe C } \\
\mathbf{n = 1 4 7}\end{array}$ & $\begin{array}{l}\text { Gesamt } \\
\mathbf{n = 6 5 3}\end{array}$ & $\begin{array}{l}\text { p-Wert } \\
\text { (Globaltest) }\end{array}$ \\
\hline $\begin{array}{l}\text { Rhythmus im letzten FU } \\
\text { bei präoperativem SR }\end{array}$ & & & & 0,169 \\
\hline SR & $132(82,0 \%)$ & $264(76,5 \%)$ & $122(83,0 \%)$ & $518(79,3 \%)$ & \\
\hline Vorhofflimmern & $29(18,0 \%)$ & $81(23,5 \%)$ & $25(17,0 \%)$ & $135(20,7 \%)$ & \\
\hline
\end{tabular}

Tabelle 21: Herzrhythmus der Patienten mit präoperativem Sinusrhythmus zum Zeitpunkt des letzten Follow-ups

Hieraus errechnet sich eine Inzidenz für neu aufgetretenes Vorhofflimmern von 1,0 \% für Gruppe A, 3,3 \% für Gruppe B und 1,7 \% für Gruppe C pro Patientenjahr.

Für die Berechnung wurde die kumulative Follow-up-Zeit von 2696 Patientenjahren in Gruppe A, 2432 in Gruppe B und 1430 in Gruppe C verwendet.

\subsubsection{Anteil an Vorhofflimmern im Verlauf}

In den Abbildungen 3 und 4 werden die bereits erläuterten Ergebnisse zu den verschiedenen Zeitpunkten noch einmal grafisch zusammengefasst.

Dargestellt wird die Prävalenz von Vorhofflimmern unter den Studienpatienten zu verschiedenen Zeitpunkten des Beobachtungszeitraums.

In Abbildung 3 wurden alle Patienten betrachtet, ungeachtet dessen, ob eine Ablation erfolgt war. Präoperativ war unter allen Patienten der Anteil an Vorhofflimmern in Gruppe B am größten. Postoperativ konnte ein deutlicher Rückgang der Vorhofflimmerrate beobachtet werden. 
Der sichtbare Unterschied des postoperativen Rhythmus in den Gruppen zeigte sich auch in der Auswertung signifikant. Die Patienten in Gruppe B hatten deutlich häufiger Vorhofflimmern nach der Operation als in den anderen beiden Gruppen, wobei auch die präoperative Vorhofflimmeranzahl in dieser Gruppe am höchsten war.

Im letzten Follow-up konnte eine hohe Rate an Vorhofflimmern in der Langzeitbeobachtung deutlich gemacht werden. Diese war in Gruppe B signifikant am höchsten, so dass der prozentuale Anteil an Vorhofflimmern sich nahezu dem präoperativen Status anglich.

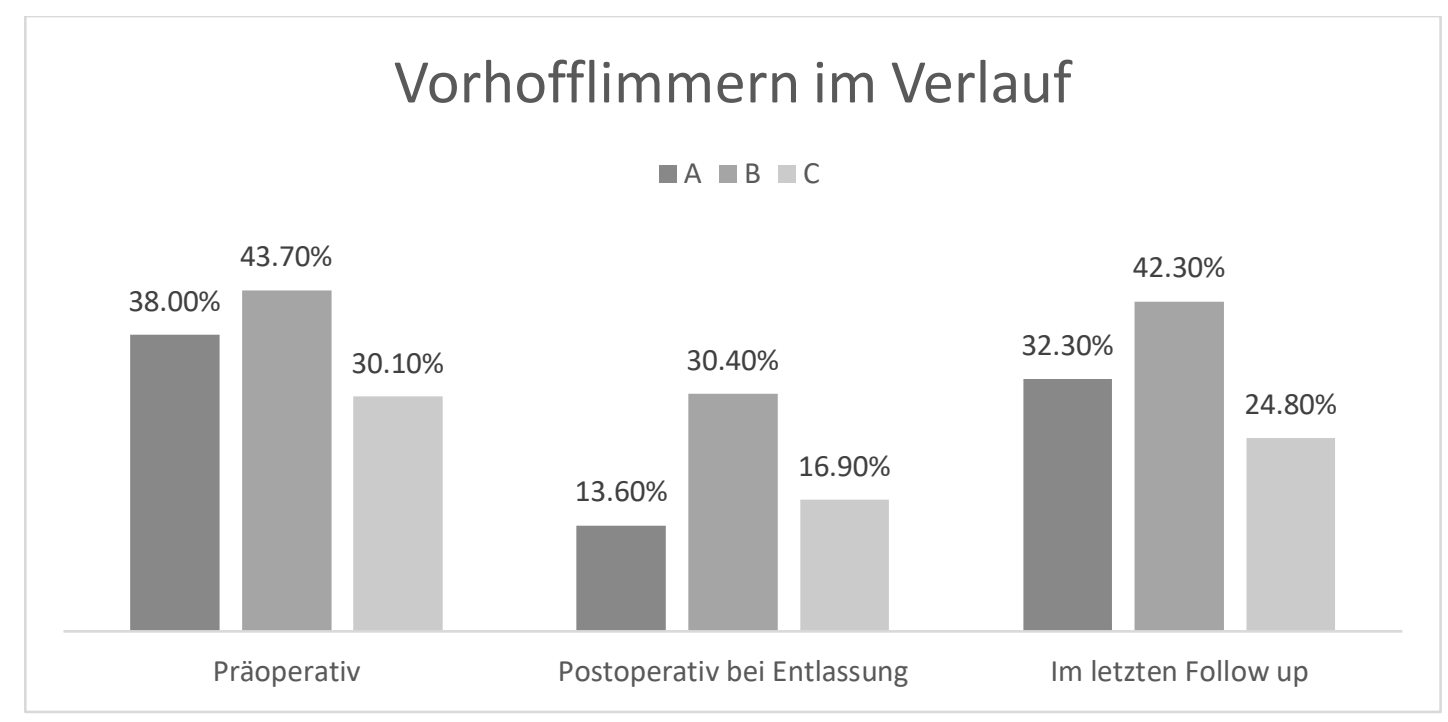

Abbildung 3: Vorhofflimmeranteil der Vergleichsgruppen im Verlauf

In Abbildung 3 wurden nur jene Patienten betrachtet, die eine chirurgische Ablation erhalten hatten. Es wurde der primäre Ablationserfolg unmittelbar nach der Operation bzw. vor der Entlassung und der Langzeiterfolg im letzten Followup dargestellt. Gruppe A wies postoperativ den größten Ablationserfolg auf. Bei diesen Patienten konnte der Vorhofflimmeranteil von $100 \%$ präoperativ auf 13,3 \% postoperativ reduziert werden. In Gruppe B konnte durch die Ablation nur bei 50,8\% der Patienten ein Sinusrhythmus erreicht werden. Dieser Unterschied wurde als signifikant errechnet. 
Im letzten Follow-up zeigte sich bei Gruppe A jedoch auch eine deutlich erhöhte Vorhofflimmerrezidivrate von 39,7 \% im Vergleich zu 14,2 \% bei Gruppe B und $10,6 \%$ bei Gruppe C.

Dabei sei noch einmal erwähnt, dass bei Gruppe A aufgrund der früheren Operationsjahre die Zeit bis zum letzten Follow-up entsprechend länger war.

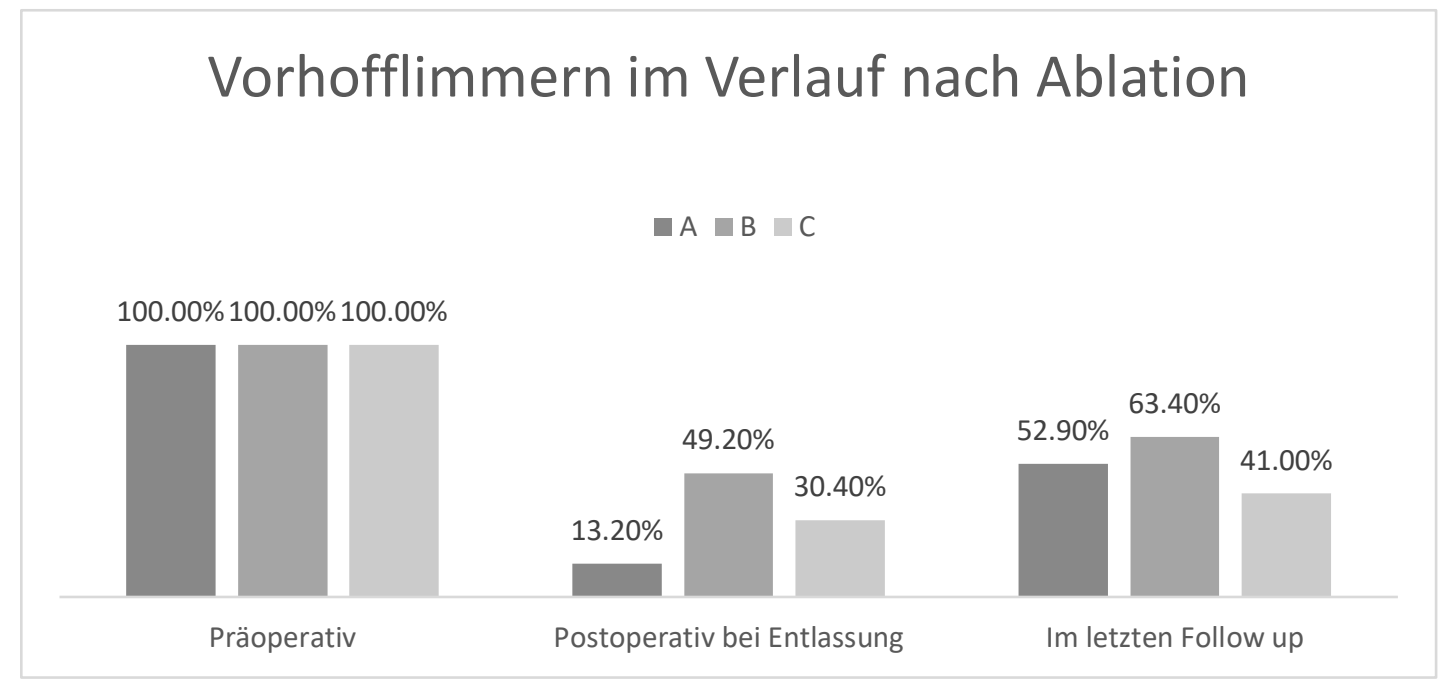

Abbildung 4: Vorhofflimmeranteil der Vergleichsgruppen nach Ablation im Verlauf

\subsubsection{Schrittmacheranzahl im letzten Follow-up}

Die folgende Tabelle zeigt die absoluten Zahlen der Patienten, die zum

Zeitpunkt des letzten Follow-ups mit einem Herzschrittmacher versorgt waren.

\begin{tabular}{|l|l|l|l|l|l|l|}
\hline & $\begin{array}{l}\text { Gruppe A } \\
\mathbf{n = 2 5 7}\end{array}$ & $\begin{array}{l}\text { Gruppe B } \\
\mathbf{n = 5 9 1}\end{array}$ & $\begin{array}{l}\text { Gruppe C } \\
\mathbf{n = 2 0 2}\end{array}$ & $\begin{array}{l}\text { Gesamt } \\
\mathbf{n = 1 0 5 0}\end{array}$ & $\begin{array}{l}\mathbf{p} \text {-Wert } \\
\text { (Globaltest) }\end{array}$ & $\begin{array}{l}\mathbf{p} \text {-Wert } \\
\text { (Paarvergleiche) }\end{array}$ \\
\hline $\begin{array}{l}\text { Schrittmacheranzahl } \\
\text { im letzten FU }\end{array}$ & $\begin{array}{l}29 \\
(11,3 \%)\end{array}$ & $\begin{array}{l}119 \\
(20,1 \%)\end{array}$ & $\begin{array}{l}39 \\
(19,3 \%)\end{array}$ & $\begin{array}{l}187 \\
(17,8 \%)\end{array}$ & $\mathbf{0 , 0 0 7}$ & $\begin{array}{l}\text { A-B: } \mathbf{0 , 0 0 6} \\
\text { A-C: } \mathbf{0 , 0 3 2} \\
\text { B-C: } \mathbf{0 , 7 9 9}\end{array}$ \\
\hline $\begin{array}{l}\text { Patienten mit nach } \\
\text { Entlassung indiziertem } \\
\text { Schrittmacher im } \\
\text { letzten FU }\end{array}$ & $\begin{array}{l}22 \\
(8,6 \%)\end{array}$ & $\begin{array}{l}76 \\
(14,6 \%)\end{array}$ & $\begin{array}{l}34 \\
(16,8 \%)\end{array}$ & $\begin{array}{l}132 \\
(12,6 \%)\end{array}$ & $\mathbf{0 , 0 2 8}$ & $\begin{array}{l}\text { A-B: } \mathbf{0 , 0 8 8} \\
\text { A-C: } \mathbf{0 , 0 2 4}\end{array}$ \\
\hline
\end{tabular}

Tabelle 22: Absolute Schrittmacherzahl zum Zeitpunkt des letzten Follow-ups 
Hieraus errechnet sich eine Inzidenz für Schrittmacherimplantationen von 0,8 \% für Gruppe A, 3,1 \% für Gruppe B und 2,3 \% für Gruppe C pro Patientenjahr. Die Berechnung erfolgte anhand der kumulativen Follow-up-Zeit von 2696 Patientenjahren in Gruppe A, 2432 in Gruppe B und 1430 in Gruppe C.

\subsubsection{Anteil an Schrittmachern im Verlauf}

Abbildung 5 stellt eine Übersicht der Schrittmacheranzahlen zu verschiedenen Zeitpunkten im Bezug zum Operationszeitpunkt dar. Daraus konnte sichtbar gemacht werden, um welchen prozentualen Anteil die Anzahl an Schrittmacherimplantationen im Verlauf gestiegen war, und somit konnte die Zahl der Neuimplantationen im jeweiligen Zeitabschnitt bestimmt werden.

Präoperativ waren die meisten der Patienten, die bereits mit einem Schrittmacher versorgt wurden, in Gruppe B zu verzeichnen.

Die postoperative Spalte stellt die Gesamtzahl der Patienten mit Schrittmacher zum Zeitpunkt der Entlassung dar, das bedeutet: präoperativ vorhandene zuzüglich perioperativ implantierte Schrittmacher. Daraus lässt sich wie beschrieben erkennen, dass die meisten perioperativen Schrittmacherimplantationen in Gruppe C mit 6,8 \% vorkamen. Diese tatsächlich geringere Anzahl im Vergleich zu den aus der Abbildung zu errechnenden $7,5 \%$ resultiert daraus, dass perioperativ einige Patienten mit implantierten Schrittmachern verstorben sind.

Bei Gruppe B waren es 4,5\% und bei Gruppe A mit nur einer einzigen Schrittmacherindikation $(0,3 \%)$ signifikant weniger als in den beiden anderen Gruppen.

Im Vergleich zum Status bei Entlassung konnte ein deutlicher Anstieg der Schrittmacheranzahl bis zum Zeitpunkt des letzten Follow-ups gezeigt werden. In Gruppe C nahm die Anzahl der Schrittmacherimplantationen in der Zeit von der Entlassung bis zum letzten Follow-up (mittlere Follow-up-Zeit 86,4 Monate \pm 32,9 SD) mit 9,5\% am deutlichsten zu. In Gruppe B zeigte sich eine Zunahme von 7,9\% (mittlere Follow-up-Zeit 49,4 Monate $\pm 33,0$ SD) und in Gruppe A 
waren es zum Zeitpunkt des letzten Follow-ups 7,7 \% mehr Schrittmacher als zum Zeitpunkt der Entlassung (mittlere Follow-up-Zeit 128,7 Monate $\pm 46,8$ $\mathrm{SD})$.

In Bezug auf die Notwendigkeit von Schrittmacherimplantationen scheint Gruppe A überlegen, da sowohl die peri- und postoperative Anzahl an Schrittmacherimplantationen als auch die Anzahl im letzten Follow-up am geringsten ist.

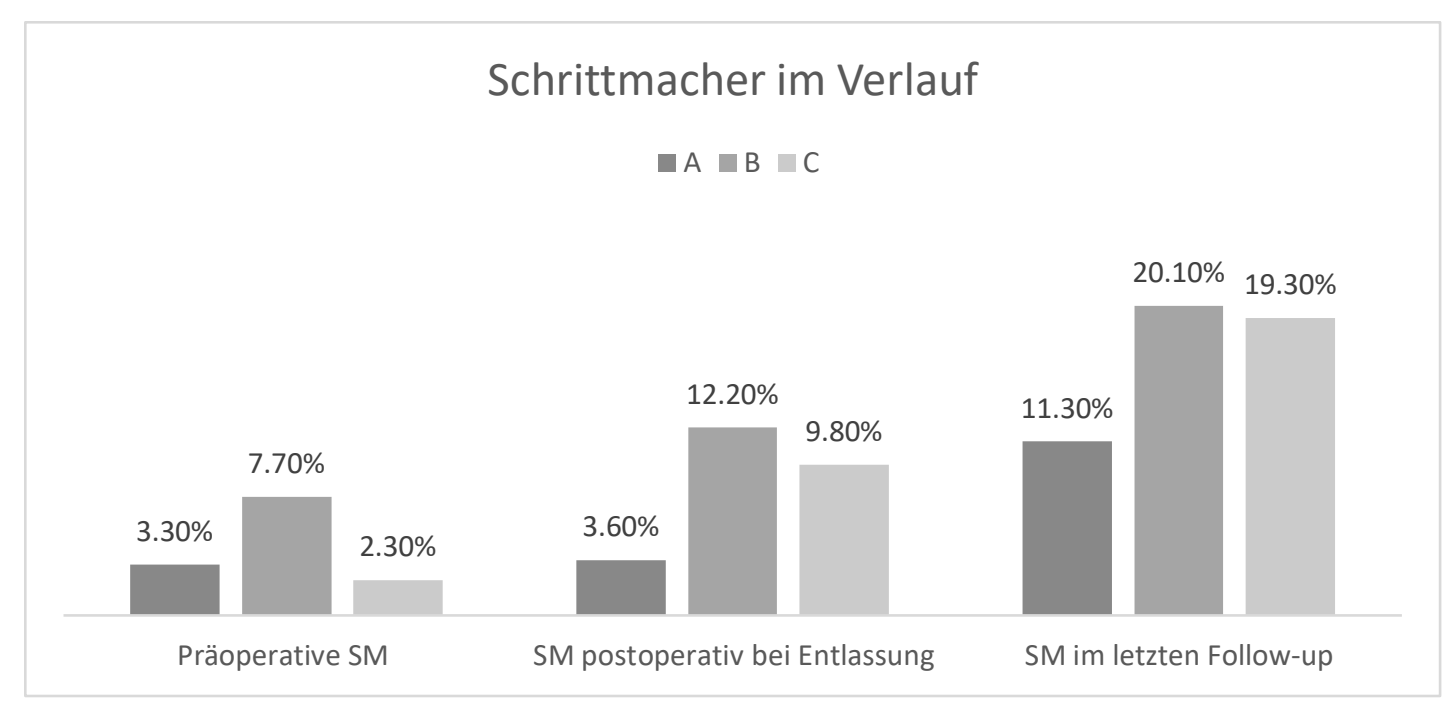

Abbildung 5: Schrittmacheranteil der Vergleichsgruppen im Verlauf

\subsection{Ereignisanzahl Schrittmacher und Defibrillatorimplantationen}

Als Ereignis wurde hier ein nach der Operation, also im Zeitraum perioperativ bis zum letzten Follow-up, implantierter Schrittmacher oder Defibrillator definiert. Die Zeitspanne reichte dabei jeweils vom Datum der Operation bis zum Implantationsdatum oder dem Zeitpunkt des letzten Kontaktes.

Es ergab sich ein signifikanter Unterschied bei der Schrittmacherzahl zwischen Gruppe A und C, wobei Erstere die geringsten und Letztere die höchsten Implantationszahlen hatte. 


\begin{tabular}{|l|l|l|l|l|l|l|}
\hline & $\begin{array}{l}\text { Gruppe A } \\
\mathbf{n = 3 0 0}\end{array}$ & $\begin{array}{l}\text { Gruppe B } \\
\mathbf{n = 6 8 7}\end{array}$ & $\begin{array}{l}\text { Gruppe C } \\
\mathbf{n = 2 1 9}\end{array}$ & $\begin{array}{l}\text { Gesamt } \\
\mathbf{n = 1 2 0 6}\end{array}$ & $\begin{array}{l}\text { p-Wert } \\
\text { (Globaltest) }\end{array}$ & $\begin{array}{l}\text { p-Wert } \\
\text { (Paarvergleiche) }\end{array}$ \\
\hline $\begin{array}{l}\text { SM-Implantationen } \\
\text { gesamt }\end{array}$ & $\begin{array}{l}22 \\
(7,3 \%)\end{array}$ & $\begin{array}{l}83 \\
(12,1 \%)\end{array}$ & $\begin{array}{l}35 \\
(16,0 \%)\end{array}$ & $\begin{array}{l}140 \\
(11,6 \%)\end{array}$ & $\mathbf{0 , 0 0 8}$ & $\begin{array}{l}\text { A-B: } \mathbf{0 , 0 5 2} \\
\text { A-C: } \mathbf{0 , 0 0 6} \\
\text { B-C: } \mathbf{0 , 1 3 5}\end{array}$ \\
\hline Nach Ablation & $8(2,7 \%)$ & $23(3,3 \%)$ & $16(7,3 \%)$ & $47(3,9 \%)$ & & \\
\hline $\begin{array}{l}\text { Defi-Implantationen } \\
\text { gesamt }\end{array}$ & $4(1,3 \%)$ & $14(2,0 \%)$ & $4(1,8 \%)$ & $22(1,8 \%)$ & $\mathbf{0 , 7 6 7}$ & \\
\hline rhythmusinduziert & $1(0,3 \%)$ & $3(0,4 \%)$ & $4(1,8 \%)$ & $8(0,7 \%)$ & & \\
\hline $\begin{array}{l}\text { keine } \\
\text { Rhythmusindikation }\end{array}$ & $3(1,0 \%)$ & $11(1,6 \%)$ & $0(0 \%)$ & $14(1,2 \%)$ & & \\
\hline
\end{tabular}

Tabelle 23: Ereignisanzahl Schrittmacher- und Defibrillatorimplantationen

\subsection{Kaplan-Meier-Schätzung der Schrittmacherhäufigkeit}

Insgesamt mussten 140 Patienten während des Beobachtungszeitraums mit einem Schrittmacher versorgt werden. Im Vergleich zur Gruppe A, in der perioperativ nur ein Schrittmacher implantiert wurde, war die Zahl bei Gruppe B und $\mathrm{C}$ deutlich höher. Auch in der Langzeitbeobachtung blieb die Zahl der Schrittmacher bei Gruppe A signifikant niedriger.

Nach 5 Jahren waren bei Gruppe A noch $98 \pm 1 \%$ der Patienten ohne Schrittmacher. In Gruppe B waren es $84 \pm 2 \%$, in Gruppe C $87 \pm 2 \%$.

Nach 10 Jahren waren es in Gruppe A immer noch $93 \pm 2 \%$, in Gruppe B $80 \pm$ $2 \%$ und in Gruppe C $78 \pm 3 \%$. 


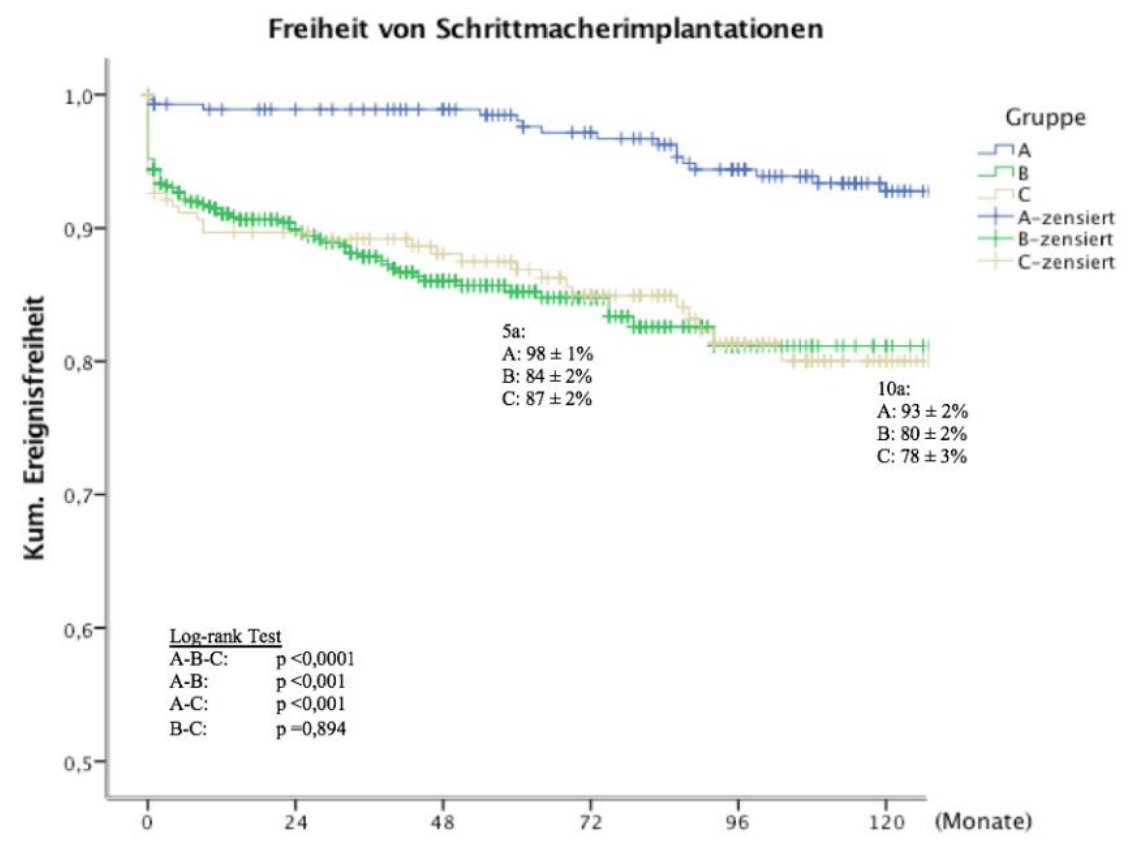

Patienten unter Risiko ( $\mathrm{n}$ )

$\begin{array}{llllccc}\text { Gruppe A } & 277 & 245 & 231 & 210 & 176 & 123 \\ \text { Gruppe B } & 561 & 324 & 203 & 99 & 32 & 9 \\ \text { Gruppe C } & 211 & 169 & 143 & 104 & 35 & 17\end{array}$

Abbildung 6: Kaplan-Meier-Kurve: Freiheit von Schrittmacherimplantationen

\begin{tabular}{|l|l|l|l|}
\hline Zeit in Monaten & \multicolumn{3}{|l|}{ Kumulierter Anteil der Schrittmacherfreien \pm SE in \% } \\
\hline & Gruppe A & Gruppe B & Gruppe C \\
\hline 24 & $99,0 \pm 1,0$ & $89 \pm 1,0$ & $90 \pm 2,0$ \\
\hline 48 & $99,0 \pm 1,0$ & $85 \pm 2,0$ & $88 \pm 2,0$ \\
\hline 72 & $97,0 \pm 1,0$ & $84 \pm 2,0$ & $85 \pm 3,0$ \\
\hline 96 & $94,0 \pm 2,0$ & $80 \pm 2,0$ & $82 \pm 3,0$ \\
\hline 120 & $93,0 \pm 2,0$ & $80 \pm 2,0$ & $80 \pm 3,0$ \\
\hline
\end{tabular}

Tabelle 24: Kumulative Schrittmacherfreiheit 
6.5.5.1.3 Kaplan-Meier-Schätzung der Defibrillatorhäufigkeit

Bei 22 Patienten wurde im Beobachtungszeitraum ein Defibrillator implantiert. Es zeigte sich kein signifikanter Unterschied zwischen den Gruppen.

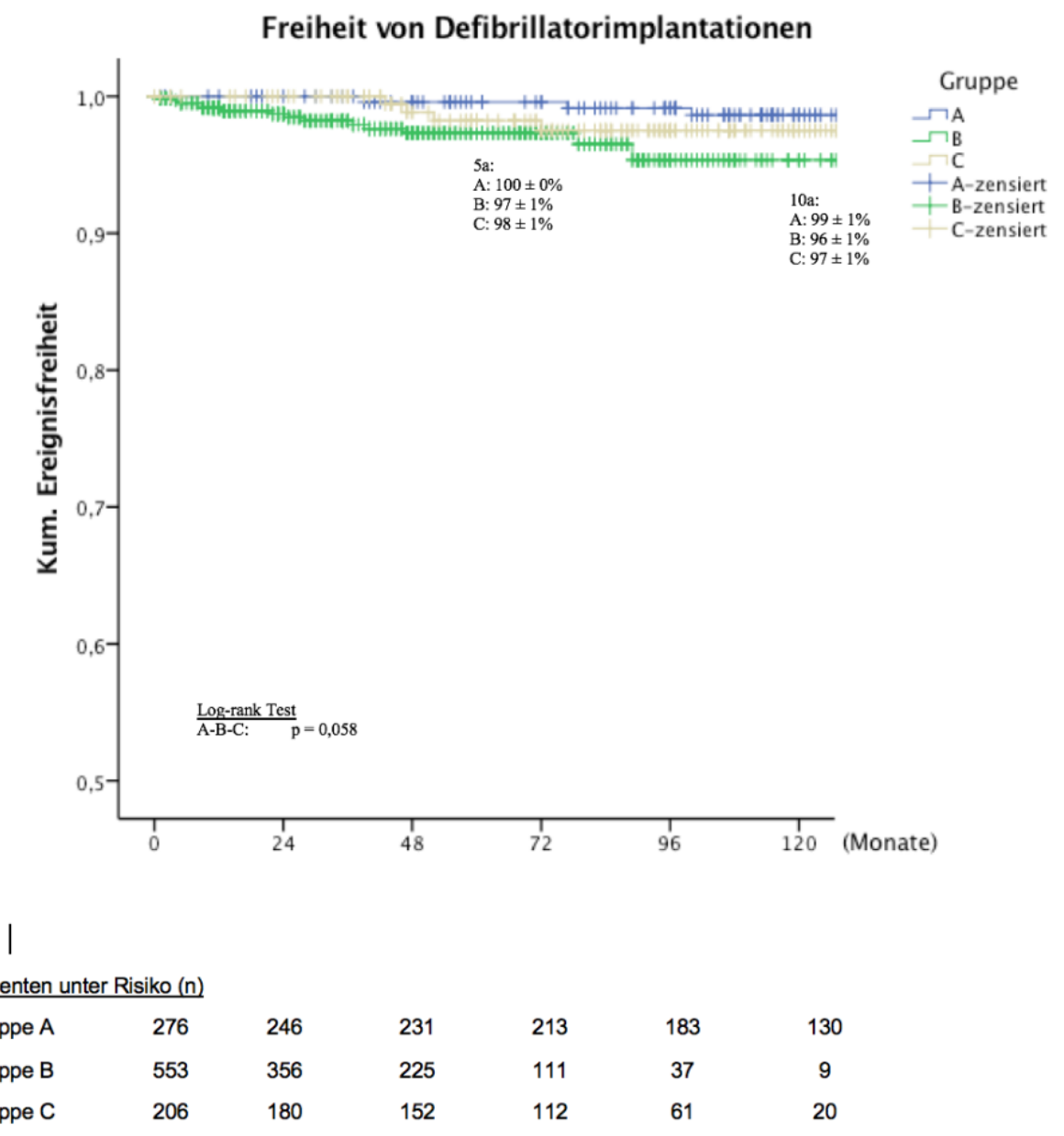

Abbildung 7: Kaplan-Meier-Kurve: Freiheit von Defibrillatorimplantationen 


\begin{tabular}{|l|l|l|l|}
\hline Zeit in Jahren & \multicolumn{3}{|l|}{ Kumulierter Anteil der Defibrillatorfreien \pm SE in \% } \\
\hline & Gruppe A & Gruppe B & Gruppe C \\
\hline 24 & $100 \pm 0$ & $99 \pm 0$ & $100 \pm 0$ \\
\hline 48 & $100 \pm 0$ & $97 \pm 1$ & $99 \pm 1$ \\
\hline 72 & $100 \pm 0$ & $97 \pm 1$ & $98 \pm 1$ \\
\hline 96 & $99 \pm 1$ & $96 \pm 1$ & $97 \pm 1$ \\
\hline 120 & $99 \pm 1$ & $96 \pm 1$ & $97 \pm 1$ \\
\hline
\end{tabular}

Tabelle 25: Kumulative Defibrillatorfreiheit

\subsection{6 Überleben}

Die Information über den Lebensstatus (verstorben/lebend) zum Zeitpunkt des individuell letzten Kontaktes lag bei allen Patienten vor. Bei Patienten mit fehlendem Follow-up wurde das Entlassdatum als letzter Kontakt gewertet. Insgesamt verstarben 257 der 1206 Patienten im Beobachtungszeitraum. Davon waren 65 Patienten bereits innerhalb von 30 Tagen nach Klinikaufenthalt oder innerhalb des stationären Aufenthaltes verstorben. Patienten, bei denen kein Todesdatum ermittelt werden konnte, wurden bis zum letzten Kontakt als lebend gewertet. Zum Zeitpunkt des letzten Follow-ups - bei einer mittleren Follow-up-Zeit von 128,7 Monaten $\pm 46,8 \mathrm{SD}$ - waren in Gruppe A noch 76,7 \% der ursprünglichen Patienten am Leben. In Gruppe B waren es 78,6 \% nach einer Zeit von 49,4 Monaten $\pm 33,0$ SD und in Gruppe C $81,7 \%$ bei 86,4 Monaten $\pm 32,9$ SD mittlere Follow-up-Zeit.

Den Überlebensstatus beim letzten Follow-up betreffend gab es keinen signifikanten Unterschied zwischen den drei Gruppen.

\begin{tabular}{|l|l|l|l|l|l|}
\hline & $\begin{array}{l}\text { Gruppe A } \\
\mathbf{n = 3 0 0}\end{array}$ & $\begin{array}{l}\text { Gruppe B } \\
\mathbf{n = 6 8 7}\end{array}$ & $\begin{array}{l}\text { Gruppe C } \\
\mathbf{n = 2 1 9}\end{array}$ & $\begin{array}{l}\text { Gesamt } \\
\mathbf{n = 1 2 0 6}\end{array}$ & $\begin{array}{l}\mathbf{p} \text {-Wert } \\
\text { (Globaltest) }\end{array}$ \\
\hline $\begin{array}{l}\text { Überlebensstatus im letzten } \\
\text { Follow-up }\end{array}$ & & & & $\mathbf{0 , 3 7 8}$ \\
\hline lebend & $230(76,7 \%)$ & $540(78,6 \%)$ & $179(81,7 \%)$ & $949(78,7 \%)$ & \\
\hline verstorben & $70(23,3 \%)$ & $147(21,4 \%)$ & $40(18,3 \%)$ & $257(21,3 \%)$ & \\
\hline
\end{tabular}

Tabelle 26: Überlebensstatus im letzten Follow-up 


\subsubsection{Kaplan-Meier-Schätzung zum Überleben}

Die folgende Kaplan-Meier-Kurve gibt Auskunft über Unterschiede der verschiedenen Gruppen im Überleben. Die Zeitspanne reichte dabei jeweils vom Datum der Operation bis zum Todesdatum.

Gruppe B schnitt hier signifikant schlechter ab, mit einem 5-Jahres-Überleben von $78 \%$ im Vergleich zu den etwa $90 \%$ der beiden anderen Gruppen. Es ist jedoch zu beachten, dass diese Gruppe auch jene mit den meisten zusätzlichen Klappeneingriffen ist.
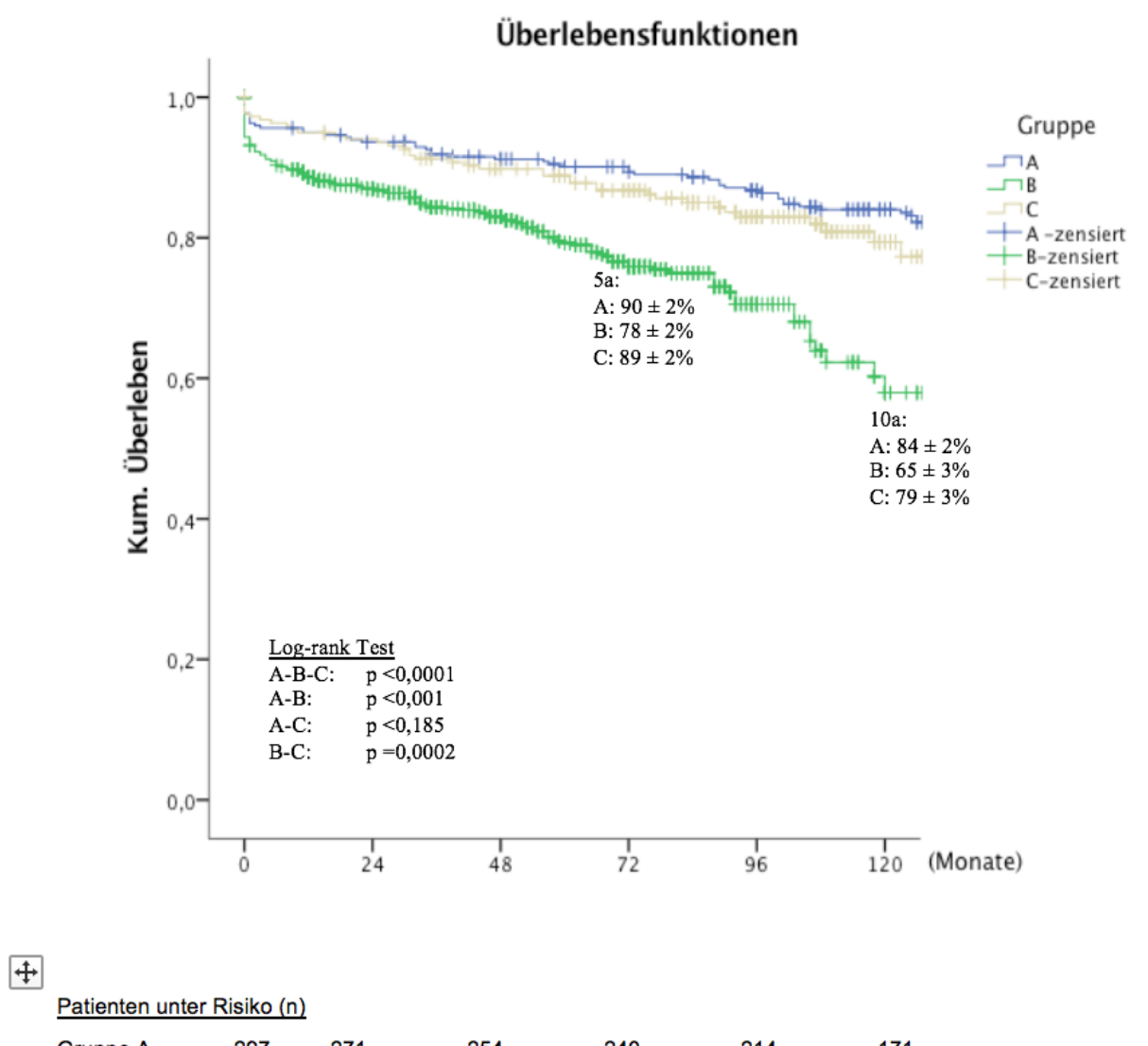

\begin{tabular}{lllllll}
\hline Gruppe A & 297 & 271 & 254 & 240 & 214 & 171 \\
Gruppe B & 624 & 421 & 290 & 146 & 53 & 16 \\
Gruppe C & 218 & 198 & 175 & 138 & 80 & 30
\end{tabular}

Abbildung 8: Kaplan-Meier-Kurve: Überleben 


\begin{tabular}{|l|l|l|l|}
\hline Zeit in Monaten & \multicolumn{4}{l|}{ Kumulierter Anteil der Überlebenden \pm SE in \% } \\
\hline & Gruppe A & Gruppe B & Gruppe C \\
\hline 24 & $94 \pm 1$ & $86 \pm 1$ & $94 \pm 2$ \\
\hline 48 & $91 \pm 2$ & $82 \pm 2$ & $90 \pm 2$ \\
\hline 72 & $90 \pm 2$ & $76 \pm 2$ & $87 \pm 2$ \\
\hline 96 & $87 \pm 2$ & $71 \pm 2$ & $83 \pm 3$ \\
\hline 120 & $84 \pm 2$ & $61 \pm 4$ & $80 \pm 3$ \\
\hline
\end{tabular}

Tabelle 27: Kumulierter Anteil Überlebender 


\section{Vergleichspopulation}

Das Überleben der Studienpatienten jeder Gruppe wurde in dieser Auswertung dem einer Vergleichspopulation aus der normalen Bevölkerung gegenübergestellt. Jedem Studienteilnehmer wurde dabei ein in Alter und Geschlecht übereinstimmender Mensch der Bevölkerung zugeteilt. In allen drei Gruppen wurde die im Vergleich zur Normalbevölkerung um etwa 5 bis $10 \%$ erhöhte Sterblichkeit maßgeblich durch die Frühsterblichkeit perioperativ entschieden. In der Nachbeobachtungszeit verlaufen die Kaplan-Meier-Kurven weitestgehend parallel.

\section{1 Überleben von Gruppe A versus Vergleichspopulation}

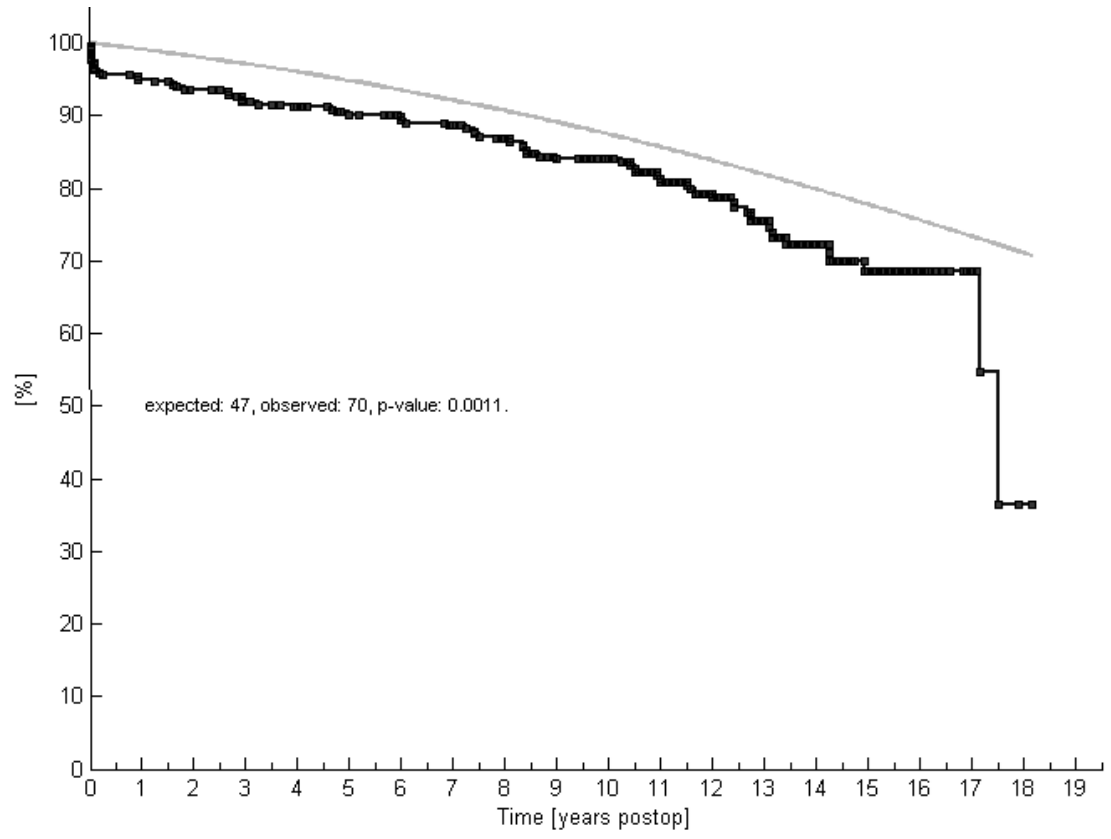

Abbildung 9: Überleben Gruppe A vs. Vergleichspopulation 


\section{2 Überleben von Gruppe B versus Vergleichspopulation}

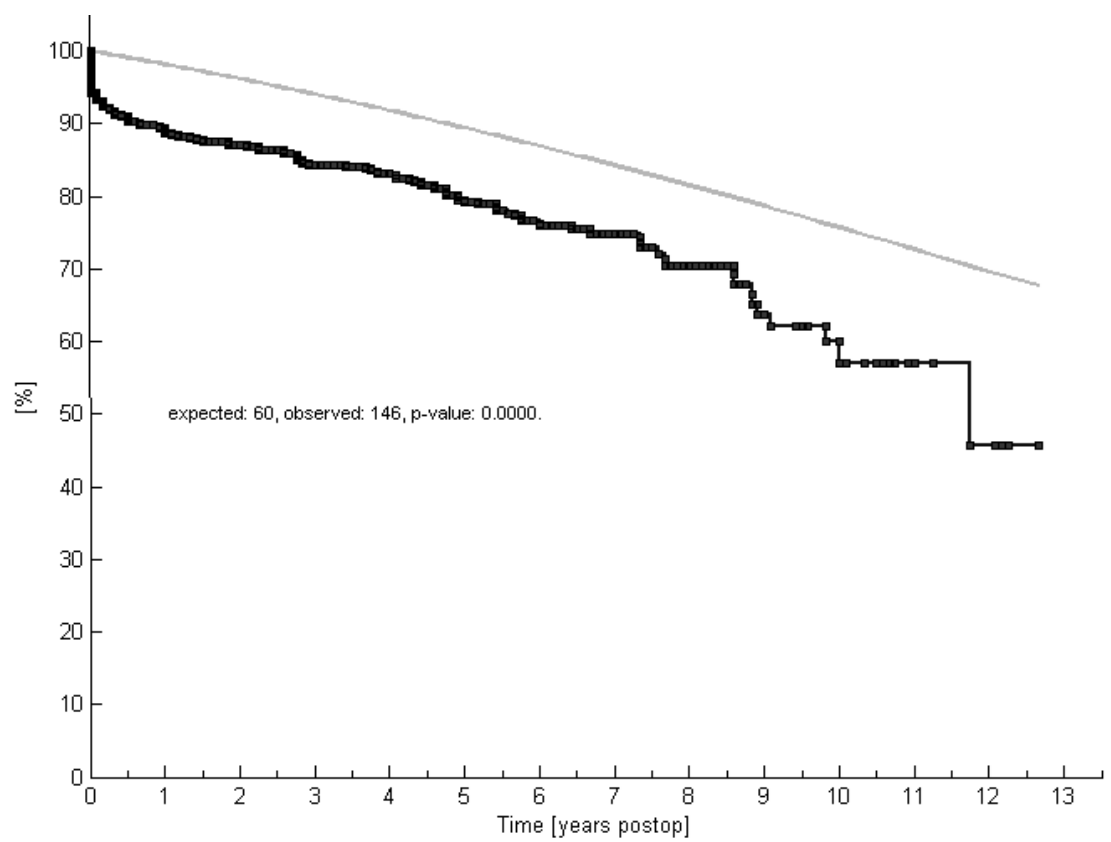

Abbildung 10: Überleben Gruppe B vs. Vergleichspopulation

\section{3 Überleben von Gruppe C versus Vergleichspopulation}

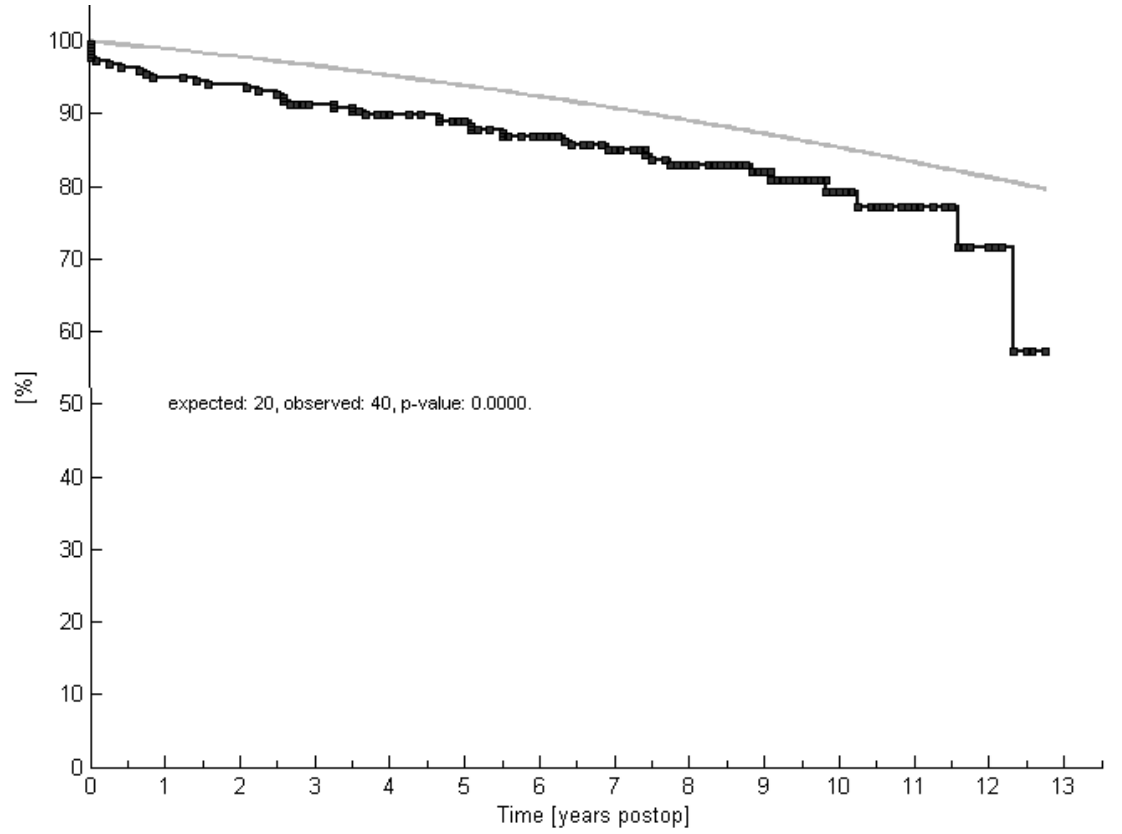

Abbildung 11: Überleben Gruppe C vs. Vergleichspopulation 


\section{Multivariate Analysen}

Es wurden multivariate Analysen zum Einfluss verschiedener Variablen auf den Ablationserfolg (logistische Regression) und auf die Anzahl der SchrittmacherImplantationen pro Zeit (Cox-Regression) durchgeführt.

Folgende Variablen wurden dabei berücksichtigt:

- Zugang

- Alter

- Pulmonaler Hypertonus

- LA-Größe präoperativ

- Chirurgische Ablation (nur bei Cox-Regression)

Bei der Variablen Zugang wurde der Zugang B (= POS transseptal) als Referenz festgelegt und der Einfluss von Zugang A (Chitwood) und Zugang $C$ (POS transcaval) im Vergleich zu dieser betrachtet.

\subsection{Logistische Regression: Ablationserfolg bei Entlassung und im letzten Follow-up}

Als Ablationserfolg wurde ein Sinusrhythmus nach erfolgter Ablation gewertet. In der Auswertung wurde Vorhofflimmern als unabhängige Variable festgelegt. Ein $\mathrm{OR}<1$ verringerte also das Risiko für Vorhofflimmern, ein $\mathrm{OR}=0$ hatte keinen Einfluss und ein OR > 1 erhöhte das Risiko für Vorhofflimmern nach Ablation.

Bei Verwendung des Chitwood-Zugangs war das Risiko für Vorhofflimmern im Vergleich zu POS-transseptal signifikant kleiner, der Ablationserfolg also größer, wobei zwischen dem POS-transseptalen und POS-transcavalen Zugang kein signifikanter Unterschied im Hinblick auf den Ablationserfolg gezeigt werden konnte.

Einen außerdem signifikanten Einfluss auf den Ablationserfolg hatten das Alter und die präoperative linksatriale Vorhofgröße. 
Je höher das Alter (in Jahren), desto größer das Risiko für Vorhofflimmern und desto geringer der Ablationserfolg.

Ein präoperativ bestehender pulmonaler Hypertonus hatte keinen Einfluss auf den Ablationserfolg.

Ein knapp signifikanter Faktor war die Vorhofgröße, die in der postoperativen, nicht aber in der Langzeitbetrachtung bei großem Durchmesser ein geringeres Risiko für Vorhofflimmern darstellte.

\subsubsection{Logistische Regression: Ablationserfolg bei Entlassung}

\begin{tabular}{|l|l|l|l|l|}
\hline Kovariaten & $\mathbf{p}$-Wert & \multicolumn{2}{l|}{ Odds Ratio (OR) } & \multicolumn{2}{l|}{$\mathbf{9 5 , 0} \%$-Konfidenzintervall } \\
\hline Zugang A vs. Zugang B & 0,042 & 0,306 & 0,098 & 0,958 \\
\hline Zugang C vs. Zugang B & 0,517 & 0,7 & 0,239 & 2,057 \\
\hline Alter & 0,006 & 1,06 & 1,017 & 1,104 \\
\hline Pulmonaler Hypertonus & 0,207 & 1,735 & 0,737 & 4,083 \\
\hline LA-Größe & 0,045 & 0,638 & 0,411 & 0,99 \\
\hline
\end{tabular}

Tabelle 28: Logistische Regression: Einflussfaktoren auf den Ablationserfolg bei Entlassung

In der Langzeitbeobachtung hatte Gruppe A im Vergleich zu Gruppe B ein geringeres Risiko für Vorhofflimmern. Mit höherem Alter stieg das Risiko für Vorhofflimmern weiterhin an. Im letzten Follow-up war ein großer linker Vorhof mit einem höheren Risiko für Vorhofflimmern assoziiert.

\subsubsection{Logistische Regression: Ablationserfolg im Last Follow-up}

\begin{tabular}{|l|l|l|l|}
\hline Kovariaten & $\mathbf{p}$-Wert & Odds Ratio (OR) & $\mathbf{9 5 , 0} \%$-Konfidenzintervall \\
\hline Zugang A vs. Zugang B & 0,71 & 0,837 & $0,328-2,137$ \\
\hline Zugang C vs. Zugang B & 0,007 & 0,185 & $0,054-0,632$ \\
\hline Alter & 0,006 & 1,054 & $1,015-1,095$ \\
\hline Pulmonaler Hypertonus & 0,394 & 0,7 & $0,308-1,591$ \\
\hline LA-Größe & 0,008 & 1,764 & $1,163-2,675$ \\
\hline
\end{tabular}

Tabelle 29: : Logistische Regression: Einflussfaktoren auf den Ablationserfolg im letzten Follow-up 


\subsection{Cox-Regression: Einfluss auf die Schrittmacherhäufigkeit}

Der Zugang über die rechtsseitige anterolaterale Minithorakotomie unter Verwendung der Chitwood-Klemme zeigte einen positiven Einfluss auf die Anzahl notwendiger Schrittmacherimplantationen im Beobachtungszeitraum.

Die partielle obere Sternotomie mit transcavalem Zugang hingegen erhöhte die Anzahl an Schrittmacherimplantationen. Das Alter und eine perioperativ durchgeführte chirurgische Ablation erhöhten das Risiko für eine spätere Schrittmacherimplantation deutlich.

\begin{tabular}{|l|l|l|l|l|}
\hline Kovariaten & $\mathbf{p}$-Wert & Hazard Ratio (HR) & \multicolumn{2}{l|}{ 95,0 \%-Konfidenzintervall } \\
\hline Zugang A vs. Zugang B & 0,025 & 0,407 & 0,186 & 0,892 \\
\hline Zugang C vs. Zugang B & 0,02 & 1,89 & 1,105 & 3,23 \\
\hline Alter & $<0,0001$ & 1,058 & 1,035 & 1,082 \\
\hline Pulmonaler Hypertonus & 0,808 & 1,061 & 0,658 & 1,712 \\
\hline LA-Größe & 0,536 & 0,925 & 0,722 & 1,184 \\
\hline Ablation & 0,00002 & 2,691 & 1,696 & 4,27 \\
\hline
\end{tabular}

Tabelle 30: Cox-Regression: Einfluss auf die Schrittmacherhäufigkeit 


\section{Matching}

Aufgrund signifikanter Unterschiede in den präoperativen Merkmalen des Patientenkollektivs mit 1206 Patienten wurde ein Matching durchgeführt.

Dieses erfolgte im Hinblick auf die folgenden wichtigen Einflussfaktoren:

- Alter

- Pulmonaler Hypertonus

- Präoperative linksatriale Vorhofgröße

- Zusätzliche Klappeneingriffe

\subsection{Methoden}

Es erfolgte ein Matching hinsichtlich der o. g. Faktoren. Aufgrund der Tatsache, dass in der vorliegenden Arbeit drei Gruppen zu vergleichen sind, erfolgte das Matching zunächst zwischen Gruppe A und B. Übrig blieben hier jeweils 284 Patienten. Anschließend wurde die gematchte Gruppe A ein zweites Mal mit der dritten Gruppe $C$ gematcht. Hieraus entsteht die deutlich höhere Fallzahl der Gruppe B, die angesichts der beinahe doppelten Größe der ursprünglichen Gruppe B jedoch kein Problem hinsichtlich der Auswertung darstellt. 


\section{Ergebnisse nach Matching}

\subsection{Patientenkollektiv}

Das Patientenkollektiv nach Matching bestand aus 700 Patienten, die im Hinblick auf die genannten Merkmale keine signifikanten Unterschiede mehr zeigten.

Mit diesem Patientenkollektiv wurden die relevanten Analysen erneut durchgeführt, um vorher gewonnene signifikante Ergebnisse zu verifizieren.

\begin{tabular}{|c|c|c|c|c|c|}
\hline & $\begin{array}{l}\text { Gruppe A } \\
n=213\end{array}$ & $\begin{array}{l}\text { Gruppe B } \\
n=274\end{array}$ & $\begin{array}{l}\text { Gruppe C } \\
n=213\end{array}$ & $\begin{array}{l}\text { Gesamt } \\
n=700\end{array}$ & $\begin{array}{l}\text { p-Wert } \\
\text { (Globaltest) }\end{array}$ \\
\hline Alter (Jahre) & & & & & 0,960 \\
\hline Mittelwert \pm SD & $56 \pm 13$ & $57 \pm 12$ & $56 \pm 13$ & $56 \pm 12$ & \\
\hline Minimum & 22 & 22 & 18 & 18 & \\
\hline Maximum & 81 & 87 & 82 & 87 & \\
\hline Pulmonaler Hypertonus & & & & & 0,157 \\
\hline gering & $40(18,8 \%)$ & $61(22,3 \%)$ & $33(15,5 \%)$ & $134(19,1 \%)$ & \\
\hline mittel & $16(7,5 \%)$ & $27(9,9 \%)$ & $21(9,9 \%)$ & $64(9,1 \%)$ & \\
\hline hochgradig & $8(3,8 \%)$ & $15(5,5 \%)$ & $11(5,2 \%)$ & $34(4,9 \%)$ & \\
\hline LA-Größe (mm) & & & & & 0,176 \\
\hline Mittelwert \pm SD & $\begin{array}{l}49,7 \pm 9,8 \\
(n=131)\end{array}$ & $\begin{array}{l}48,1 \pm 7,7 \\
(n=173)\end{array}$ & $\begin{array}{l}49,7 \pm 9,0 \\
(n=124)\end{array}$ & $\begin{array}{l}49,2 \pm 8,7 \\
(n=428)\end{array}$ & \\
\hline Zusätzlicher Klappeneingriff & & & & & 0,154 \\
\hline
\end{tabular}

Tabelle 31: Zielvariablen des Matchings 


\subsection{Präoperative Daten}

Es gab keinen signifikanten Unterschied im Patientenkollektiv hinsichtlich des präoperativen Herzrhythmus oder der Schrittmacheranzahl der jeweiligen Gruppe.

\begin{tabular}{|l|l|l|l|l|l|}
\hline & $\begin{array}{l}\text { Gruppe A } \\
\mathbf{n = 2 1 3}\end{array}$ & $\begin{array}{l}\text { Gruppe B } \\
\mathbf{n = 2 7 4}\end{array}$ & $\begin{array}{l}\text { Gruppe C } \\
\mathbf{n = 2 1 3}\end{array}$ & $\begin{array}{l}\text { Gesamt } \\
\mathbf{n = 7 0 0}\end{array}$ & $\begin{array}{l}\mathbf{p} \text {-Wert } \\
\text { (Globaltest) }\end{array}$ \\
\hline Präoperativer Rhythmus & & & & & $\mathbf{0 , 0 6 3}$ \\
\hline SR & $140(65,7 \%)$ & $208(75,9 \%)$ & $148(69,5 \%)$ & $496(70,9 \%)$ & \\
\hline VHF & $73(34,7 \%)$ & $66(24,1 \%)$ & $65(30,5 \%)$ & $204(29,1 \%)$ & \\
\hline
\end{tabular}

Tabelle 32: Präoperativer Herzrhythmus (nach Matching)

\begin{tabular}{|l|l|l|l|l|l|}
\hline & $\begin{array}{l}\text { Gruppe A } \\
\mathbf{n = 2 1 3}\end{array}$ & $\begin{array}{l}\text { Gruppe B } \\
\mathbf{n = 2 7 4}\end{array}$ & $\begin{array}{l}\text { Gruppe C } \\
\mathbf{n = 2 1 3}\end{array}$ & $\begin{array}{l}\text { Gesamt } \mathbf{n}= \\
\mathbf{7 0 0}\end{array}$ & $\begin{array}{l}\mathbf{p} \text {-Wert } \\
\text { (Globaltest) }\end{array}$ \\
\hline Schrittmacher präoperativ & $8(3,8 \%)$ & $15(5,5 \%)$ & $5(2,3 \%)$ & $28(4,0 \%)$ & $\mathbf{0 , 2 1 2}$ \\
\hline
\end{tabular}

Tabelle 33: Patienten mit präoperativem Herzschrittmacher (nach Matching)

\subsection{Perioperative Daten}

In der Häufigkeit der Ablation gab es nach dem Matching einen signifikanten Unterschied zwischen den Gruppen. In Gruppe B wurde bei einer deutlich geringeren Patientenanzahl eine chirurgische Ablation durchgeführt als in den beiden anderen Gruppen, wobei sie in Gruppe $\mathrm{C}$ am häufigsten vorkam.

\begin{tabular}{|l|l|l|l|l|l|l|}
\hline & $\begin{array}{l}\text { Gruppe A } \\
\mathbf{n = 2 1 3}\end{array}$ & $\begin{array}{l}\text { Gruppe B } \\
\mathbf{n = 2 7 4}\end{array}$ & $\begin{array}{l}\text { Gruppe C } \\
\mathbf{n = 2 1 3}\end{array}$ & $\begin{array}{l}\text { Gesamt } \\
\mathbf{n = 7 0 0}\end{array}$ & $\begin{array}{l}\mathbf{p} \text {-Wert } \\
\text { (Globaltest) }\end{array}$ & $\begin{array}{l}\mathbf{p} \text {-Wert } \\
\text { (Paarvergleiche) }\end{array}$ \\
\hline Ablation & $40(18,8 \%)$ & $32(11,7 \%)$ & $47(22,1 \%)$ & $119(17,0 \%)$ & $\mathbf{0 , 0 0 7}$ & $\begin{array}{l}\text { A-B: } \mathbf{0 , 0 5 8} \\
\text { B-C, } \mathbf{0 0 0}\end{array}$ \\
\hline
\end{tabular}

Tabelle 34: Perioperativ abladierte Patienten (nach Matching) 


\subsubsection{Perioperative Schrittmacherimplantationen}

Bei Betrachtung der Anzahl perioperativer Schrittmacherimplantationen war auch nach Matching ein signifikanter Unterschied nachweisbar. In Gruppe C waren Schrittmacherimplantationen deutlich häufiger notwendig als in Gruppe B und $A$.

\begin{tabular}{|l|l|l|l|l|l|l|}
\hline & $\begin{array}{l}\text { Gruppe A } \\
\mathbf{n = 4 0}\end{array}$ & $\begin{array}{l}\text { Gruppe B } \\
\mathbf{n = 3 2}\end{array}$ & $\begin{array}{l}\text { Gruppe C } \\
\mathbf{n = 4 7}\end{array}$ & $\begin{array}{l}\text { Gesamt } \\
\mathbf{n = 1 1 9}\end{array}$ & $\begin{array}{l}\mathbf{p} \text {-Wert } \\
\text { (Globaltest) }\end{array}$ & $\begin{array}{l}\mathbf{p} \text {-Wert } \\
\text { (Paarvergleiche) }\end{array}$ \\
\hline $\begin{array}{l}\text { Perioperative } \\
\text { SM-Implantation }\end{array}$ & $1(0,5 \%)$ & $8(2,9 \%)$ & $14(6,6 \%)$ & $23(3,3 \%)$ & $\mathbf{0 , 0 0 2 0}$ & $\begin{array}{l}\text { A-B: } \mathbf{0 , 0 9 2} \\
\text { A-C: } \mathbf{0 , 0 0 3} \\
\text { B-C: } \mathbf{0 , 0 9 2}\end{array}$ \\
\hline
\end{tabular}

Tabelle 35: Anzahl perioperativ implantierter Schrittmacher (nach Matching)

Unter den chirurgisch abladierten Patienten (siehe Tabelle 36) benötigten $10,9 \%$ einen Schrittmacher. Dabei war der weit größte Anteil mit 25,5\% in Gruppe C zugegen. Gegenüber Gruppe A mit $0 \%$ und Gruppe B mit 3,1 \% war dies ein signifikantes Ergebnis.

\begin{tabular}{|l|l|l|l|l|l|l|}
\hline & $\begin{array}{l}\text { Gruppe A } \\
\mathbf{n = 4 0}\end{array}$ & $\begin{array}{l}\text { Gruppe B } \\
\mathbf{n = 3 2}\end{array}$ & $\begin{array}{l}\text { Gruppe C } \\
\mathbf{n = 4 7}\end{array}$ & $\begin{array}{l}\text { Gesamt } \\
\mathbf{n = 1 1 9}\end{array}$ & $\begin{array}{l}\mathbf{p} \text {-Wert } \\
\text { (Globaltest) }\end{array}$ & $\begin{array}{l}\text { p-Wert } \\
\text { (Paarvergleiche) }\end{array}$ \\
\hline $\begin{array}{l}\text { Perioperativer } \\
\text { Schrittmacher } \\
\text { nach Ablation }\end{array}$ & $0(0,0 \%)$ & $1(3,1 \%)$ & $12(25,5 \%)$ & $13(10,9 \%)$ & $\mathbf{0 , 0 0 0 1}$ & $\begin{array}{l}\text { A-B: } \mathbf{0 , 2 6 0} \\
\text { A-C: } \mathbf{0 , 0 0 3} \\
\text { B-C: } \mathbf{0 , 0 1 6}\end{array}$ \\
\hline
\end{tabular}

Tabelle 36: Anzahl perioperativ implantierter Schrittmacher nach Ablation (nach Matching)

Unter den nicht abladierten Patienten konnte nach Matching kein signifikanter Unterscheid mehr nachgewiesen werden.

\begin{tabular}{|l|l|l|l|l|l|}
\hline & $\begin{array}{l}\text { Gruppe A } \\
\mathbf{n = 1 7 3}\end{array}$ & $\begin{array}{l}\text { Gruppe B } \\
\mathbf{n = 2 4 2}\end{array}$ & $\begin{array}{l}\text { Gruppe C } \\
\mathbf{n = 1 6 6}\end{array}$ & $\begin{array}{l}\text { Gesamt } \\
\mathbf{n = 5 8 1}\end{array}$ & $\begin{array}{l}\mathbf{p} \text {-Wert } \\
\text { (Globaltest) }\end{array}$ \\
\hline $\begin{array}{l}\text { Perioperative SM-Implantation } \\
\text { ohne Ablation }\end{array}$ & $1(0,6 \%)$ & $7(2,9 \%)$ & $2(1,2 \%)$ & $10(1,7 \%)$ & $\mathbf{0 , 1 6 9}$ \\
\hline
\end{tabular}

Tabelle 37: Anzahl perioperativ implantierter Schrittmacher der nicht abladierten Patienten (nach Matching) 


\subsubsection{Frühsterblichkeit}

Die 30-Tage-Sterblichkeit lag insgesamt bei 2,0 \%.

Intrahospital, aber außerhalb der 30-Tage-Grenze, verstarben 0,6 \% der Patienten.

Nach Matching gab es die Frühsterblichkeit betreffend keinen signifikanten Unterschied mehr zwischen den Gruppen.

\begin{tabular}{|l|l|l|l|l|l|}
\hline & $\begin{array}{l}\text { Gruppe A } \\
\mathbf{n = 2 1 3}\end{array}$ & $\begin{array}{l}\text { Gruppe B } \\
\mathbf{n = 2 7 4}\end{array}$ & $\begin{array}{l}\text { Gruppe C } \\
\mathbf{n = 2 1 3}\end{array}$ & $\begin{array}{l}\text { Gesamt } \\
\mathbf{n = 7 0 0}\end{array}$ & $\mathbf{p}$-Wert (Globaltest) \\
\hline Frühsterblichkeit & & & & & $\mathbf{0 , 3 2 7}$ \\
\hline 30 Tage & $6(2,8 \%)$ & $3(1,1 \%)$ & $5(2,3 \%)$ & $14(2,0 \%)$ & \\
\hline intrahospital > 30 Tage & $2(0,9 \%)$ & $0(0,0 \%)$ & $2(0,9 \%)$ & $4(0,6 \%)$ & \\
\hline
\end{tabular}

Tabelle 38: Frühsterblichkeit (nach Matching)

\subsection{Postoperative Daten (zum Zeitpunkt der Entlassung)}

In diesem Abschnitt werden die postoperativ, d. h. die vor der Entlassung erhobenen Daten der gematchten Studienpopulation betrachtet.

10.4.1 Postoperativer Herzrhythmus

In den nachfolgenden Tabellen 39 bis 42 wird der postoperativ erhobene Herzrhythmus vor der Entlassung aus dem stationären klinischen Aufenthalt dargestellt. Auch hier sind Patienten mit Herzschrittmacher inkludiert. Beschrieben wird der Herzrhythmus aller Patienten zusammengefasst sowie der Abladierten und jener mit präoperativem Sinusrhythmus. Insgesamt hatten bei Entlassung 16,6 \% der Patienten Vorhofflimmern, gegenüber den 23,7 \% vor dem Matching. Der Unterschied zwischen den Gruppen zeigte sich nicht mehr signifikant. 


\begin{tabular}{|l|l|l|l|l|l|}
\hline & $\begin{array}{l}\text { Gruppe A } \\
\mathbf{n = 2 0 3}\end{array}$ & $\begin{array}{l}\text { Gruppe B } \\
\mathbf{n = 2 7 2}\end{array}$ & $\begin{array}{l}\text { Gruppe C } \\
\mathbf{n = 2 0 7}\end{array}$ & $\begin{array}{l}\text { Gesamt } \\
\mathbf{n = 6 8 2}\end{array}$ & $\mathbf{p}$-Wert (Globaltest) \\
\hline Postoperativer Herzrhythmus & & & & & $\mathbf{0 , 2 4 1}$ \\
\hline SR & $176(86,7 \%)$ & $220(80,9 \%)$ & $173(83,6 \%)$ & $569(83,4 \%)$ & \\
\hline VHF & $27(13,3 \%)$ & $52(19,1 \%)$ & $34(16,4 \%)$ & $113(16,6 \%)$ & \\
\hline
\end{tabular}

Tabelle 39: Postoperativer Herzrhythmus bei Entlassung (nach Matching)

Von den Patienten, die aufgrund von präoperativem Vorhofflimmern chirurgisch abladiert wurden, hatten nach Matching 28,6 \% gegenüber vorherigen 36,7 \% aller Patienten bei Entlassung Vorhofflimmern.

Auch nach Matching war der Ablationserfolg in Gruppe B am geringsten und in Gruppe A am größten. Dieser Unterschied blieb weiterhin signifikant.

\begin{tabular}{|l|l|l|l|l|l|l|}
\hline & $\begin{array}{l}\text { Gruppe A } \\
\mathbf{n}=35\end{array}$ & $\begin{array}{l}\text { Gruppe B } \\
\mathbf{n = 3 2}\end{array}$ & $\begin{array}{l}\text { Gruppe C } \\
\mathbf{n}=\mathbf{4 5}\end{array}$ & $\begin{array}{l}\text { Gesamt } \\
\mathbf{n = 1 1 2}\end{array}$ & $\begin{array}{l}\text { p-Wert } \\
\text { (Globaltest) }\end{array}$ & $\begin{array}{l}\text { p-Wert } \\
\text { (Paarvergleiche) }\end{array}$ \\
\hline $\begin{array}{l}\text { Postoperativer } \\
\text { Rhythmus } \\
\text { nach Ablation }\end{array}$ & & & & $\mathbf{0 , 0 1 2}$ & $\begin{array}{l}\text { A-B: } \mathbf{0 , 0 0 9} \\
\text { A-C: } \mathbf{0 , 0 7 2} \\
\text { B-C: } \mathbf{0 , 2 5 6}\end{array}$ \\
\hline SR & $31(88,6 \%)$ & $18(56,3 \%)$ & $31(68,9 \%)$ & $80(71,4 \%)$ & & \\
\hline VHF & $4(11,4 \%)$ & $14(43,8 \%)$ & $14(31,1 \%)$ & $32(28,6 \%)$ & & \\
\hline
\end{tabular}

Tabelle 40: Postoperativer Herzrhythmus nach Ablation bei Entlassung (nach Matching)

Ohne Ablation gab es nach Matching keinen signifikanten Unterschied zwischen den Gruppen.

\begin{tabular}{|l|l|l|l|l|l|}
\hline & $\begin{array}{l}\text { Gruppe A } \\
n=168\end{array}$ & $\begin{array}{l}\text { Gruppe B } \\
n=240\end{array}$ & $\begin{array}{l}\text { Gruppe C } \\
n=162\end{array}$ & $\begin{array}{l}\text { Gesamt } \\
\mathbf{n = 5 7 0}\end{array}$ & $\begin{array}{l}\text { p-Wert } \\
\text { (Globaltest) }\end{array}$ \\
\hline $\begin{array}{l}\text { Postoperativer Rhythmus } \\
\text { ohne Ablation }\end{array}$ & & & & & $\mathbf{0 , 6 0 1}$ \\
\hline SR & $145(86,3 \%)$ & $202(84,2 \%)$ & $142(87,7 \%)$ & $489(85,8 \%)$ & \\
\hline VHF & $23(13,7 \%)$ & $38(15,8 \%)$ & $20(12,3 \%)$ & $81(14,2 \%)$ & \\
\hline
\end{tabular}

Tabelle 41: Postoperativer Herzrhythmus der nicht Abladierten bei Entlassung (nach Matching) 
Postoperativ neu aufgetretenes Vorhofflimmern unterschied sich auch nach Matching weiterhin nicht signifikant in den Gruppen.

\begin{tabular}{|l|l|l|l|l|l|}
\hline & $\begin{array}{l}\text { Gruppe A } \\
\mathbf{n = 1 3 7}\end{array}$ & $\begin{array}{l}\text { Gruppe B } \\
\mathbf{n = 2 0 6}\end{array}$ & $\begin{array}{l}\text { Gruppe C } \\
\mathbf{n = 1 4 5}\end{array}$ & $\begin{array}{l}\text { Gesamt } \\
\mathbf{n = 4 8 8}\end{array}$ & $\begin{array}{l}\text {-Wert } \\
\text { (Globaltest) }\end{array}$ \\
\hline $\begin{array}{l}\text { Postoperativer Rhythmus bei } \\
\text { präoperativem SR }\end{array}$ & & & $\mathbf{0 , 3 6 9}$ \\
\hline SR & $126(92,0 \%)$ & $180(87,4 \%)$ & $131(90,3 \%)$ & $437(89,5 \%)$ & \\
\hline Vorhofflimmern & $11(8,0 \%)$ & $26(12,6 \%)$ & $14(9,7 \%)$ & $51(10,5 \%)$ & \\
\hline
\end{tabular}

Tabelle 42: Postoperativer Herzrhythmus der Patienten mit präoperativem Sinusrhythmus (nach Matching)

\subsubsection{Postoperative Schrittmacheranzahl}

Eingeschlossen wurden hier alle Patienten, die präoperativ schon mit einem Schrittmacher versorgt worden waren oder perioperativ einen bekommen hatten.

Wenngleich hier nach Matching kein signifikanter Unterschied mehr vorhanden war, war der prozentuale Anteil an Vorhofflimmern in Gruppe $\mathrm{C}$ und B deutlich höher als in Gruppe A. Diese Zahlen kamen durch den in Gruppe B erhöhten Anteil präoperativer Schrittmacherpatienten und die in Gruppe C höhere Zahl der perioperativen Implantationen zustande.

\begin{tabular}{|l|l|l|l|l|l|}
\hline & $\begin{array}{l}\text { Gruppe A } \\
\mathbf{n = 2 1 3}\end{array}$ & $\begin{array}{l}\text { Gruppe B } \\
\mathbf{n = 2 7 4}\end{array}$ & $\begin{array}{l}\text { Gruppe C } \\
\mathbf{n = 2 1 3}\end{array}$ & $\begin{array}{l}\text { Gesamt } \\
\mathbf{n = 7 0 0}\end{array}$ & $\begin{array}{l}\mathbf{p} \text {-Wert } \\
\text { (Globaltest) }\end{array}$ \\
\hline $\begin{array}{l}\text { Schrittmacher postoperativ } \\
\text { gesamt }\end{array}$ & $8(3,8 \%)$ & $23(8,4 \%)$ & $19(8,8 \%)$ & $51(7,3 \%)$ & $\mathbf{0 , 0 5 4}$ \\
\hline
\end{tabular}

Tabelle 43: Schrittmacheranzahl bei Entlassung (nach Matching) 


\subsection{Follow-up}

10.5.1 Teilnahme und mittlere Follow-up-Zeit

Insgesamt konnte bei 633 der 682 überlebenden Patienten (92,8 \%) ein Followup erhoben werden. Bei den fehlenden 91 Patienten war es trotz intensiver Bemühungen nicht möglich, ein Follow-up zu generieren.

\begin{tabular}{|l|l|l|l|l|}
\hline & $\begin{array}{l}\text { Gruppe A } \\
\mathbf{n = 2 0 5}\end{array}$ & $\begin{array}{l}\text { Gruppe B } \\
\mathbf{n = 2 7 1}\end{array}$ & $\begin{array}{l}\text { Gruppe C } \\
\mathbf{n = 2 0 6}\end{array}$ & $\begin{array}{l}\text { Gesamt } \\
\mathbf{n = 6 8 2}\end{array}$ \\
\hline Follow-up-Anzahl & $185(90,2 \%)$ & $252(93,0 \%)$ & $196(95,1 \%)$ & $633(92,8 \%)$ \\
\hline
\end{tabular}

Tabelle 44: Follow-up-Anzahl unter den Überlebenden (nach Matching)

Die durchschnittliche Follow-up-Zeit (von der Entlassung bis zum letzten Follow-up) betrug 83,8 $\pm 50,4$ Monate.

\begin{tabular}{|c|c|c|c|c|c|c|}
\hline & $\begin{array}{l}\text { Gruppe A } \\
n=185\end{array}$ & $\begin{array}{l}\text { Gruppe B } \\
n=252\end{array}$ & $\begin{array}{l}\text { Gruppe C } \\
n=196\end{array}$ & $\begin{array}{l}\text { Gesamt } \\
n=633\end{array}$ & $\begin{array}{l}\text { p-Wert } \\
\text { (Globaltest) }\end{array}$ & $\begin{array}{l}\text { p-Wert } \\
\text { (Paarvergleiche }\end{array}$ \\
\hline FU-Zeit & & & & & $<0,0001$ & $\begin{array}{l}\text { A-B: }<0,001 \\
\text { A-C: }<0,001 \\
\text { B-C: }<0,001\end{array}$ \\
\hline Mittelwert \pm SD & $130,5 \pm 46,2$ & $47,1 \pm 31,8$ & $86,8 \pm 32,9$ & $83,8 \pm 50,4$ & & \\
\hline Minimum & 11 & 0 & 2 & 0 & & \\
\hline Maximum & 217 & 146 & 151 & 217 & & \\
\hline
\end{tabular}

Tabelle 45: Mittlere Follow-up-Zeit (nach Matching)

10.5.2 Kumulative Follow-up-Zeit

Die gesamte Beobachtungszeit von Entlassung bis zum letzten Follow-up betrug 4419 Patientenjahre. In Gruppe A waren es 2012 Patientenjahre, in Gruppe B 989 Patientenjahre und in Gruppe C 1417 Patientenjahre. 


\subsubsection{Langzeitbeobachtungen}

Um Aussagen über den Langzeitherzrhythmus oder die Notwendigkeit von Schrittmacherimplantationen in den Jahren nach der Operation machen zu können, wurden die erhobenen Daten des letzten Follow-ups eines jeden der 633 Patienten ausgewertet.

10.5.4 Herzrhythmus im letzten Follow-up (inkl. Grundrhythmus bei Schrittmacher)

Auch hier wurde der Grundrhythmus des Herzens betrachtet; das bedeutet: Auch Patienten mit Herzschrittmacher sind inkludiert. In der Langzeitbeobachtung nach Matching gab es keinen signifikanten Unterschied in der Häufigkeit von Vorhofflimmern mehr.

\begin{tabular}{|l|l|l|l|l|l|}
\hline & $\begin{array}{l}\text { Gruppe A } \\
\mathbf{n = 1 8 5}\end{array}$ & $\begin{array}{l}\text { Gruppe B } \\
\mathbf{n = 2 5 2}\end{array}$ & $\begin{array}{l}\text { Gruppe C } \\
\mathbf{n = 1 9 6}\end{array}$ & $\begin{array}{l}\text { Gesamt } \\
\mathbf{n = 6 3 3}\end{array}$ & $\begin{array}{l}\text { p-Wert } \\
\text { (Globaltest) }\end{array}$ \\
\hline Rhythmus im letzten FU & & & $\mathbf{0 , 1 5 1}$ \\
\hline SR & $122(65,9 \%)$ & $183(72,6 \%)$ & $146(74,5 \%)$ & $451(71,2 \%)$ & \\
\hline VHF & $63(34,1 \%)$ & $69(27,4 \%)$ & $50(25,5 \%)$ & $182(28,8 \%)$ & \\
\hline
\end{tabular}

Tabelle 46:Herzrhythmus im letzten Follow-up (nach Matching)

In Tabelle 47 wurden von den 119 abladierten Patienten nur die 102 betrachtet, von denen ein Follow-up vorlag. Die Langzeitergebnisse nach Ablation zeigten nach Matching keinen signifikanten Unterschied mehr.

\begin{tabular}{|l|l|l|l|l|l|}
\hline & $\begin{array}{l}\text { Gruppe A } \\
\mathbf{n = 3 5}\end{array}$ & $\begin{array}{l}\text { Gruppe B } \\
\mathbf{n = 2 9}\end{array}$ & $\begin{array}{l}\text { Gruppe C } \\
\mathbf{n = 3 8}\end{array}$ & $\begin{array}{l}\text { Gesamt } \\
\mathbf{n = 1 0 2}\end{array}$ & $\begin{array}{l}\mathbf{p} \text {-Wert } \\
\text { (Globaltest) }\end{array}$ \\
\hline $\begin{array}{l}\text { Rhythmus im letzten FU } \\
\text { nach Ablation }\end{array}$ & & & & & $\mathbf{0 , 1 7 2}$ \\
\hline SR & $13(37,1 \%)$ & $12(41,4 \%)$ & $22(57,9 \%)$ & $47(46,1 \%)$ & \\
\hline VHF & $22(62,9 \%)$ & $17(58,6 \%)$ & $16(42,1 \%)$ & $55(53,9 \%)$ & \\
\hline
\end{tabular}

Tabelle 47: Herzrhythmus im letzten Follow-up nach Ablation (nach Matching) 
Hieraus errechnet sich eine Inzidenz für ein Vorhofflimmerrezidiv nach Ablation von 1,1 \% für Gruppe A, 1,6 \% für Gruppe B und 1,1 \% für Gruppe C pro Patientenjahr.

Für die Berechnung wurde die kumulative Follow-up-Zeit der Gruppen verwendet.

Bei den verbleibenden nicht abladierten 531 Patienten gab es ebenfalls keinen signifikanten Unterschied zwischen den Gruppen.

\begin{tabular}{|l|l|l|l|l|l|}
\hline & $\begin{array}{l}\text { Gruppe A } \\
\mathbf{n = 1 5 0}\end{array}$ & $\begin{array}{l}\text { Gruppe B } \\
\mathbf{n = 2 2 3}\end{array}$ & $\begin{array}{l}\text { Gruppe C } \\
\mathbf{n = 1 5 8}\end{array}$ & $\begin{array}{l}\text { Gesamt } \\
\mathbf{n = 5 3 1}\end{array}$ & $\begin{array}{l}\text {-Wert } \\
\text { (Globaltest) }\end{array}$ \\
\hline $\begin{array}{l}\text { Rhythmus im letzten FU } \\
\text { ohne Ablation }\end{array}$ & $109(72,7 \%)$ & $171(76,7 \%)$ & $124(78,5 \%)$ & $404(76,1 \%)$ & $\mathbf{0 , 4 7 1 0}$ \\
\hline SR & $41(27,3 \%)$ & $52(23,3 \%)$ & $34(21,5 \%)$ & $127(23,9 \%)$ & \\
\hline VHF & & & & \\
\hline
\end{tabular}

Tabelle 48: Herzrhythmus im letzten Follow-up der nicht Abladierten (nach Matching)

Tabelle 49 zeigt die Patienten, die vor der Operation nicht an Herzrhythmusstörungen gelitten hatten, also einen Sinusrhythmus hatten. Daraus ließ sich der Anteil an nach der Operation neu aufgetretenem Vorhofflimmern bestimmen. Dieser zeigte im letzten Follow-up auch nach Matching keinen signifikanten Unterschied zwischen den Gruppen.

\begin{tabular}{|l|l|l|l|l|l|}
\hline & $\begin{array}{l}\text { Gruppe A } \\
\mathbf{n = 1 2 4}\end{array}$ & $\begin{array}{l}\text { Gruppe B } \\
\mathbf{n = 1 9 3}\end{array}$ & $\begin{array}{l}\text { Gruppe C } \\
\mathbf{n = 1 4 2}\end{array}$ & $\begin{array}{l}\text { Gesamt } \\
\mathbf{n = 4 5 9}\end{array}$ & $\begin{array}{l}\text { p-Wert } \\
\text { (Globaltest) }\end{array}$ \\
\hline $\begin{array}{l}\text { Rhythmus im letzten FU } \\
\text { bei präoperativem SR }\end{array}$ & & & & 0,967 \\
\hline SR & $101(81,5 \%)$ & $157(81,3 \%)$ & $117(82,4 \%)$ & $375(81,7 \%)$ & \\
\hline Vorhofflimmern & $23(18,5 \%)$ & $36(18,7 \%)$ & $25(17,6 \%)$ & $84(18,3 \%)$ & \\
\hline
\end{tabular}

Tabelle 49: Herzrhythmus der Patienten mit präoperativem Sinusrhythmus zum Zeitpunkt des letzten Follow-ups (nach Matching) 
Hieraus errechnet sich eine Inzidenz für neu aufgetretenes Vorhofflimmern von 1,1 \% für Gruppe A, 3,6 \% für Gruppe B und 1,7 \% für Gruppe C pro Patientenjahr.

Die Berechnung erfolgte anhand der kumulativen Follow-up-Zeit von 2012 Patientenjahren in Gruppe A, 989 in Gruppe B und 1417 in Gruppe C.

10.5.4.1 Anteil an Vorhofflimmern im Beobachtungszeitraum

Nun folgt in den Abbildungen 12 und 13 eine grafische Übersicht des Vorhofflimmeranteils im Verlauf.

In Abbildung 12 wurden alle 700 Patienten betrachtet, inklusive der abladierten und der nicht abladierten. Präoperativ war bei der gematchten Population der Anteil an Vorhofflimmern in Gruppe A am größten. Postoperativ konnte bei allen drei Gruppen ein deutlicher Rückgang der Vorhofflimmerrate beobachtet werden.

Zum Zeitpunkt des letzten Follow-ups zeigte sich eine hohe Rezidivrate an Vorhofflimmern.

Im Gegensatz zur Auswertung der ungematchten Population gab es nach Matching zu allen drei Zeitpunkten keinen signifikanten Unterschied mehr zwischen den Gruppen. 


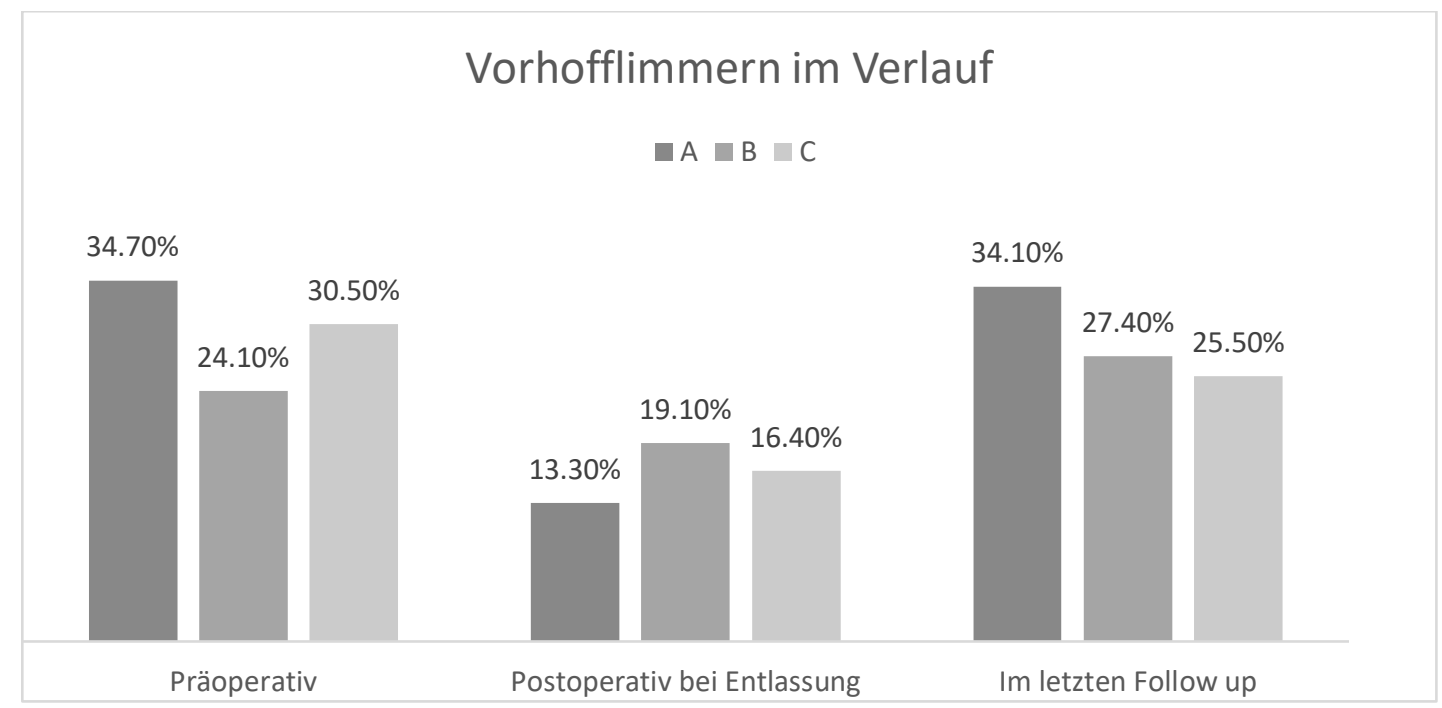

Abbildung 12: Vorhoffimmeranteil der Vergeichsgruppe (nach Matching) im Verlauf

In Abbildung 13 wurden nur die abladierten Patienten im Hinblick auf den primären Ablationserfolg bei Entlassung und den Langzeiterfolg im letzten Follow-up betrachtet. Als Ablationserfolg wurde hierbei die Abwesenheit von Vorhofflimmern, also ein wiederhergestellter Sinusrhythmus gewertet.

Auch nach Matching war der primäre Ablationserfolg in Gruppe A signifikant am größten $(88,6 \%)$ und in Gruppe B am kleinsten (56,2\%).

Weiterhin blieb auch die Rezidivrate mit 51,5\% in Gruppe A am größten, wobei wiederum die längste Follow-up-Zeit in dieser Gruppe berücksichtigt werden muss.

In den absoluten Anteilen an Vorhofflimmern unter den Patienten im letzten Follow-up gab es unter den Gruppen keinen signifikanten Unterschied. 


\section{Vorhofflimmern nach Ablation}

$\square=\mathrm{B} \square \mathrm{C}$
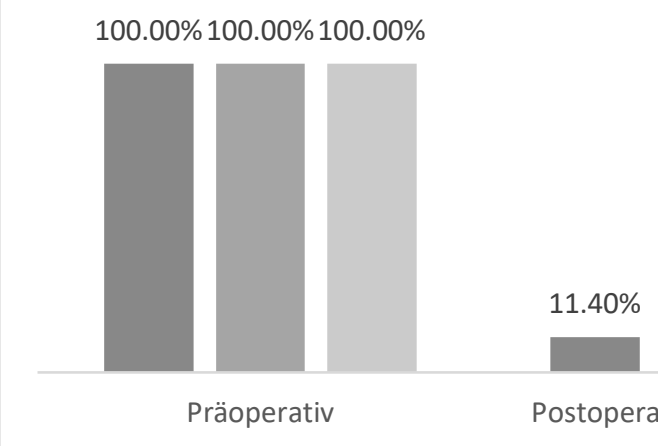

$43.80 \%$

$11.40 \%$

Postoperativ bei Entlassung

$62.90 \% \quad 58.60 \%$

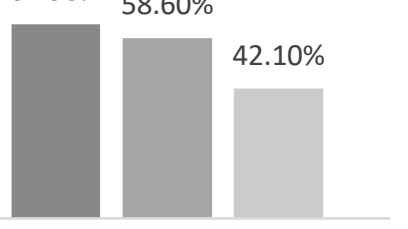

Im letzten Follow up

Abbildung 13: Vorhofflimmeranteil der Vergleichsgruppen nach Ablation (nach Matching) im Verlauf

\subsubsection{Schrittmacher im letzten Follow-up}

Die Schrittmacheranzahlen im letzten Follow-up nach Matching wiesen keinen signifikanten Unterschied zwischen den Gruppen auf.

\begin{tabular}{|l|l|l|l|l|l|l|}
\hline & $\begin{array}{l}\text { Gruppe A } \\
\mathbf{n = 1 8 5}\end{array}$ & $\begin{array}{l}\text { Gruppe B } \\
\mathbf{n = 2 5 2}\end{array}$ & $\begin{array}{l}\text { Gruppe C } \\
\mathbf{n = 1 9 6}\end{array}$ & $\begin{array}{l}\text { Gesamt } \\
\mathbf{n = 6 3 3}\end{array}$ & $\begin{array}{l}\mathbf{p} \text {-Wert } \\
\text { (Globaltest) }\end{array}$ & $\begin{array}{l}\mathbf{p} \text {-Wert } \\
\text { (Paarvergleiche) }\end{array}$ \\
\hline $\begin{array}{l}\text { Schrittmacher im } \\
\text { letzten FU }\end{array}$ & $\begin{array}{l}24 \\
(13,0 \%)\end{array}$ & $\begin{array}{l}36 \\
(14,3 \%)\end{array}$ & $\begin{array}{l}38 \\
(19,4 \%)\end{array}$ & $\begin{array}{l}98 \\
(15,4 \%)\end{array}$ & $\mathbf{0 , 1 7 8}$ & \\
\hline $\begin{array}{l}\text { Patienten mit nach } \\
\text { Entlassung indiziertem } \\
\text { Schrittmacher im } \\
\text { letzten FU }\end{array}$ & $\begin{array}{l}18 \\
(9,7 \%)\end{array}$ & $\begin{array}{l}22 \\
(8,7 \%)\end{array}$ & $\begin{array}{l}33 \\
(16,8 \%)\end{array}$ & $\begin{array}{l}73 \\
(11,5 \%)\end{array}$ & $\mathbf{0 , 0 2 5}$ & $\begin{array}{l}\text { A-C: } \mathbf{0 , 0 8 8} \\
\text { B-C: } \mathbf{0 , 0 3 9}\end{array}$ \\
\hline
\end{tabular}

Tabelle 50: Absolute Schrittmacherzahl zum Zeitpunkt des letzten Follow-ups (nach Matching)

Hieraus errechnet sich eine Inzidenz für Schrittmacherimplantationen von 0,8 \% für Gruppe A, 2,2 \% für Gruppe B und 2,3 \% für Gruppe C pro Patientenjahr. Die Berechnung erfolgte anhand der kumulativen Follow-up-Zeit von 2012 Patientenjahren in Gruppe A, 989 in Gruppe B und 1417 in Gruppe C. 
10.5.5.1 Anteil an Schrittmachern im Beobachtungszeitraum

In der folgenden Abbildung wird eine Übersicht der Schrittmacheranzahlen zu verschiedenen Zeitpunkten des Beobachtungszeitraums gegeben. Daraus konnte auf den Anteil der Neuimplantationen im jeweiligen Zeitabschnitt rückgeschlossen werden.

Präoperativ gab es keinen signifikanten Unterschied zwischen den Gruppen, wenngleich in Gruppe B die meisten Patienten mit einem schon vorhandenen Schrittmacher waren.

Die postoperative Spalte stellt die Gesamtzahl der Patienten mit Schrittmacher zum Zeitpunkt der Entlassung dar, das bedeutet: präoperativ vorhandene zuzüglich perioperativ implantierter Schrittmacher.

Daraus lässt sich erkennen, dass die meisten perioperativen Schrittmacherimplantationen (wie auch schon vor dem Matching) in Gruppe $C$ vorkamen (6,6 \%), gefolgt von Gruppe B (2,9\%). Bei Gruppe A wurde einem Patienten perioperativ ein Schrittmacher implantiert; der Patient verstarb jedoch perioperativ, so dass bei Entlassung der prozentuale Anteil idem zum präoperativen Status war.

Gruppe A hatte somit, wie schon beschrieben, signifikant weniger perioperative Schrittmacherimplantationen als die anderen beiden Gruppen.

Im Gegensatz zu den Ergebnissen der ungematchten Population waren die postoperativen und im letzten Follow-up gezeigten Unterschiede zwischen den Gruppen nach Matching nicht mehr signifikant.

Die Differenz aus Schrittmachern bei Entlassung und der Anzahl derselben im letzten Follow-up ergibt die Zahl der Neuimplantationen im Beobachtungszeitraum.

Diese betrug bei Gruppe C 10,6 \%, gefolgt von Gruppe A mit 9,2\%. Bei Gruppe B waren es 5,9\% Schrittmacherimplantationen zwischen Entlassung und letztem Follow-up. 
In der ungematchten Studienpopulation hatten sich die meisten Neuimplantationen nach Entlassung bei Gruppe $C$ gezeigt, in absteigender Reihenfolge gefolgt von Gruppe B, die wenigsten bei Gruppe A. Nach Matching scheint wie auch vorher schon Gruppe $C$ sowohl perioperativ als auch in der Langzeitbeobachtung mit der höchsten Zahl an Schrittmachern den anderen beiden Gruppen unterlegen zu sein.

Wenngleich die Zahl der Neuimplantationen im Beobachtungszeitraum nach Entlassung bei Gruppe A höher ist als vor dem Matching, sind die absoluten Zahlen perioperativ, postoperativ und im letzten Follow up im Vergleich zu den anderen Gruppen doch am geringsten.

In Bezug auf die Notwendigkeit von Schrittmacherimplantationen scheint Gruppe A daher den anderen beiden Gruppen überlegen zu sein.

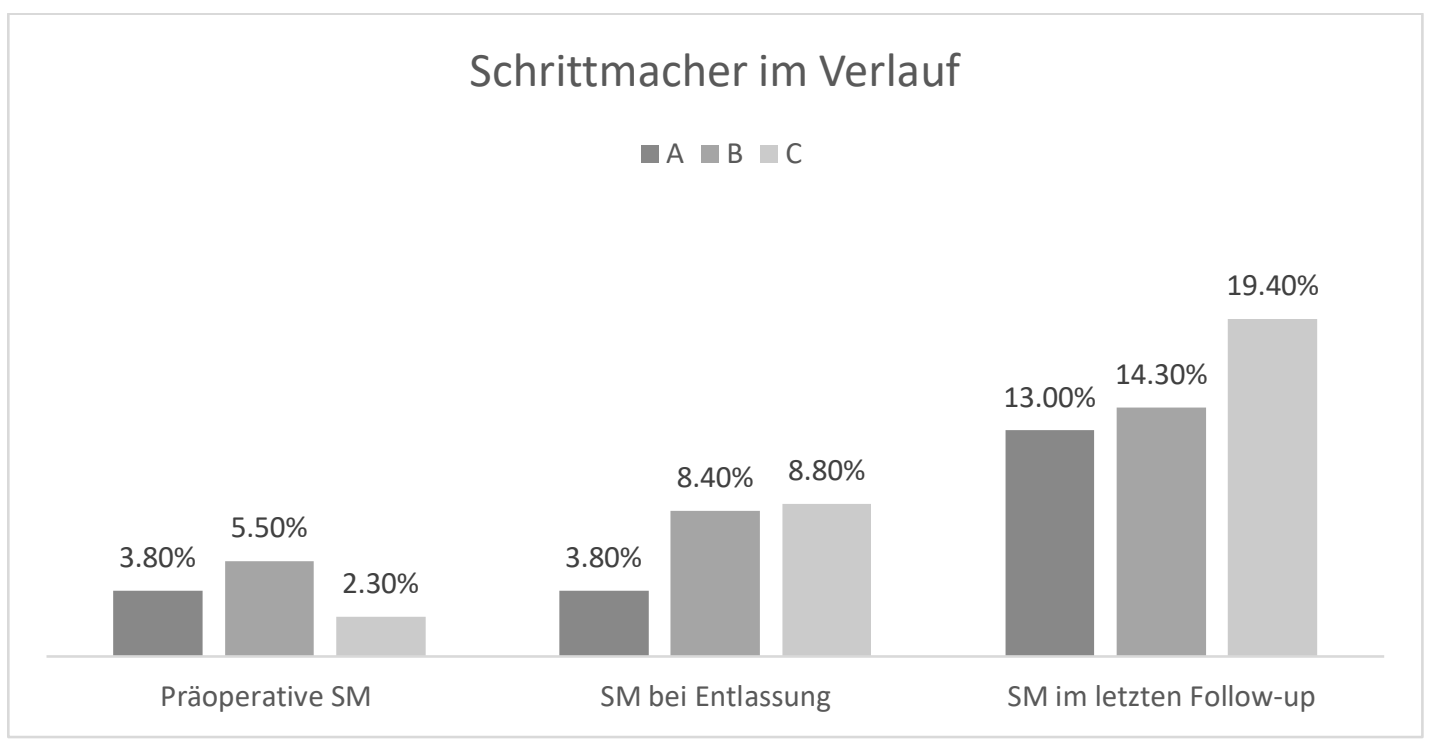

Abbildung 14: Schrittmacheranteil der Vergleichsgruppen (nach Matching) im Verlauf

10.5.5.1.1 Ereignisanzahl Schrittmacher und Defibrillatorimplantationen

Als Ereignis wurde hier ein nach der Operation, also im Zeitraum perioperativ bis zum letzten Follow-up, implantierter Schrittmacher oder Defibrillator 
definiert. Die Zeitspanne reichte dabei jeweils von dem Datum der Operation bis zum Implantationsdatum oder Zeitpunkt des letzten Kontaktes.

Wie auch schon in der ungematchten Studienpopulation gab es bei Gruppe $C$ signifikant mehr Schrittmacherereignisse als in den beiden anderen Gruppen.

\begin{tabular}{|l|l|l|l|l|l|l|}
\hline & $\begin{array}{l}\text { Gruppe A } \\
\mathbf{n = 2 1 3}\end{array}$ & $\begin{array}{l}\text { Gruppe B } \\
\mathbf{n = 2 7 4}\end{array}$ & $\begin{array}{l}\text { Gruppe C } \mathbf{n} \\
\mathbf{2} \mathbf{2 1 3}\end{array}$ & $\begin{array}{l}\text { Gesamt } \\
\mathbf{n = 7 0 0}\end{array}$ & $\begin{array}{l}\text { p-Wert } \\
\text { (Globaltest) }\end{array}$ & $\begin{array}{l}\text { p-Wert } \\
\text { (Paarvergleiche) }\end{array}$ \\
\hline $\begin{array}{l}\text { Schrittmacher- } \\
\text { Implantationen } \\
\text { gesamt }\end{array}$ & $18(8,5 \%)$ & $22(8,0 \%)$ & $34(16,0 \%)$ & $74(10,6 \%)$ & $\mathbf{0 , 0 0 9}$ & $\begin{array}{l}\text { A-B: } \mathbf{0 , 8 6 7} \\
\text { A-C: } \mathbf{0 , 0 3 6}\end{array}$ \\
\hline Nach Ablation & $7(3,3 \%)$ & $3(1,1 \%)$ & $16(7,5 \%)$ & $26(3,7 \%)$ & & \\
\hline $\begin{array}{l}\text { Defibrillator } \\
\text { Implantationen } \\
\text { Gesamt }\end{array}$ & $2(0,9 \%)$ & $4(1,5 \%)$ & $4(1,9 \%)$ & $10(1,4 \%)$ & $\mathbf{0 , 7 1 5}$ & \\
\hline
\end{tabular}

Tabelle 51: Ereignisanzahl Schrittmacher- und Defibrillatorimplantationen (nach Matching)

\subsection{Kaplan-Meier-Schätzung der Schrittmacherhäufigkeit}

Unter den gematchten Patienten waren es insgesamt 74 der 700 Patienten, die während oder nach der Operation mit einem Schrittmacher versorgt werden mussten.

In der Kaplan-Meier-Kurve wird deutlich sichtbar, dass Gruppe A gegenüber Gruppe B und C in Bezug auf die Schrittmacherfreiheit signifikant überlegen war.

Nach 5 Jahren waren bei Gruppe A noch $98 \pm 1 \%$ der Patienten ohne Schrittmacher. In Gruppe B waren es $89 \pm 2 \%$, in Gruppe C $87 \pm 2 \%$.

Nach 10 Jahren waren es in Gruppe A immer noch $92 \pm 2 \%$, in Gruppe B $87 \pm$ $3 \%$ und in Gruppe C $78 \pm 4 \%$.

Sichtbar wird auch, dass die Zahl der perioperativen

Schrittmacherimplantationen in Gruppe $\mathrm{C}$ und B deutlich höher war als in Gruppe A.

Die Kurve zeigt nach Matching keine wesentlich anderen Ergebnisse als vor dem Matching, sondern nur geringfügig andere Zahlen. 


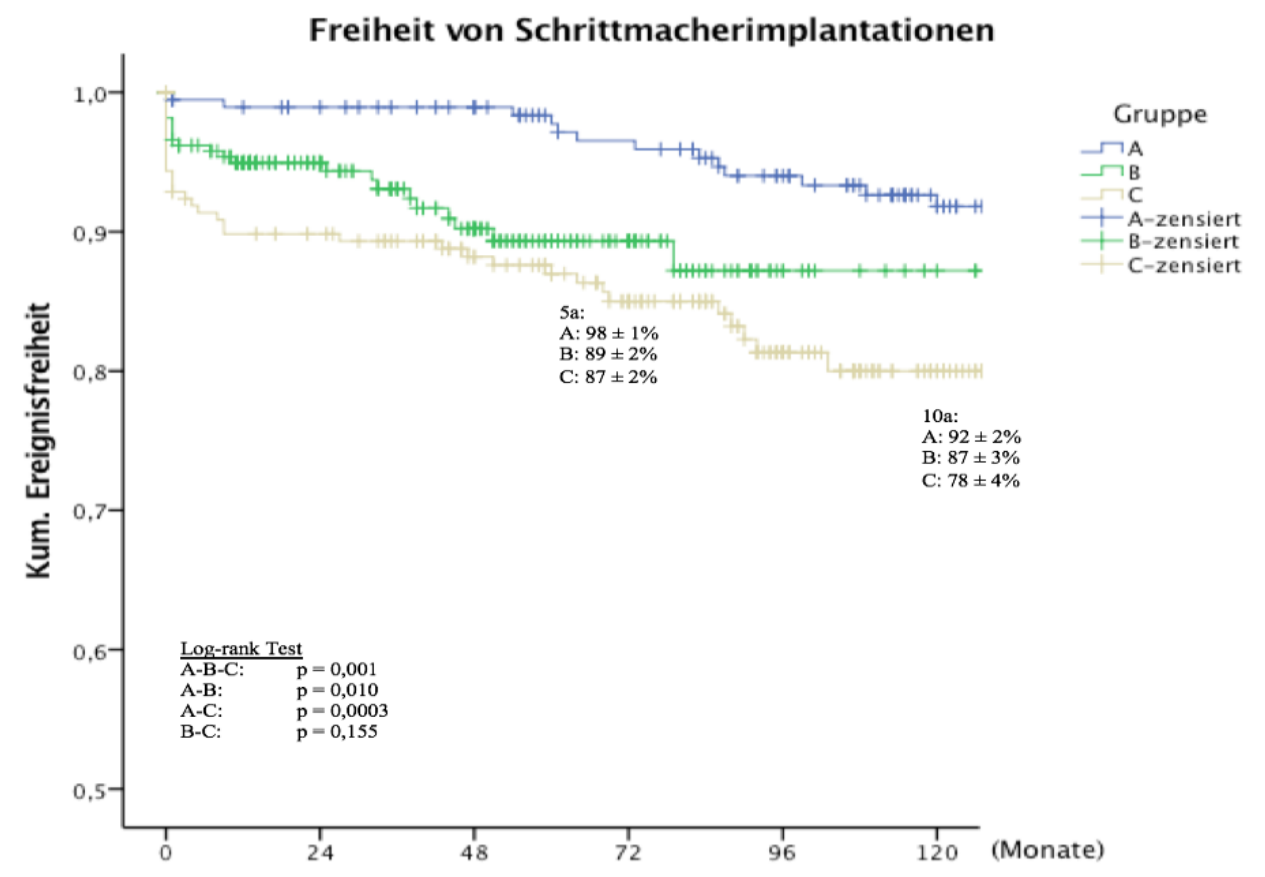

Patienten unter Risiko ( $n$ )

$\begin{array}{lcccccc}\text { Gruppe A } & 199 & 178 & 168 & 152 & 129 & 92 \\ \text { Gruppe B } & 226 & 145 & 90 & 38 & 11 & 4 \\ \text { Gruppe C } & 205 & 165 & 141 & 102 & 54 & 16\end{array}$

Abbildung 15: Kaplan-Meier-Kurve: Freiheit von Schrittmacherimplantationen (nach Matching)

\begin{tabular}{|l|l|l|l|}
\hline Zeit in Monaten & \multicolumn{3}{|l|}{ Kumulierter Anteil der Schrittmacherfreien \pm SE in \% } \\
\hline & Gruppe A & Gruppe B & Gruppe C \\
\hline 24 & $99 \pm 1$ & $94 \pm 2$ & $90 \pm 2$ \\
\hline 48 & $99 \pm 1$ & $90 \pm 2$ & $88 \pm 2$ \\
\hline 72 & $97 \pm 1$ & $89 \pm 2$ & $85 \pm 3$ \\
\hline 96 & $94 \pm 2$ & $86 \pm 3$ & $82 \pm 3$ \\
\hline 120 & $93 \pm 2$ & $86 \pm 3$ & $80 \pm 3$ \\
\hline
\end{tabular}

Tabelle 52: Kumulative Schrittmacherfreiheit (nach Matching) 
10.5.5.1.3 Kaplan-Meier-Schätzung der Defibrillatorhäufigkeit

Bei 10 Patienten wurde im Beobachtungszeitraum ein Defibrillator implantiert. Es zeigte sich kein signifikanter Unterschied zwischen den Gruppen.

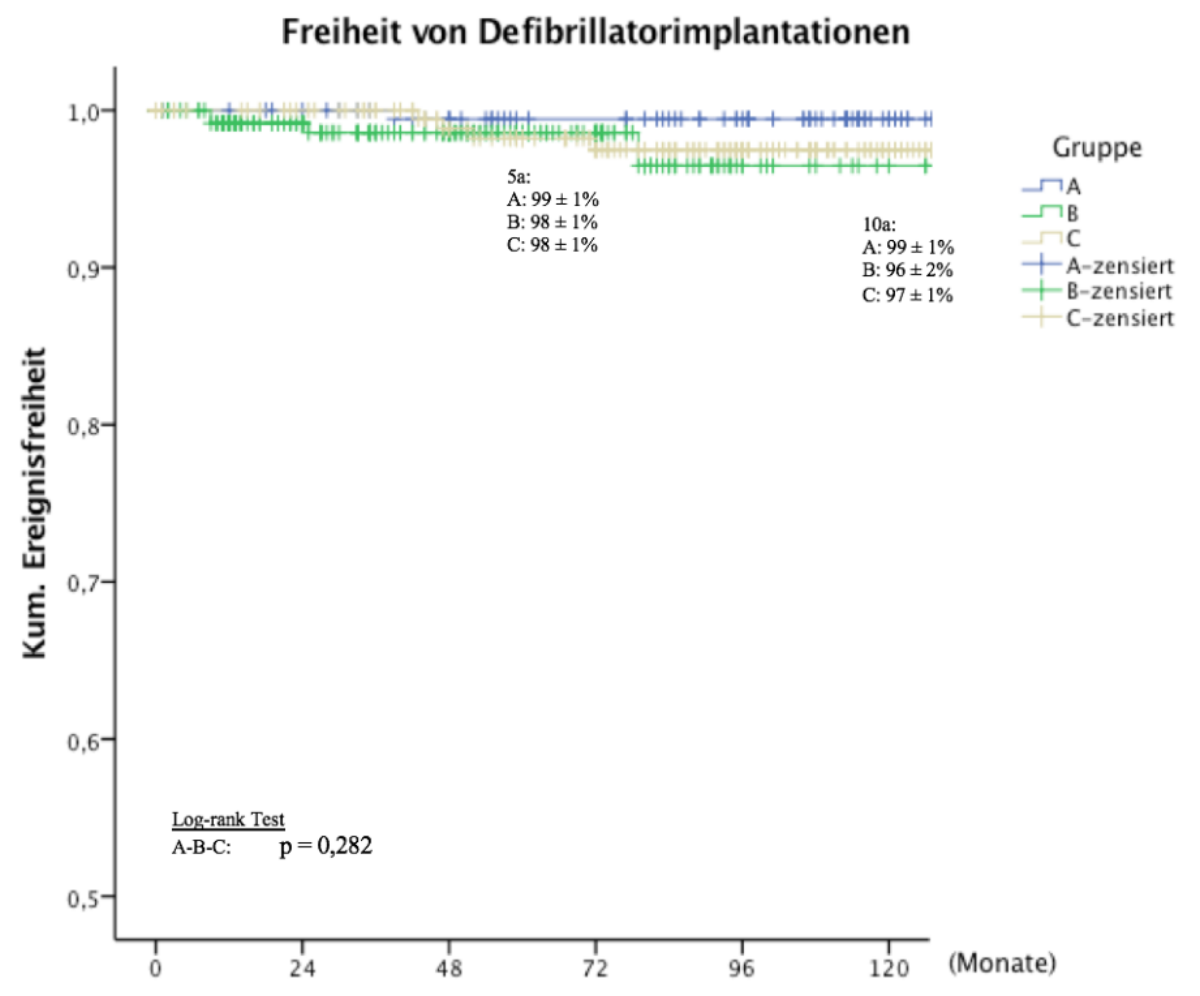

Patienten unter Risiko ( $\mathrm{n}$ )

$\begin{array}{ccccccc}\text { Gruppe A } & 198 & 179 & 168 & 155 & 134 & 97 \\ \text { Gruppe B } & 224 & 149 & 97 & 43 & 12 & 4 \\ \text { Gruppe C } & 201 & 177 & 150 & 111 & 60 & 20\end{array}$

Abbildung 16: Kaplan-Meier-Kurve: Freiheit von Defibrillatorimplantationen (nach Matching) 


\begin{tabular}{|l|l|l|l|}
\hline Zeit in Monaten & \multicolumn{4}{|l|}{ Kumulierter Anteil der Defibrillatorfreien \pm SE in \% } \\
\hline & Gruppe A & Gruppe B & Gruppe C \\
\hline 24 & $100 \pm 0$ & $99 \pm 1$ & $100 \pm 0$ \\
\hline 48 & $99 \pm 1$ & $98 \pm 1$ & $99 \pm 1$ \\
\hline 72 & $99 \pm 1$ & $98 \pm 1$ & $98 \pm 1$ \\
\hline 96 & $99 \pm 1$ & $96 \pm 2$ & $97 \pm 1$ \\
\hline 120 & $99 \pm 1$ & $96 \pm 2$ & $97 \pm 1$ \\
\hline
\end{tabular}

Tabelle 53: Kumulative Defibrillatorfreiheit (nach Matching)

\subsection{6 Überleben}

Insgesamt verstarben 113 der 700 Patienten der gematchten Population, 18 davon innerhalb von 30 Tagen oder innerhalb des stationären Aufenthaltes nach > 30 Tagen, die Übrigen im Nachbeobachtungszeitraum.

Nach dem Matching gab es einen signifikanten Unterschied im Überleben zum Zeitpunkt des letzten Follow-ups. Gruppe B hatte dabei die größte Anzahl Überlebender (90,9\%) und Gruppe A die kleinste (77,0\%). In diesem Zusammenhang sei an die unterschiedlichen Follow-up-Zeiten der drei Gruppen erinnert. Diese Zeit war bei Gruppe A am längsten mit 130,5 Monaten \pm 46,2 SD und bei Gruppe B am kürzesten mit 47,1 Monaten $\pm 31,8$ SD. Gruppe C lag mit 86,8 Monaten $\pm 32,9$ SD und 18,3\% Verstorbenen zwischen den beiden anderen Gruppen.

\begin{tabular}{|l|l|l|l|l|l|l|}
\hline & $\begin{array}{l}\text { Gruppe A } \\
\mathbf{n = 2 1 3}\end{array}$ & $\begin{array}{l}\text { Gruppe B } \\
\mathbf{n = 2 7 4}\end{array}$ & $\begin{array}{l}\text { Gruppe C } \\
\mathbf{n = 2 1 3}\end{array}$ & $\begin{array}{l}\text { Gesamt } \\
\mathbf{n = 7 0 0}\end{array}$ & $\begin{array}{l}\mathbf{p} \text {-Wert } \\
\text { (Globaltest) }\end{array}$ & $\begin{array}{l}\text { p-Wert } \\
\text { (Paarvergleiche) }\end{array}$ \\
\hline $\begin{array}{l}\text { Überlebensstatus im } \\
\text { letzten Follow-up }\end{array}$ & & & & $\mathbf{0 , 0 0 0 1}$ & $\begin{array}{l}\text { A-B: }<\mathbf{0 , 0 0 1} \\
\text { A-C: } \mathbf{0 , 2 3 1} \\
\text { B-C: } \mathbf{0 , 0 0 6}\end{array}$ \\
\hline lebend & $\begin{array}{l}164 \\
(77,0 \%)\end{array}$ & $\begin{array}{l}249 \\
(90,9 \%)\end{array}$ & $\begin{array}{l}174 \\
(81,7 \%)\end{array}$ & $\begin{array}{l}587 \\
(83,9 \%)\end{array}$ & & \\
\hline verstorben & $\begin{array}{l}49 \\
(23,0 \%)\end{array}$ & $\begin{array}{l}25 \\
(9,1 \%)\end{array}$ & $\begin{array}{l}39 \\
(18,3 \%)\end{array}$ & $\begin{array}{l}113 \\
(16,1 \%)\end{array}$ & & \\
\hline
\end{tabular}

Tabelle 54: Überlebensstatus im letzten Follow-up (nach Matching) 
10.5.6.1 Kaplan-Meier-Schätzung zum Überleben

Die folgende Kaplan-Meier-Kurve gibt Auskunft über Unterschiede beim Überleben in den verschiedenen Gruppen. Die Zeitspanne reichte dabei jeweils vom Datum der Operation bis zum Todesdatum.

Hier sieht man einen deutlichen Unterschied im Vergleich zur Kurve der ungematchten Population. Vor Matching gab es einen signifikanten Unterschied im Überleben, mit signifikant höherer Sterblichkeit bei Gruppe B.

Nach Matching war kein signifikanter Unterschied zwischen den Gruppen mehr nachweisbar.

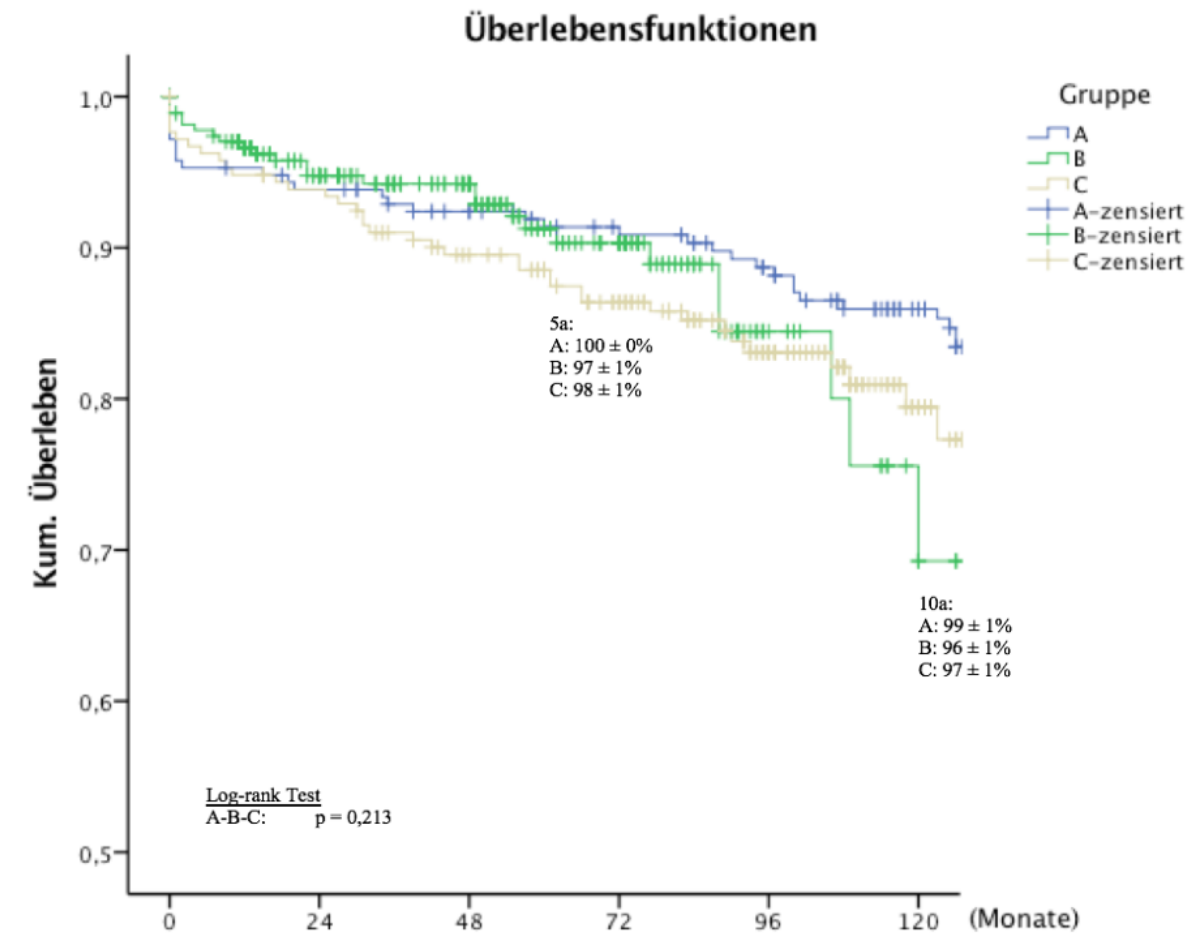

Patienten unter Risiko (n)

$\begin{array}{ccccccc}\text { Gruppe A } & 211 & 193 & 181 & 171 & 155 & 125 \\ \text { Gruppe B } & 241 & 171 & 118 & 56 & 19 & 8 \\ \text { Gruppe C } & 212 & 193 & 171 & 135 & 79 & 29\end{array}$

Abbildung 17: : Kaplan-Meier-Kurve: Überleben (nach Matching) 


\begin{tabular}{|l|l|l|l|}
\hline Zeit in Jahren & \multicolumn{4}{|l|}{ Kumulierter Anteil der Überlebenden \pm SE in \% } \\
\hline & Gruppe A & Gruppe B & Gruppe C \\
\hline 24 & $94 \pm 2$ & $95 \pm 1$ & $94 \pm 2$ \\
\hline 48 & $92 \pm 2$ & $94 \pm 2$ & $89 \pm 2$ \\
\hline 72 & $91 \pm 2$ & $90 \pm 2$ & $86 \pm 2$ \\
\hline 96 & $89 \pm 2$ & $85 \pm 3$ & $83 \pm 3$ \\
\hline 120 & $86 \pm 2$ & $76 \pm 7$ & $80 \pm 3$ \\
\hline
\end{tabular}

Tabelle 55: Kumulierter Anteil Überlebender (nach Matching) 


\section{Multivariate Analysen nach Matching}

Nach dem Matching wurden erneut multivariate Analysen zum Einfluss verschiedener Variablen auf den Ablationserfolg (logistische Regression) und auf die Anzahl der Schrittmacherimplantationen pro Zeit (Cox-Regression) durchgeführt.

Folgende Variablen wurden dabei berücksichtigt:

- Zugang

- Alter

- Pulmonaler Hypertonus

- Präoperative linksatriale Vorhofgröße

- Chirurgische Ablation (nur bei Cox-Regression)

Bei der Variablen Zugang wurde der Zugang B (= POS transseptal) als Referenz festgelegt und der Einfluss von Zugang A (Chitwood) und Zugang $C$ (POS transcaval) im Vergleich zu dieser betrachtet.

\subsection{Logistische Regression: Ablationserfolg bei Entlassung und im letzten Follow-up}

Als Ablationserfolg wurde ein Sinusrhythmus nach erfolgter Ablation gewertet. In der Auswertung wurde Vorhofflimmern als unabhängige Variable festgelegt. Ein $\mathrm{OR}<1$ verringerte also das Risiko für Vorhofflimmern, ein $\mathrm{OR}=0$ hatte keinen Einfluss und ein OR > 1 erhöhte das Risiko für Vorhofflimmern nach Ablation.

Insgesamt änderten sich die Zahlen bei der gematchten Analyse nur gering gegenüber den ungematchten, so dass die Kernaussagen die gleichen blieben. Bei Anwendung der rechtsseitigen anterolateralen Minithorakotomie unter Verwendung der Chitwood-Klemme war der Ablationserfolg im Vergleich zur partiellen oberen Sternotomie über den transseptalen Zugang signifikant größer. 
Einen weiteren signifikanten Einfluss auf den Ablationserfolg zeigte das Lebensalter: Je höher das Alter, umso geringer der Ablationserfolg. Ein Einfluss der präoperativen linksatrialen Vorhofgröße konnte nach Matching zum postoperativen Zeitpunkt nicht mehr nachgewiesen werden.

\subsection{Logistische Regression: Ablationserfolg bei Entlassung}

\begin{tabular}{|l|l|l|l|l|}
\hline Kovariaten & p-Wert & Odds Ratio (OR) & \multicolumn{2}{l|}{$\mathbf{9 5 , 0} \%$-Konfidenzintervall } \\
\hline Zugang A vs. Zugang B & 0,039 & 0,156 & 0,027 & 0,914 \\
\hline Zugang C vs. Zugang B & 0,394 & 0,531 & 0,124 & 2,275 \\
\hline Alter & 0,006 & 1,113 & 1,031 & 1,201 \\
\hline Pulmonaler Hypertonus & 0,616 & 1,393 & 0,381 & 5,094 \\
\hline LA-Größe & 0,088 & 0,507 & 0,232 & 1,106 \\
\hline
\end{tabular}

Tabelle 56: Logistische Regression: Einflussfaktoren auf den Ablationserfolg bei Entlassung (nach Matching)

\subsection{Logistische Regression: Ablationserfolg im Last Follow-up}

In der Langzeitbeobachtung hatte weiterhin der transcavale im Vergleich zum transseptalen Zugang über partielle obere Sternotomie einen größeren Ablationserfolg, also ein signifikant geringeres Risiko für Vorhofflimmern. Das Alter war auch hier ein negativer Einfluss. Die Vorhofgröße blieb der stärkste Prädiktor für den Ablationserfolg. Je größer der präoperative LA-Durchmesser, umso höher das Risiko für Vorhofflimmern nach Ablation.

\begin{tabular}{|l|l|l|l|l|}
\hline Kovariaten & p-Wert & Odds Ratio (OR) & \multicolumn{2}{|l|}{$\mathbf{9 5 , 0} \%$-Konfidenzintervall } \\
\hline Zugang A vs. Zugang B & 0,959 & 0,959 & 0,196 & 4,679 \\
\hline Zugang C vs. Zugang B & 0,011 & 0,093 & 0,015 & 0,585 \\
\hline Alter & 0,001 & 1,148 & 1,059 & 1,245 \\
\hline Pulmonaler Hypertonus & 0,858 & 1,141 & 0,27 & 4,832 \\
\hline LA-Größe & 0,026 & 3,186 & 1,149 & 8,836 \\
\hline
\end{tabular}

Tabelle 57: Logistische Regression: Einflussfaktoren auf den Ablationserfolg im letzten Follow-up (nach Matching) 


\subsection{Cox-Regression: Einfluss auf die Schrittmacherhäufigkeit}

In der gematchten Population konnte ein positiver Einfluss des Zugangs über die rechtsseitige anterolaterale Minithorakotomie unter Verwendung der Chitwood-Klemme auf die Anzahl notwendiger Schrittmacherimplantationen nicht mehr nachgewiesen werden.

Die partielle obere Sternotomie mit transcavalem Zugang hingegen erhöhte auch nach Matching signifikant die Anzahl an Schrittmacherimplantationen. Das Alter blieb weiterhin ein negativer Einfluss. Den stärksten Einfluss auf die Schrittmacherinzidenz hatte die Ablation. Sie erhöhte das Risiko für eine Schrittmacherimplantation stark.

\begin{tabular}{|l|l|l|l|l|}
\hline Kovariaten & $\mathbf{p}$-Wert & Hazard Ratio (HR) & \multicolumn{2}{|l|}{$\mathbf{9 5 , 0} \%$-Konfidenzintervall } \\
\hline Zugang A vs. Zugang B & 0,1 & 0,415 & 0,146 & 1,183 \\
\hline Zugang C vs. Zugang B & 0,034 & 2,275 & 1,065 & 4,863 \\
\hline Alter & 0,001 & 1,056 & 1,024 & 1,09 \\
\hline Pulmonaler Hypertonus & 0,449 & 0,771 & 0,394 & 1,509 \\
\hline LA-Größe & 0,463 & 0,872 & 0,606 & 1,256 \\
\hline Ablation & $<0,0001$ & 4,244 & 2,166 & 8,315 \\
\hline
\end{tabular}

Tabelle 58: Cox-Regression: Einfluss auf die Schrittmacherhäufigkeit (nach Matching) 


\section{Diskussion}

In der vorliegenden Studie wurden 1206 Patienten untersucht, die sich zwischen Januar 1998 und Dezember 2015 an der Klinik für Thorax-, Herz- und thorakale Gefäßchirurgie der Universitätsklinik Frankfurt am Main einem minimalinvasiven Mitralklappeneingriff unterzogen hatten. Dabei erfolgte die Einteilung der Patienten je nach Zugangsweg in Gruppe A ( $n=300$, anterolaterale Minithorakotomie mittels Chitwood-Klemme mit linksatrialem Zugang), Gruppe B ( $n=687$, partielle obere Sternotomie mit superiorem transseptalem Zugang) und Gruppe C ( $n=219$, partielle obere Sternotomie mit transcavalem Zugang). Es wurden innerklinische Daten sowie externe Untersuchungsbefunde und ein standardisierter Fragebogen ausgewertet. Ziel der Studie war es, Unterschiede dieser drei Zugangswege im Hinblick auf die Inzidenz von Vorhofflimmern bei präoperativ Gesunden, den Erfolg einer perioperativ durchgeführten chirurgischen Ablation sowie die postoperative Notwendigkeit eines Herzschrittmachers und die Überlebensraten aufzuzeigen. Nach Auswertung der Originaldaten erfolgte eine erneute Auswertung nach Propensity Matching, um die gezeigten Ergebnisse ohne präoperative Unterschiede zu verifizieren. Nach Egalisierung der signifikanten Differenzen in den prä- und perioperativen Daten der drei Gruppen in den Punkten Alter, Geschlecht, pulmonaler Hypertonus, zusätzliche Klappeneingriffe (meist TKR), Vorhofflimmern, Schrittmacher und Voroperationen, konnten die Ergebnisse besser auf den Zugangsweg zurückgeführt werden.

Ein Follow-up konnte bei 1050 der 1141 Überlebenden (92\%) generiert werden. Die Follow-up-Zeiten waren aufgrund der in verschiedenen Zeiträumen bevorzugt genutzten Zugangswege und des langen Einschlusszeitraumes von 17 Jahren sehr unterschiedlich. Zu Beginn des Beobachtungszeitraums waren hauptsächlich Eingriffe über die anterolaterale Minithorakotomie mittlels Chitwood-Klemme gemacht worden, gefolgt von der partiellen oberen Sternotomie mit transseptalem und transcavalem Zugang. 
Bei Gruppe A bestand daher die längste und variabelste Follow-up-Zeit mit 128,7 \pm 46,8 SD Monaten (Min. 2, Max. 217 Monate). Bei Gruppe B war die mittlere Follow-up-Zeit 49,4 Monate $\pm 33,0$ SD und Gruppe C hatte 86,4 Monate $\pm 32,9$ SD. Insgesamt ergab sich eine mittlere Follow-up-Zeit von $76 \pm 49,4$ SD Monaten und eine kumulative Follow-up-Zeit von 6560 Patientenjahren. Nach Matching verringerte sich die kumulative Follow-up-Zeit auf 4419 Patientenjahre. Die mittlere Follow-up-Zeit verlängerte sich auf 83,8 \pm 50,4 Monate, da durch das Matching im Verhältnis die meisten Patienten aus Gruppe B (kürzeste FU-Zeiten) wegfielen. Das Verhältnis zwischen den Gruppen bezüglich der mittleren FU-Zeit blieb gleich.

\subsection{Inzidenz von Vorhofflimmern bei präoperativem Sinusrhythmus}

Vorhofflimmern tritt häufig nach herzchirurgischen Eingriffen, insbesondere nach Mitralklappenoperationen auf. ${ }^{44,} 45$ Die Inzidenz und der Einfluss des verwendeten Zugangsweges, insbesondere der atrialen Inzision, sind noch nicht hinreichend geklärt.

In unserer Studie hatten 20,7\% der 653 Patienten mit präoperativem Sinusrhythmus im letzten Follow-up Vorhofflimmern. Inkludiert sind jene Patienten, die bereits postoperativ, d. h. zum Zeitpunkt der Entlassung, Vorhofflimmern hatten (von denen manche im Verlauf spontan oder interventionell konvertierten und somit wegfielen), sowie auch jene, die im Follow-up Vorhofflimmern bekamen.

In Gruppe B war sowohl postoperativ (12,8\%) als auch im letzten Follow-up $(23,5 \%)$ die größte Zahl an Patienten mit neu aufgetretenem Vorhofflimmern zu verzeichnen. Gruppe A zeigte postoperativ das beste Ergebnis mit nur 7,3\% Vorhofflimmern und Gruppe $\mathrm{C}$ zeigte sich in der Langzeitbeobachtung beim letzten Follow-up mit nur 17,0 \% Vorhofflimmern am besten. Es bestand jedoch kein statistisch signifikanter Unterschied zwischen den Gruppen. 
Anhand der kumulativen Follow-up-Zeit konnte eine Inzidenz für neu aufgetretenes Vorhofflimmern im letzten Follow-up von 1,0 \% für Gruppe A, 3,3 \% für Gruppe B und 1,7 \% für Gruppe C pro Patientenjahr errechnet werden. Diese Ergebnisse blieben nach Matching unverändert.

Es gibt eine Vielzahl von Studien, in denen der Zugangsweg zur Mitralklappe, insbesondere die Art der Vorhofinzision, als entscheidend im Hinblick auf Rhythmusstörungen und Schrittmacherimplantationen erscheint. Verglichen werden vielfach die Linksatriotomie (entsprechend unserer Gruppe A) mit der (superioren) transseptalen Inzision (entsprechend unserer Gruppe B). Der transcavale Zugang (Gruppe $\mathrm{C}$ ) ist in der diesbezüglichen Literatur nicht vertreten.

Zwar zeigt unsere Studie keinen signifikanten Unterschied im Neuauftreten von postoperativem Vorhofflimmern, jedoch konnte bei Gruppe B deutlich häufiger VHF festgestellt werden als bei den Gruppen A und C.

Dieser Unterschied erschien in anderen Studien mit statistischer Signifikanz. Rezahosseini et al. (2014) zeigten ermittelten in einer großen retrospektiven Studie nach Propensity Matching bzgl. ähnlicher Einflussgrößen wie in unserer Studie (LA-Größe, präoperatives VHF, Alter etc.) ein signifikant häufigeres Auftreten von neuem postoperativem Vorhofflimmern beim transseptalen im Vergleich zum linksatrialen Zugang. ${ }^{74}$ Lukac et al. (2006) untersuchten postoperative atriale Arrhythmien in einer Studie mit 213 Patienten und verglichen den superioren transseptalen mit dem linksatrialen Zugang. Der transseptale Zugang sowie ein Lebensalter $>65$ Jahre und das männliche Geschlecht stellten unabhängige Prädiktoren für atriale Tachykardien dar. Für Vorhofflimmern war das Alter $>60$ Jahre der einzige unabhängige Prädiktor.

Es zeigte sich in der transseptalen Gruppe signifikant mehr Vorhofflimmern als atriale Tachykardie, in der linksatrialen Gruppe waren beide gleich vertreten. ${ }^{75}$ 
Bei Utley et al. (1995) in einer Studie mit relativ kleiner Fallzahl $(n=149)$ wurden der transseptale, der superior transseptale und der linksatriale Zugang verglichen. Hier hatten die Patienten mit linksatrialem Zugang (6/44, 14 \%) ebenfalls deutlich seltener neu aufgetretenes postperatives Vorhofflimmern als die mit superior transseptalem Zugang (6/28, $21 \%)$. Keinen Fall von Vorhofflimmern gab es hier beim rein transseptalen Zugang (0/13). Signifikant waren die gezeigten Unterschiede jedoch auch hier nicht. ${ }^{76}$

Obwohl der transcavale Zugang dem linksatrialen in Bezug auf die Schnittlinien im linken Vorhof sehr ähnlich ist, zeigt sich hier ein häufigeres Auftreten von Vorhofflimmern.

Interessant ist auch eine sehr große retrospektive Studie von Gialdini et al. (2014) zu perioperativem Vorhofflimmern und Schlaganfallrisiko.

Ausgeschlossen aus der Studie wurden Patienten, die präoperativ jemals Vorhofflimmern oder cerebrovaskuläre Ereignisse gehabt hatten oder erst nach Entlassung Vorhofflimmern bekamen. Hier zeigte sich in der Cox-Regression eine Assoziation von perioperativem Vorhofflimmern (sowohl nach Herzchirurgie als auch nach anderen operativen Eingriffen) mit einem erhöhten Langzeit-Schlaganfallrisiko. Dieser Zusammenhang blieb auch nach Berücksichtigung potenzieller Einflussfaktoren (Alter, Geschlecht, präoperatives Schlaganfallrisiko) bestehen. ${ }^{77}$

Dies unterstreicht die Relevanz des Bemühens, den Ursachen von perioperativem Vorhofflimmern auf den Grund zu gehen und das Auftreten möglichst zu vermeiden.

\subsection{Inzidenz von Schrittmacherimplantationen}

Im Beobachtungszeitraum (perioperativ bis zum letzten Follow-up) wurde bei 140 Patienten (11,6 \%) von den gesamten 1206 Patienten ein Schrittmacher implantiert. Davon erfolgten die signifikant meisten Schrittmacherimplantationen in Gruppe C mit 16,0\%, die Wenigsten in Gruppe A mit 7,3\%. 
Von der Gesamtzahl der Patienten mit Schrittmachern waren 33,5 \% zuvor chirurgisch abladiert worden. Auch nach Ablation zeigte Gruppe $\mathrm{C}$ die höchste (3,9 \%) und Gruppe A die geringste (2,7\%) Schrittmacheranzahl. Eine mögliche Erklärung für dieses Ergebnis wären die sich von den anderen beiden Zugangswegen unterscheidenden Ablationslinien beim transcavalen Zugang (Inzision oder Ablation des rechten Vorhofs auf der Strecke von der Vena cava inferior bis zur Vena cava superior).

Betrachtet man jedoch die Schrittmacheranzahl bei den nicht abladierten Patienten (Gruppe C 11,1\%, Gruppe B 10,7\% und Gruppe A 5,8 \%), so scheint die Schrittmacherhäufigkeit unabhängig von der Ablation in beträchtlichem Ausmaß von der Vorhofinzision bestimmt zu werden. In Gruppe $B$ und $C$ ist die Schrittmacheranzahl nahezu doppelt so groß wie in Gruppe A.

Im Verlauf konnte in der Zeit nach der Entlassung ein deutlicher Anstieg der Schrittmacheranzahl bis zum Zeitpunkt des letzten Follow-ups aufgezeigt werden.

In Gruppe C war dieser Anstieg mit 9,5\% am deutlichsten und in Gruppe A mit 7,7\% am geringsten.

Mit Hilfe der kumulativen Follow-up-Zeit konnte eine Inzidenz für Schrittmacherimplantationen von 0,8 \% für Gruppe A, 3,1 \% für Gruppe B und 2,3 \% für Gruppe $C$ pro Patientenjahr errechnet werden. Diese veränderte sich nach Matching nur bei Gruppe B auf 2,2 \%, so dass Gruppe C auch hier am schlechtesten abschnitt.

Die veränderten Ergebnisse nach Matching zu Gunsten von Gruppe B lassen darauf schließen, dass die zusätzlichen Klappeneingriffe, die in Gruppe B in Form der Trikuspidalklappenrekonstruktion häufiger waren, sowie auch die präoperativ höhere Zahl vorhandener Schrittmacher die Ergebnisse beeinflussten.

Die unterschiedliche Follow-up-Zeit konnte mittels Kaplan-Meier-Schätzer herausgerechnet werden. 
An der Kaplan-Meier-Kurve wird deutlich sichtbar, dass Gruppe A gegenüber Gruppe B und C in Bezug auf die Schrittmacherfreiheit signifikant überlegen war. Nach 5 Jahren waren bei Gruppe A noch $98 \pm 1 \%$ der Patienten ohne Schrittmacher. In Gruppe B waren es $89 \pm 2 \%$, in Gruppe C $87 \pm 2 \%$. Nach 10 Jahren waren es in Gruppe A immer noch $92 \pm 2 \%$, in Gruppe B $87 \pm 3 \%$ und in Gruppe C $78 \pm 4 \%$. Sichtbar wird auch, dass die Zahl der perioperativen Schrittmacherimplantationen in Gruppe C und B deutlich höher war als bei Gruppe A mit nur einer einzigen Schrittmacherimplantation.

Wenngleich die Zahl der Schrittmacher-Neuimplantationen nach Matching im Beobachtungszeitraum nach Entlassung bei Gruppe A höher war als vor dem Matching, sind doch die absoluten Zahlen perioperativ, postoperativ und im letzten Follow-up im Vergleich zu den anderen Gruppen am geringsten.

Zusammenfassend lässt sich festhalten, dass in unserer Studie Gruppe A den Gruppen B und C in Bezug auf die Schrittmacherinzidenz überlegen ist, wobei Gruppe C schlechter abschneidet als Gruppe B.

Dass aufgrund der räumlichen Nähe durch Eröffnen des rechten Vorhofs und transseptale Inzision ein hohes Risiko für eine Beschädigung der

Sinusknotenarterie mit Ischämie der Sinusknotenregion oder Beschädigung des Sinusknotens selbst besteht, ist bereits beschrieben worden. ${ }^{78}$ Dass jedoch beim transcavalen Zugang diesbezüglich Probleme auftreten, ist unerwartet. Da sowohl der rechtslaterale Zugang als auch die partielle obere Sternotomie mit transcavalem Zugang unter Verschonung des rechten Vorhofs über die interatriale Grube und das Dach des linken Vorhofs ohne transseptale Inzision zur Mitralklappe führen, hätte man ähnliche Ergebnisse der Gruppen A und C erwartet. Es besteht jedoch ein signifikanter Unterschied zwischen den Gruppen A und C sowie B und C, nicht jedoch zwischen A und B.

Zum transcavalen Zugang sowie dessen Einfluss auf den Herzrhythmus oder die postoperative Schrittmacherinzidenz gibt es bislang keine Beschreibungen in der Literatur. 
Der transseptale Zugang bringt den Vorteil der besten Sicht auf die Mitralklappe sowie die Möglichkeit der zusätzlichen Trikuspidalklappenrekonstruktion mit sich. Vielfach wurden auch hier die Zugangswege der Gruppe A und B verglichen und die Frage nach postoperativen Schrittmacherinzidenzen diskutiert.

In unserer Studie gab es in Gruppe B gemäß der ursprünglichen Auswertung insgesamt deutlich mehr Schrittmacherimplantationen als in Gruppe A, jedoch ohne signifikanten Unterschied. Nach Matching bestand mit 8,9 \% und 8,5\% kein wesentlicher Unterschied mehr.

Dies entspricht den Ergebnissen der o. g. retrospektiven Studie von Rezahosseini et al. (2014), in der nach Propensity Matching ebenfalls keine signifikant erhöhte Schrittmacherrate in der transseptalen im Vergleich zur linksatrialen Gruppe gezeigt werden konnte. ${ }^{74}$

In einer aktuellen Studie ( $n=469)$ von Boulemden et al. (2019) wurden die atrialen Zugangswege transseptal, superior transseptal und linksatrial mit der Frage nach dem Einfluss auf postoperative Schrittmacherimplantationen und dem Effekt der Sinusknotenarterie auf die Schrittmacherimplantation untersucht. Dabei konnte kein Einfluss des atrialen Zugangsweges auf die postoperative Schrittmacherinzidenz festgestellt werden. In der univariaten Analyse waren nur Alter und zusätzliche Klappeneingriffe (TKR) signifikante Prädiktoren für postoperative Schrittmacherimplantationen, in der multivariaten Analyse nur das Alter. ${ }^{79}$

Auch Aydin et al. (2014) kamen zu dem Ergebnis, dass der superiore transseptale Zugang nicht zu signifikant erhöhter Schrittmacherinzidenz oder erhöhter Mortalität führt. ${ }^{80}$

Nienaber et al. (2006) beschrieben den minitransseptalen Zugang, bei dem die Inzision nicht bis zum Vorhofdach hochgezogen wird. Dieser war in der multivariaten Analyse kein unabhängiger Prädiktor für Schrittmacher oder für Rhythmusveränderungen wie den junktionalen Ersatzrhythmus und Vorhofflimmern. 
Jedoch zeigte sich bei der vorherigen univariaten Auswertung ein vermehrtes Auftreten eben dieser Faktoren (wie auch in unserer Studie im postoperativen Status vor Matching), die auf Resternotomien und zusätzliche Trikuspidalklappeneingriffe entsprechend unserer Gruppe B zurückgeführt werden konnten. ${ }^{81}$

In der o. g. von Utley at al. (1995) durchgeführten prospektiven Studie erwies sich in der multiplen Regression die Art der atrialen Inzision nicht als Prädiktor für die permanente Schrittmacherimplantation. ${ }^{76}$ Lukac et al. (2007) zeigten jedoch in ihrer retrospektiven Studie an 577 Patienten, dass bei der partiellen oberen Sternotomie mit superiorem transseptalen Zugang ein signifikant höheres Risiko (11,3\%) einer Sinusknotendysfunktion mit konsekutiver Inzidenz von Schrittmacherimplantationen besteht, im Vergleich zur anterolateralen Minithorakotomie mit linksatrialem Zugang (6,3\%).

Es gab keinen Unterschied der Gruppen bezüglich AV-KnotenÜberleitungsstörungen als Ursache für Schrittmacherimplantationen. In der bivariaten Analyse zeigte sich der transseptale Zugang als unabhängiger Risikofaktor für Schrittmacherimplantationen. ${ }^{33}$ In der prospektiven Studie von Kumar et al. (1995) wurde der frühe postoperative Rhythmus verglichen. In der superioren transseptalen Gruppe hatten $38 \%$ einen junktionalen Ersatzrhythmus im Vergleich zu $13 \%$ bei der linksatrialen Gruppe. ${ }^{82}$

Zusammenfassend wurde gezeigt, dass insbesondere die superiore transseptale Inzision (wie in unserer Studie angewandt) postoperative Rhythmusstörungen und Schrittmacherimplantationen aufgrund einer Sinusknotendysfunkton begünstigen kann. Auch wenn die Ergebnisse wie in unserer Studie häufig nicht signifikant sind, sind die Zahlen doch meist höher als bei Gruppe A. 
Die variierenden Ergebnisse der Studien sind möglicherweise auf die nicht exakt identischen transseptalen Inzisionen im Sinne der Länge und der Positionierung des Schnittes zurückzuführen. Dies ist in verschiedenen Studien unterschiedlich beschrieben und nicht eindeutig abzugrenzen. Des Weiteren ist die Tatsache zu berücksichtigen, dass der superior transseptale Zugang in den Studien häufig bei Patienten verwendet wurde, die einen kleinen linken Vorhof hatten, reoperiert wurden und bei denen die Trikuspidalklappe zusätzlich operiert wurde.

Außerdem spielt natürlich die individuelle Durchführung durch den Chirurgen immer eine Rolle. Selbst bei ein und demselben Operateur unterliegt diese sicherlich gewissen Schwankungen.

Auch wenn die Studienlage nicht eindeutig ist, wurden Alternativen bzw. Modifizierungen erforscht, um das Risiko der Verletzung der Sinusknotenarterie zu verringern.

Suzuki et al. (2011) stellten eine Technik des superioren transseptalen Zugangs vor, bei dem die Sinusknotenarterie effektiv geschont und die Inzidenz einer postoperativen Sinusknotendysfunktion vermindert werden kann. ${ }^{83}$ Hierbei wurde das rechte Atrium parallel zur interatrialen Grube eröffnet und die Inzision superior zur Sinusknotenarterie ausgeweitet. Diese wurde dann etwa $2 \mathrm{~cm}$ der Länge nach präpariert, mit einem elastischen Band umschlungen und so aus dem Gefahrengebiet separiert. Anschließend erfolgte die Exposition der Mitralklappe wie beim ursprünglichen superioren transseptalen Zugang. Des Weiteren wurde von Legare et al. (2003) ein alternativer Zugang über das Vorhofdach erprobt und dieser ergab ein vergleichbares Outcome zu superior transseptaler und linksatrialer inzision. ${ }^{84}$

In einer Studie von 2009 mit 42 Patienten konnte das Vermeiden der Beschädigung der Sinusknotenarterie bei diesem Zugang gezeigt werden; 88 \% der Patienten waren bei Entlassung im Sinusrhythmus. ${ }^{85}$ 
Erstmals beschrieben wurde dieser Zugang von Meyer und Kollegen (1965) sowie Saksena und Kollegen (1971).

Warum der transcavale Zugang, wie in unserer Studie gezeigt, mit einer noch höheren Inzidenz für Schrittmacherimplantationen einhergeht als Gruppe B und sich in diesem Punkt signifikant von Gruppe A unterscheidet, kann aus der vorhandenen Literatur nicht erklärt werden. Ein möglicher Erklärungsansatz ist, dass bei diesem Zugang am meisten Gewebe um das Sinusknotenareal mobilisiert wird.

Dies gibt Anlass für weitere Untersuchungen.

\subsection{Ablation und Schrittmacherinzidenz}

Insgesamt erhielten 22 der 234 abladierten Patienten (9,4\%) perioperativ (innerhalb von 14 Tagen) einen Schrittmacher. Von den 972 nicht abladierten Patienten erhielten perioperativ 25 (2,6\%) einen Schrittmacher.

Die signifikant meisten Schrittmacher nach Ablation gab es in Gruppe $C$ mit $25 \%$, in Gruppe A gab es keinen Fall. Auch nach Matching blieb dies im Wesentlichen unverändert.

Auf den ganzen Beobachtungszeitraum bezogen, $d$. h. perioperativ und im gesamten Follow-up, erhielten 140 Patienten einen Schrittmacher, davon 47 nach Ablation. Das bedeutet: 20 \% (47/234) der abladierten im Gegensatz zu 9,5\% (93/972) der nicht abladierten Patienten bekamen einen Schrittmacher. Gruppe $C$ schnitt hier wie auch bei der gesamten Schrittmacherinzidenz am schlechtesten ab und Gruppe A am besten. Nach Matching blieb Gruppe C weiterhin am schlechtesten, aber Gruppe B anstelle von A hatte hier das beste Ergebnis.

In unserer wie auch in vorherigen Studien anderer Autoren konnte gezeigt werden, dass die additive chirurgische Ablation zwar effektiv ist, aber die Schrittmacherinzidenz deutlich steigert. ${ }^{72,73}$ 
Vergleichende Studien bezüglich des zusätzlichen Einflusses des

Zugangsweges, insbesondere der atrialen Inzision, gibt es auch hierzu nicht. In einer randomisiert kontrollierten Studie $(n=260)$ von Gillinov et al. (2015) benötigten signifikant mehr Patienten, die zuvor abladiert worden waren, einen Schrittmacher als die nicht Abladierten (21.5 vs. 8.1 pro 100 Patientenjahre). Es gab keinen signifikanten Unterschied bzgl. der Mortalität und innerhalb der Abladierten keinen Unterschied zwischen Pulmonalvenenisolation und biatrialem Maze. ${ }^{72}$ Nach Zugangswegen wurde nicht unterschieden. In einer 2017 veröffentlichten Studie konnte nach Propensity Matching eine Assoziation von chirurgischer Ablation mit einer reduzierten 30-Tage-Mortalität und reduziertem Schlaganfallrisiko ermittelt werden. Das Risiko für Nierenversagen und Schrittmacherbedarf bei den Patienten stieg jedoch an. ${ }^{86}$ De Rose et al. (2019) zeigten an 232 Patienten mit Ablation (Pulmonalvenenisolation oder biatrialer Maze), dass Ablation, Mehrfachklappenchirurgie und eine Herzinsuffizienz NYHA IV jeweils mit Schrittmacherimplantationen assoziiert sind. Außerdem zeigte sich eine signifikant erhöhte 1-Jahres-Mortalität bei Patienten mit implantiertem Schrittmacher nach Mitralklappenchirurgie. ${ }^{87}$

\subsection{Ablationserfolg bei präoperativ bestehendem Vorhofflimmern}

In der ursprünglichen Auswertung zeigte sich hinsichtlich des Ablationserfolges zum Zeitpunkt der Entlassung ein signifikanter Unterschied zwischen den Gruppen A und B.

Insgesamt konvertierten nach Ablation 63,3\% der Patienten in den Sinusrhythmus. Den signifikant größten Ablationserfolg konnte Gruppe A verzeichnen. Zum Zeitpunkt der Entlassung waren hier $86,8 \%$ der Patienten in den Sinusrhythmus konvertiert.

Das signifikant schlechteste Ablationsergebnis zeigte sich in Gruppe B mit nur $50,8 \%$ Konversion. 
Nach Matching blieben diese Ergebnisse signifikant, so dass die geringe Erfolgsrate von Gruppe B hier nicht auf Kombinationseingriffe, Alter oder präoperative LA-Größe zurückzuführen war. Im letzten Follow-up waren bei Gruppe A nur noch 47,1 \% der Patienten im Sinusrhythmus, so dass sich von Entlassung bis zum letzten Follow-up eine Vorhofflimmern-Rezidivrate von 39,7 \% errechnen ließ. Gruppe C zeigte im letzten Follow-up mit 59,0\% Sinusrhythmus das beste Ergebnis. Dieser Unterschied war signifikant.

Nach Matching zeigte sich im letzten Follow-up kein signifikanter Unterschied zwischen den Gruppen mehr, wobei weiterhin $C$ das beste und A das schlechteste Ergebnis hatten. Dass Gruppe A die höchste VorhofflimmernRezidivrate (51,5\%) im letzten Follow-up hatte, kann durch die mit 128,7 $\pm 46,8$ SD Monaten längste mittlere Follow-up-Zeit von Gruppe A erklärt werden. Bei Gruppe B waren dies nur 49,4 33 SD Monate und es ist offensichtlich, dass Gruppe A über diesen langen Zeitraum mehr Rezidive entwickeln konnte. Die Ergebnisse nach Relativierung dieser Laufzeitunterschiede mittels Berechnung der Inzidenzen anhand der kumulativen Follow-up-Zeit bestätigen dies. Hier zeigt sich die größte Inzidenz pro Patientenjahr für ein Vorhofflimmerrezidiv nach Ablation in Gruppe B (2,9\%) und nahezu identische Inzidenzen bei den Gruppen $A$ und $C$ (1,0\% und 1,1\% pro Patientenjahr). Nach Matching sank die Inzidenz in Gruppe B auf 1,6\%, in den anderen beiden Gruppen blieb sie gleich.

Bei etwa 30 bis $50 \%$ der Patienten, die sich einer Mitralklappenoperation unterziehen, besteht Vorhofflimmern, das mit einem erhöhten Schlaganfallrisiko und geringerer Lebenserwartung assoziiert ist. ${ }^{88}$ Von großem Interessesind daher die Effizienz und das Risiko einer additiv durchgeführten chirurgischen Ablation.

Dass die chirurgische Ablation ein effizientes Werkzeug ist, um Vorhofflimmern zu therapieren, konnte unter anderem von Raanani et al. (2000) gezeigt werden. 
Diese Arbeitsgruppe untersuchte die Maze-Prozedur, additiv durchgeführt zur Mitralklappenchirurgie. Die Ablation zeigte sich effektiv im Auslöschen von Vorhofflimmern und reduzierte das Risiko für thrombembolische Ereignisse. Die Mortalität der Mitralklappenchirurgie wurde dadurch nicht erhöht. ${ }^{89}$ Interessant ist, ob auch die Art der atrialen Inzision einen Einfluss auf den Ablationserfolg hat. Vergleichende Studien über Ablation bei diesen drei Zugangswegen gibt es in der Literatur nicht. Studien zu additiver Ablation, bei denen jedoch häufig der Zugangsweg nicht erwähnt oder nicht dahingehend differenziert wird, sind vorhanden.

In unserer Studie konnte Gruppe A in der postoperativen Betrachtung ein signifikant besseres Ergebnis erreichen als Gruppe B. In der Langzeitbetrachtung zeigte sich Gruppe $\mathrm{C}$ am besten. Nach Matching war der Unterschied zwar nicht mehr signifikant, das Verhältnis zwischen den Gruppen blieb aber gleich.

In Gruppe $A$ und $C$ war mittels Zugang über die interatriale Grube ein relativ einfaches komplettes Abtrennen der Pulmonalvenen möglich.

Das schlechte Abschneiden von Gruppe B könnte mit der komplizierteren Durchführung der Maze-Prozedur durch den transseptalen Zugang verbunden sein, die mit den Jahren kontinuierlich verbessert wurde. In unserer Studie wurde 2004 mit dem superioren transseptalen Zugang begonnen.

Doukas et al. konnten in einer randomisierten Studie die Effektivität der zusätzlichen Ablation in der Mitralklappenchirurgie bei Patienten mit präoperativem Vorhofflimmern bestätigen. Verwendet wurden der linksatriale und der transseptale Zugang, es erfolgte jedoch keine Differenzierung. Bei Entlassung hatten 53,3\% und nach 12 Monaten 44,4\% der abladierten Patienten einen Sinusrhythmus, während es in der nicht abladierten Gruppe nur 4,5\% waren. Die postoperativen Komplikationen und die Mortalität unterschieden sich kaum. ${ }^{90} \mathrm{Im}$ Vergleich dazu war in unserer Studie die Sinuskonversion von Gruppe A und B zum Zeitpunkt der Entlassung mit 61,7 \% besser. 
Gillinov et al. stellten 2007 ihre erfolgversprechende Methode der biatrialen Ablation über den superioren transseptalen Zugang vor. ${ }^{88}$ Auch bei Benussi et al. (2010), die die bipolare Radiofrequenz Maze-Procedur als Soloeingriff durch einen transseptalen Zugang als gut praktikabel beschrieben, gab es keine Komplikationen und die Erfolgsrate nach $11 \pm 6$ SD Monaten lag bei $80 \% .{ }^{91}$ In einer prospektiven Studie von Oueida et al. (2014) an 52 Patienten kam es nach linksatrialer Ablation zu 84 \% Sinuskonversion bei Entlassung und nach 12 Monaten waren 88,5 \% der Patienten im Sinusrhythmus. Als Zugangswege wurden hier die Linksatriotomie und der superiore transseptale Zugang verwendet, jedoch nicht verglichen. Die postoperative Konversionsrate war hier verglichen mit der Gesamtzahl von Sinuskonversionen der Gruppen A und B unserer Studie $(61,7 \%)$ sehr viel höher. ${ }^{92}$ Dies liegt möglicherweise an einer bis dato verbesserten Durchführbarkeit dieser Methode.

Im Vergleich zu einer Studie von Tiwari et al. (2016) war unser Ergebnis von Gruppe A mit $86 \%$ Sinusrhythmus bei Entlassung und 47,1\% nach einer mittleren Follow-up-Zeit von 128,7 \pm 46,8 SD Monaten sehr viel besser. In deren Studie über Ablation via rechtsseitiger Minithorakotomie waren bei Entlassung 57,3\% der Patienten in den Sinusrhythmus konvertiert. Nach einem mittleren Follow-up von 21,6 $\pm 10,1$ Monaten waren $63 \%$ im Sinusrhythmus. Diese Zahl ist zwar deutlich besser als in unserer Studie, jedoch war die mittlere Follow-up-Zeit auch - verglichen mit unserer - um mehr als das Zehnfache kürzer.

Auch verglichen mit einer von Mohr et al. (2002) durchgeführten Studie schnitt unsere Gruppe A mit 86,8 \% Sinusrhythmus bei Entlassung gegenüber deren äquivalenter Gruppe mit 76,8\%, deutlich besser ab. Eine ebenfalls untersuchte Gruppe mit isolierter Ablation über einen minimalinvasiven Zugang hatte zu allen Zeitpunkten $>90 \%$ Sinuskonversion. ${ }^{93}$

Bei Krakor et al. (2010) bestand nach 6 Monaten bei $80 \%$ der über den rechtslateralen Zugang an der Mitralklappe operierten und abladierten 
Patienten ein Sinusrhythmus. Zwischen epi- und endokardialer Ablation gab es keinen Unterschied in der Erfolgsrate. ${ }^{94}$

Zusammenfassend lässt sich feststellen, dass in unserer Studie Gruppe A die postoperativ beste Konversionsrate lieferte, allerdings mit höchster Rezidivrate bei längster Follow-up-Zeit.

In der langfristigen Beobachtung scheint Gruppe $\mathrm{C}$ die besten Ergebnisse zu bringen. Für eine zielführende Aussage bedarf es weiterer Studien, die diese drei Zugangswege bei gleicher Ablationsdurchführung und einheitlichen Followup-Zeiten vergleichen.

Des Weiteren gibt es andere Faktoren wie z. B. Alkoholkonsum, der laut einer jüngst veröffentlichten Studie maßgeblich ursächlich für ein Vorhofflimmerrezidiv ist. ${ }^{95}$

\section{5 Überleben}

Nach initialem Vergleich der drei Gruppen zeigte sich eine erhöhte Frühsterblichkeit (innerhalb von 30 Tagen und intrahospital > 30 Tagen) in Gruppe B mit 7,2\%. Nach Matching war diese in Gruppe A mit 3,7 \% am größten und in Gruppe B mit 1,1\% am kleinsten. Das bessere Abschneiden von Gruppe B ist möglicherweise auch auf die Weiterentwicklung der Intensivtherapie mit Fortschreiten der Zeit zurückzuführen. Beide Ergebnisse waren jedoch nicht signifikant.

Der Überlebensstatus im letzten Follow-up zeigte in Gruppe $\mathrm{C}$ die meisten Überlebenden ( $81,7 \%$ ) und in Gruppe A die meisten verstorbenen Patienten (23,3\%). Ein signifikanter Unterschied zwischen den Gruppen bestand nicht. Nach Matching zeigte Gruppe B das signifikant beste Ergebnis mit 90,9\% Überlebenden; die signifikant meisten Patienten verstarben wiederum in Gruppe A mit 23,0 \%, was am ehesten aufgrund der längsten Follow-up-Zeit zu erklären ist.

Auch die Überlebensanalyse anhand des Kaplan-Meier-Schätzers erbrachte initial ein signifikant geringeres Überleben der Gruppe B mit 78 \% nach 
5 Jahren und $65 \%$ nach 10 Jahren, im Vergleich zu den Gruppen A und C mit ca. $90 \%$ nach 5 Jahren und $80 \%$ nach 10 Jahren.

Nach Matching gab es zwischen den drei Gruppen beim Überleben keinen signifikanten Unterschied mehr. Hier zeigt sich deutlich, dass das initial schlechtere Outcome von Gruppe B wohl maßgeblich auf präoperative Risikofaktoren und zusätzliche Klappeneingriffe (v. a. TKR) zurückzuführen war, die in Gruppe B mit 49,5\% am häufigsten durchgeführt wurden.

In der Überlebensanalyse des ursprünglichen Patientenkollektivs verglichen mit der Vergleichspopulation zeigte sich in allen drei Gruppen eine 5 bis $10 \%$ höhere Sterblichkeit als bei der Normalbevölkerung. Zwischen den Gruppen bestand kein signifikanter Unterschied, wenngleich in Gruppe B eine höhere Sterblichkeit und in Gruppe $C$ die geringste verzeichnet werden konnte. Das Ausmaß der Sterblichkeit wird entscheidend bestimmt durch den Anteil der Frühsterblichkeit. In der Nachbeobachtungszeit verlaufen die Kaplan-MeierKurven weitestgehend parallel.

In einer Studie von Turkyilmaz et al. (2018) an 135 Patienten wurde das Outcome von superiorer transseptaler Inzision und linksatrialer Inzision nach Propensity Matching verglichen. Die Hospitalisierungszeit und der Aufenthalt auf der Intensivstation waren in Gruppe B signifikant länger. Hinsichtlich der Mortalität zeigte sich jedoch kein signifikanter Unterschied. ${ }^{96}$ Auch in den o. g. Studien zur Evaluation der Inzidenzen von Vorhofflimmern oder Schrittmacherimplantationen wurde die Mortalität von (superior) transseptalem und linksatrialem Zugang untersucht. Hier zeigte sich in den Überlebensraten vielfach kein signifikanter Unterschied zwischen diesen beiden Gruppen. ${ }^{34,74,80-82}$ Untersuchungen zur transcavalen Inzision konnten auch hier nicht gefunden werden.

\subsection{Multivariate Analysen}

In den multivariaten Analysen, insbesondere nach Matching, konnten die bisher geschilderten Ergebnisse bestätigt werden. 
Untersucht wurden insbesondere der Einfluss der atrialen Inzision und Ablation auf die Schrittmacherhäufigkeit sowie der Einfluss der atrialen Inzision, des linksatrialen präoperativen Durchmessers und des Alters auf den

Ablationserfolg.

Ein präoperativer pulmonaler Hypertonus hatte in beiden Betrachtungen keinen signifikanten Einfluss.

\subsection{Untersuchung verschiedener Merkmale auf die Schrittmacherhäufigkeit}

In der multivariaten Analyse mittels Cox-Regression nach Matching bestätigte sich, dass in Gruppe C das Risiko für Schrittmacherimplantationen im Vergleich zu Gruppe B signifikant erhöht ist ( $p=0.03$, HR 2.27).

Außerdem gehen im Gesamtkollektiv eine additive Ablation $(p<0.0001, H R$ 4.27) sowie hohes Alter ( $p=0,001, H R 1,05)$ mit einem höheren Risiko für Schrittmacherimplantationen einher.

Diese Ergebnisse waren vor und nach Matching im Wesentlichen gleich. Einzige Veränderung nach Matching war, dass Gruppe A das Schrittmacherrisiko nicht mehr signifikant gegenüber $B$ reduzierte, so dass es zwischen den beiden Gruppen keinen Unterschied mehr gab. Gruppe B war vermutlich vor Matching aufgrund anderer bereits genannter Faktoren mit einem höheren Risiko einhergegangen, die sich nach Matching anglichen.

Im Gegensatz zu den Ergebnissen von Utley et al. (1995) war die Art der atrialen Inzision in unserer Studie ein signifikanter Prädiktor für die permanente Schrittmacherimplantation. ${ }^{76}$

Auch Cho et al. (2018) zeigten mittels Cox-Regression, dass die additive MazeProzedur ) im Vergleich zur Mitralklappenchirurgie ohne Ablation ein höheres Risiko für das Sick-Sinus-Syndrom ( $p<0,001$, HR 7.44) und Schrittmacherimplantationen ( $p<0,001$, HR 3,61 mit sich bringt. Ein SickSinus-Syndrom entwickelten insgesamt 35 von 750 Patienten. 
Die Inzidenz war steigend, nach 1, 2 und 4 Jahren postoperativ mit 2,9\%, 3,7 \% bzw. 4,3\%. Die Patienten, die einen Schrittmacher bekamen, hatten außerdem höhere Raten von Schlaganfällen und Tod innerhalb der ersten vier Jahre. Auch in unserer Kaplan-Meier-Kurve bzgl. der

Schrittmacherimplantationen spiegelt sich die mit den Jahren steigende Inzidenz wieder. Nach 5 Jahren waren noch mehr Patienten frei von Schrittmachern als nach 10 Jahren.

Cho et al. differenzierten außerdem zwischen biatrialer und linksatrialer MazeProzedur. Das Fazit dieser Studie war, eine Minimierung der Läsionen im Sinne der linksatrialen Maze zu bevorzugen. Die biatriale Maze zeigte keinen Vorteil in der Effizienz bzgl. Vohofflimmerrezidiven, jedoch ein signifikant höheres Risiko für die Entwicklung eines Sick-Sinus-Syndroms. ${ }^{97}$

\subsection{Untersuchung verschiedener Merkmale auf den Ablationserfolg}

In der multivariaten Analyse mittels logistischer Regression nach Matching wurde der Herzrhythmus bei Entlassung und im letzten Follow-up nach Ablation betrachtet.

Für den kurzfristigen Ablationserfolg (postoperativ) war Gruppe A ein Prädiktor und verringerte das Risiko für Vorhofflimmern signifikant im Vergleich zu Gruppe B ( $p=0.039$, OR 0.15). Ebenfalls war ein hohes Alter mit einem erhöhten Risiko für Vorhofflimmern trotz Ablation assoziiert $(p=0.006$, OR 1.113). Der transcavale Zugang (Gruppe C) sowie die LA-Größe hatten hier keinen signifikanten Einfluss.

In der Langzeitbeobachtung (letztes Follow-up) war bei Gruppe $C$ das Risiko für Vorhofflimmern deutlich kleiner, der Ablationserfolg also signifikant größer als bei $B(p=0.01$, OR 0.09). Ursächlich war hierfür wahrscheinlich wieder die lange Follow-up-Zeit von Gruppe A und damit die zum Zeitpunkt des letzten Follow-ups bestehende höhere Anzahl an Vorhofflimmerrezidiven. Damit hatte Gruppe A im Vergleich zu Gruppe B keinen signifikanten Einfluss mehr auf den Ablationserfolg. 
Das Alter (in Jahren) blieb ein starker negativer Einfluss auf den Ablationserfolg $(p=0.001$, OR 1,14). In der Langzeitbeobachtung bestätigte sich - wie auch in anderen Studien -, dass ein vergrößerter linker Vorhof einen signifikant schlechten Einfluss auf den Ablationserfolg hat, also das Risiko für VHF deutlich erhöht $(p=0.02$, OR 3,18$)$.

Der Einfluss des linksatrialen Durchmessers, der Dauer des Vorhofflimmerns sowie des Alters wurde in Studien vielfach diskutiert.

Chen et al. (2012) untersuchten die additive Ablation (Maze IV) in der Klappenund Bypasschirurgie. In der logistischen Regression zeigte sich der präoperative linksatriale Durchmesser als unabhängiger Prädiktor für die Sinuskonversion nach Maze-Prozedur. ${ }^{70}$ Auch die Dauer des Vorhofflimmerns spielte hier (wie in anderen Studien) eine entscheidende Rolle. Je länger das Vorhofflimmern andauert, desto geringer die Sinuskonversionsraten. ${ }^{71}$ Der Zusammenhang zwischen dem Übergang von paroxysmalem zu permanentem Vorhofflimmern durch das fortschreitende strukturelle Remodeling mit linksatrialer Vergrößerung ist beschrieben. ${ }^{48,98}$

Stulak et al. (2007) analysierten das Outcome nach chirurgischer Ablation mit der Cox-Maze cut-and-sew-Methode an 335 Patienten, ebenfalls additiv zur Mitral-, Trikuspidal- und Bypasschirurgie. Nach $42 \pm 6$ SD Monaten waren $88 \%$ der gesamten Patienten im Sinusrhythmus, wobei die Vorhofflimmerfreiheit bei der Mitralklappenchirurgie geringer war als bei den anderen Eingriffen. In der multivariaten Analyse war die linksatriale Vergrößerung ein signifikanter Prädiktor für ein Vorhofflimmerrezidiv. Der linksatriale Durchmesser war bei Patienten ohne Rezidiv signifikant kleiner. ${ }^{99}$

Eine neue Erkenntnis unserer Studie ist, dass die Art der atrialen Inzision einen Einfluss auf den Ablationserfolg zu haben scheint. Diesbezüglich bedarf es weiterer Studien mit einheitlichen Follow-up-Zeiten, um beurteilen zu können, ob die linksatriale oder die transcavale Inzision den längerfristigen Ablationserfolg bringt. 


\subsection{Stärken und Schwächen der Studie}

Die Stärken unserer Studie liegen in der großen Patientenzahl, verbunden mit $92 \%$ Follow-up-Teilnahme und langer Follow-up-Zeit von $76 \pm$ 49,4 SD Monaten, sowie in der Einbeziehung des transcavalen Zugangs, der in der Literatur bis dato kaum beschrieben ist. Die statistischen Analysen waren umfangreich und wurden mittels erneuter Auswertung nach erfolgtem Propensity Matching noch aussagekräftiger.

Die zunächst vorgenommene Auswertung mit dem originalen Patientenstamm beinhaltete signifikante Unterschiede in der Patientenanzahl pro Gruppe und in wesentlichen, die Fragestellung beeinflussenden Faktoren wie Zusatzeingriffe, Alter etc. Diese Limitation konnte mittels Propensity Matching in den wesentlichen Punkten beseitigt werden.

Weitere problematische Punkte waren die sehr unterschiedliche Follow-upDauer und die Tatsache, dass die postoperativ erhobenen Follow-ups zu sehr verschiedenen Zeitpunkten stattfanden.

In den frühen Jahren des in die Studie eingeschlossenen Zeitraums waren Patienten vor allem über den der Gruppe A zugeteilten Zugang operiert worden. Gleichzeitig wurden damals noch nicht so konsequent Follow-ups generiert. Das bedeutet: Wenn ein Patient der Gruppe A in einem Follow-up 10 Jahre postoperativ Vorhofflimmern angegeben hatte, konnte nicht eruiert werden, seit wann dies bestand. Daher konnten die Fragen „Wann bekommen die Patienten postoperativ VHF?“" oder „Wie lange hält der SR nach Ablation?“ nicht beantwortet werden.

Wenn feste Daten wie das Implantationsdatum eines Herzschrittmachers oder das Todesdatum angegeben wurden, konnten mittels Kaplan-Meier-Schätzer Aussagen über den Verlauf nach bspw. 5 und 10 Jahren getroffen werden. Feste Follow-up-Zeitpunkte für alle Patienten der Studie hätten die Auswertung vereinfacht und hätten es ermöglicht, die o. g. Fragestellungen zu beantworten. 
Allerdings bleibt der Bias des evtl. unentdeckten paroxysmalen Vorhofflimmerns in diesem Kontext immer bestehen.

Grundsätzlich bestanden die Nachuntersuchungen aus einem Fragebogen, der von den Patienten selbst beantwortet wurde, sowie ergänzenden Befunden der THG-Ambulanz oder niedergelassener Kardiologen. Natürlich unterliegen diese Unterlagen einem starken subjektiven Faktor.

\subsection{Schlussfolgerungen und Ausblick}

Die beiden Kernthemen dieser Studie, Vorhofflimmern und Mitralklappenvitien, sind aktuell und bleiben auch in Zukunft von großer Relevanz.

Die Ergebnisse der vorliegenden Studie zeigen, dass der Zugangsweg zur Mitralklappe für postoperatives Vorhofflimmern, den Ablationserfolg und die Schrittmacherinzidenz eine entscheidende Rolle spielt.

In unserer Studie konnte im Hinblick auf das postoperativ neu aufgetretene Vorhofflimmern zwar kein signifikanter Unterschied zwischen den drei Gruppen gezeigt werden, bei Gruppe B kam es jedoch - wie auch in anderen Studien mit statistischer Signifikanz gezeigt - häufiger vor. ${ }^{74-76}$

Auch im Hinblick auf die Schrittmacherinzidenz wurde gezeigt, dass insbesondere die superiore transseptale Inzision (wie in unserer Studie angewandt) postoperative Rhythmusstörungen und Schrittmacherimplantationen aufgrund einer Sinusknotendysfunktion begünstigen kann. ${ }^{33,74,78,79,81,82}$

Auch wenn die Studienlage bzgl. der zugrunde liegenden Verletzung der Sinusknotenarterie nicht eindeutig ist, gibt es bereits Alternativen und modifizierte Techniken, die es weiterzuentwickeln gilt. ${ }^{83,84}$

Dass Gruppe C sowohl ohne als auch mit additiver Ablation das signifikant höchste Schrittmacherrisiko hatte, kann bis dato nicht erklärt werden. 
Die atriale Inzision entspricht im Wesentlichen der von Gruppe A, die die geringste Zahl an Schrittmacherimplantationen hatte. Hier bedarf es weiterer Untersuchungen des transcavalen Zugangs, um Erklärungen zu finden. In der multivariaten Analyse konnte gezeigt werden, dass Gruppe $C$ das Risiko für Schrittmacherimplantationen im Vergleich zu Gruppe B signifikant erhöht und die atriale Inzision damit einen entscheidenden Einfluss auf die Schrittmacherinzidenz hat. Die additive Ablation sowie hohes Alter waren ebenfalls starke Prädiktoren für ein erhöhtes Schrittmacherrisiko.

Die Effizienz der additiven Ablation wie auch das dadurch erhöhte Schrittmacherrisiko konnten in unserer Studie bestätigt werden. ${ }^{72,73,87-89}$ Zusammenfassend zeigte in unserer Arbeit Gruppe A die postoperativ signifikant beste Konversionsrate, jedoch verbunden mit höchster Rezidivrate bei längster Follow-up-Zeit). Im Vergleich zu anderen Studien war dies ein sehr gutes Ergebnis. ${ }^{93,100}$

Gemäß der Langzeitbeobachtung waren in Gruppe C die meisten Patienten mit anhaltendem Sinusrhythmus. Die geringeren Ablationserfolge von Gruppe B können möglicherweise auf den Beginn der Durchführung dieser Technik (mit zunehmender Verbesserung in Folge spezifischer Publikationen in den späteren Jahren) zurückzuführen sein. ${ }^{88,92}$ In der multivariaten Analyse war Gruppe A ein Prädiktor für den kurzfristigen Ablationserfolg (postoperativ) und verringerte das Risiko für Vorhofflimmern signifikant im Vergleich zu Gruppe B. Höheres Alter war ein signifikant negativer Einfluss auf den Ablationserfolg.

In der Langzeitbeobachtung (letztes Follow-up) war bei Gruppe C das Risiko für Vorhofflimmern deutlich kleiner, der Ablationserfolg also signifikant größer als bei B. Gruppe A hatte hier - möglicherweise aufgrund der wegen des späten Zeitpunktes des Follow-ups häufigeren Vorhofflimmerrezidive - keinen signifikanten Einfluss mehr. Das Alter blieb ein starker negativer Einfluss auf den Ablationserfolg. 
Auch eine linksatriale Vergrößerung war - wie in anderen Studien vielfach beschrieben - nach unseren Ergebnissen ein signifikanter negativer Prädiktor für den Ablationserfolg. ${ }^{70,99}$ Scherer et al. ${ }^{101}$ konnten jedoch zeigen, dass durch chirurgische Vorhofverkleinerung dieser Risikofaktor zumindest verkleinert werden kann.

Die Mortalität der drei Zugangswege ist laut unserer Studie vergleichbar. Nach Egalisierung der Störfaktoren durch Matching gab es diesbezüglich keinen signifikanten Unterschied zwischen den Gruppen.

Es bedarf weiterer Studien, um den in unseren Ergebnissen aufgezeigten Einfluss der atrialen Inzision als Zugang zur Mitralklappe auf den Herzrhythmus, die Schrittmacherinzidenz und den Ablationserfolg beurteilen zu können. Insbesondere der transcavale Zugang, der sich im negativen Sinne auf die Zahl der Schrittmacherimplantationen auszuwirken scheint, gibt Anlass für weitere Untersuchungen. Diese Studien sollten prospektiv erfolgen und einheitliche Follow-up-Zeitpunkte beinhalten, um eine optimale Vergleichbarkeit herzustellen.

Diese Erkenntnisse könnten einen wichtigen Beitrag leisten, um die Operationsmethoden in der Mitralklappenchirurgie zugunsten eines bestmöglichen Ablationserfolges und noch geringerer Schrittmacherinzidenzen zu optimieren. 


\section{Zusammenfassung}

Die vorliegende Studie befasst sich mit dem Einfluss minimalinvasiver Zugangswege zur Mitralklappe auf den Herzrhythmus, den Erfolg einer perioperativ durchgeführten Ablation und die postoperative Notwendigkeit eines Herzschrittmachers.

Mitralklappenvitien und deren herzchirurgische Versorgung sind in vielen Fällen mit präoperativ bestehendem oder postoperativ neu auftretendem Vorhofflimmern assoziiert. ${ }^{45}$ In den vergangenen Jahrzehnten haben sich neben der medikamentösen Therapie des Vorhofflimmerns und der durch die Mitralklappeninsuffizienz induzierten Herzinsuffizienz verschiedene minimalinvasive chirurgische Zugangswege zur Mitralklappe sowie Ablationsverfahren etabliert und einen kurativen Therapieansatz gebildet. Die Ablation im Zuge einer Mitralklappenchirurgie ist zu einem alltäglich durchgeführten Verfahren geworden.

Neu auftretendes Vorhofflimmern im Rahmen der Mitralklappenchirurgie kann perioperativ begrenzt sein und konvertiert häufig innerhalb der ersten 6 Wochen spontan in den Sinusrhythmus. Es geht aber mit einer erhöhten Mortalität und Hospitalisierungszeit einher. ${ }^{44}$ Das neu auftretende Vorhofflimmern kann jedoch auch persistieren oder erst im Langzeitverlauf entstehen. Auch die Notwendigkeit eines Herzschrittmachers kann durch Mitralklappeneingriffe insbesondere mit additiver Ablation aufgrund der anatomischen Gegebenheiten

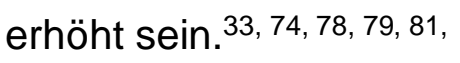

In unserer Arbeit ist von Interesse, ob sich die unten genannten Zugangswege im Hinblick auf das Neuauftreten von Vorhofflimmern im Langzeitverlauf, die Vorhofflimmerrezidivrate nach Ablation und die Schrittmacherrate mit und ohne durchgeführte Ablation unterscheiden.

Die vorliegende Studie umfasst alle Mitralklappenoperationen, die zwischen 1998 und 2015 in der Klinik für Thorax-, Herz- und thorakale Gefäßchirurgie der Universitätsklinik Frankfurt am Main über die folgenden drei minimalinvasiven Zugangswege, durchgeführt wurden: Gruppe A bildeten 300 Patienten, die im 
genannten Zeitraum über eine anterolaterale Minithorakotomie mittels Chitwood-Klemme operiert wurden. Gruppe B bestand aus 687 Patienten, die über eine partielle obere Sternotomie mit superiorem transseptalem Zugang operiert wurden. Die 219 Patienten, bei denen eine partielle obere Sternotomie mit transcavalem Zugang angewandt wurde, bildeten Gruppe C.

Die Auswertung erfolgte anhand von Patientenakten, internen und externen Untersuchungsbefunden und eines standardisierten Fragebogens im Follow-up. Es erfolgte eine zweite Auswertung nach Propensity Matching, um präoperative signifikante Unterschiede der Gruppen zu egalisieren.

Im Ergebnis konnte in unserer Studie gezeigt werden, dass die atriale Inzision einen entscheidenden Einfluss auf den Ablationserfolg sowie auf die Schrittmacherinzidenz hat. Bekannt war dabei ein höheres Risiko für postoperatives Vorhofflimmern und Schrittmacherimplantationen aufgrund der anatomischen Gegebenheiten bei Gruppe B. Dass jedoch Gruppe $C$ ein signifikant noch höheres Risiko für Schrittmacherimplantationen mit sich bringt, war überraschend und ist derzeit nicht in der Literatur beschrieben. In der multivariaten Analyse nach Matching waren Gruppe C, eine additive Ablation und das Alter signifikante unabhängige Prädiktoren für Schrittmacherimplantationen. In der logistischen Regression war Gruppe A ein unabhängiger Prädiktor für den Ablationserfolg zum Zeitpunkt der Entlassung. In der Langzeitbeobachtung trat Gruppe $\mathrm{C}$ an Stelle von Gruppe A, möglicherweise aufgrund der bei Gruppe A vorliegenden längsten Follow-upZeit. Wie schon in der vorliegenden Literatur diskutiert waren auch in unserer Auswertung hohes Alter und eine präoperative linksatriale Vergrößerung unabhängige Prädiktoren für den langfristigen Ablationserfolg. ${ }^{70,99}$

Es bedarf weiterer vergleichender Studien mit einheitlichen Follow-up-Zeiten, um die hier gezeigten Ergebnisse zu überprüfen. Insbesondere der transcavale Zugang, der sich im negativen Sinne auf die Zahl der Schrittmacherimplantationen auszuwirken scheint, gibt Anlass für weitere Untersuchungen. 


\section{Summary}

This study focuses on the influence of minimally invasive access routes to the mitral valve on cardiac rhythm, the success of ablation performed perioperatively, and the postoperative need for a cardiac pacemaker. Mitral valve defects and their treatment by means of cardiac surgery are associated in many cases with atrial fibrillation which exists preoperatively or which newly develops postoperatively. ${ }^{45}$ In the past few decades, various minimally invasive surgical access routes to the mitral valve as well as ablation methods have been established alongside medical therapy of atrial fibrillation and of heart failure induced by the mitral valve insufficiency, and these have formed a curative therapeutic approach. The ablation in the course of mitral valve surgery has become a commonplace procedure.

Atrial fibrillation which newly occurs within the setting of mitral valve surgery can be perioperatively limited and frequently converts spontaneously to sinus rhythm within the first 6 weeks. However, it is associated with increased mortality and hospitalization time. ${ }^{44}$ The newly occurring atrial fibrillation may also persist, however, or only develop during the long-term course.

The need for a cardiac pacemaker may also be increased through mitral valve procedures, particularly with additive ablation, due to the anatomical

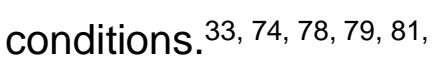

Our work focuses on whether the routes of access listed below differ with regard to the new appearance of atrial fibrillation over the long term, the rate of recurrence of atrial fibrillation after ablation, and the pacemaker rate with and without performing ablation.

This study includes all mitral valve surgeries which were performed between 1998 and 2015 in the Department of Thoracic and Cardiovascular Surgery of the Frankfurt am Main University Hospital using the following three minimally invasive access routes: Group A included 300 patients who underwent surgery in the time period indicated via an anterolateral mini thoracotomy using a Chitwood clamp. Group B comprised 687 patients who underwent surgery by 
means of a partial upper sternotomy with superior transseptal access. The 219 patients in whom a partial upper sternotomy with transcaval access was used comprised group $\mathrm{C}$. The evaluation was performed using patient medical records, internal and external examination findings, and a standardized questionnaire in the follow-up. A second evaluation according to propensity matching was performed in order to equalize significant preoperative differences between the groups.

In the result, it was able to be shown in our study that the atrial incision has a decisive influence on the success of the ablation as well as on the pacemaker incidence. A higher risk of postoperative atrial fibrillation and pacemaker implantation due to the anatomical conditions was known in group B. However, the fact that group $\mathrm{C}$ entails a significantly even higher risk of pacemaker implantations was surprising and is currently not described in the literature. In the multivariate analysis after matching, group $\mathrm{C}$, an additive ablation, and age were significant independent predictors for pacemaker implantations. In the logistic regression, group A was an independent predictor for the success of the ablation at the time of discharge. In the long-term observation, group $\mathrm{C}$ took the place of group A, possibly due to the longest follow-up time which was present in the case of group A. As already discussed in the present literature, an advanced age and preoperative left atrial enlargement were independent predictors for the long-term success of the ablation in our evaluation as well. ${ }^{70,99}$

There is a need for additional comparative studies with uniform follow-up times in order to verify the results shown here. In particular, the transcaval approach, which appears to have an effect in the negative sense on the number of pacemaker implantations, gives a reason for further investigations. 


\section{Anhang}

\subsection{Fragebogen}

Fragebogen zur Erfassung des Gesundheitszustandes von Patienten nach einer Herzklappenoperation
Name
Datum

- Haben Sie belastungsabhängige Atemnot?

Nein

Wenn ja:

Bei starker körperlicher Belastung

Beim Treppensteigen (2.Stock) und beim Bergaufgehen

Beim langsamen gehen in der Ebene oder in Ruhe

- Nehmen Sie blutverdünnende Medikamente ein?

Nein

Ja

Wenn ja welche?

ASS (Aspirin)

Plavix (Clopidogrel)

Marcumar

andere:

Warum?

Vorhofflimmern

Venenthrombose

andere

- Haben Sie Rhythmusstörungen?

Nein

Vorhofflimmern

andere:

Schrittmachertherapie seit 
- Kam es seit der Herzklappenoperation zu einer Komplikation?

a.) Embolie

Nein

$\mathrm{Ja}$

Wenn ja welcher Art:

Gehirn (Schlaganfall, vorübergehende Sehschwäche oder

Sprachstörung)

Beine

Herz (Herzinfarkt)

andere

Welche Symptome haben Sie dabei gehabt

Wann und wo wurde diese behandelt

Haben Sie noch Restbeschwerden?

$\mathrm{Ja}$

Nein

b.) Klappenendokarditis

Nein

$\mathrm{Ja}$

Wenn ja wann

Wo wurde diese behandelt

Welche Beschwerden hatten Sie

\section{c.) Störung der Klappenfunktion}

Nein

$\mathrm{Ja}$

Wenn ja welcher Art:

Undichtigkeit

Stenose (Klappenverengung) 
Sind sie deswegen operiert worden?

Nein

Ja

Wenn ja wann wo

d.) Blutung

Nein

$\mathrm{Ja}$

Wenn ja welcher Art:

Hautblutung

Magenblutung

Harnwegsbutung

Hirnblutung

andere

Waren sie deswegen im Krankenhaus aufgenommen?

Nein

$\mathrm{Ja}$

Wenn ja wann wo

Behandlung

Haben sie deswegen Blutkonserven erhalten?

Nein

$\mathrm{Ja}$

Wie hoch war ihr Quick-Wert oder INR zum Zeitpunkt der Blutung?

Quick $\% \quad$ INR

- Sind in der Zwischenzeit andere, schwere Erkrankungen aufgetreten?

Nein

$\mathrm{Ja}$ 
- Sind sie bereit an einer Echokardiografie (Herzultraschall) teilzunehmen?

Nein

$\mathrm{Ja}$

Wenn ja, wann

Bitte, wenn möglich, eine Kopie des letzten Befundes mitschicken.

Wir bitten Sie um Erlaubnis, Ihre Daten zur Qualitätskontrolle bei uns zu

speichern und uns gegebenenfalls bei Ihren behandelnden Ärzten über herzklappenbezogene Probleme zu erkundigen.

$\square$ Einverstanden

Patientenunterschrift, Telefon

Kardiologe o. Hausarzt, Telefon 


\subsection{Schriftliche Erklärung}

Ich erkläre ehrenwörtlich, dass ich die dem Fachbereich Medizin der Johann Wolfgang Goethe-Universität Frankfurt am Main zur Promotionsprüfung eingereichte Dissertation mit dem Titel

Veränderungen des Herzrhythmus nach minimalinvasiver Mitralklappenchirurgie

Am Zentrum für Chirurgie, Klinik für Thorax-, Herz- und thorakale Gefäßchirurgie unter Betreuung und Anleitung von Prof. Dr. Anton Moritz mit Unterstützung durch Dipl. Ing. Afsaneh Karimian-Tabrizi ohne sonstige Hilfe selbst durchgeführt und bei der Abfassung der Arbeit keine anderen als die in der Dissertation angeführten Hilfsmittel benutzt habe. Darüber hinaus versichere ich, nicht die Hilfe einer kommerziellen Promotionsvermittlung in Anspruch genommen zu haben.

Ich habe bisher an keiner in- oder ausländischen Universität ein Gesuch um Zulassung zur Promotion eingereicht. ${ }^{*}$ Die vorliegende Arbeit wurde bisher nicht als Dissertation eingereicht.

Berlin, 17.07.2020

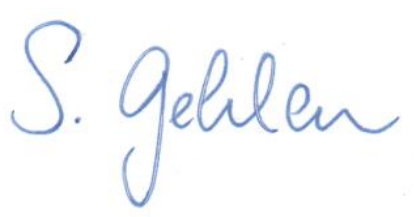

(Ort, Datum)

(Unterschrift)

*) im Falle des Nichtzutreffens entfernen 


\section{Literaturverzeichnis}

1. Fenster MS, Feldman MD. Mitral regurgitation: an overview. Current problems in cardiology. 1995;20(4):193-280.

2. lung B, Baron $\mathrm{G}$, Butchart $\mathrm{EG}$, et al. A prospective survey of patients with valvular heart disease in Europe: The Euro Heart Survey on Valvular Heart Disease. European heart journal. 2003;24(13):1231-1243.

3. Klein AL, Burstow DJ, Tajik AJ, et al. Age-related prevalence of valvular regurgitation in normal subjects: a comprehensive color flow examination of 118 volunteers. Journal of the American Society of Echocardiography : official publication of the American Society of Echocardiography. 1990;3(1):54-63.

4. Herold G ea. Innere Medizin. 2014.

5. Athanasuleas CL, Stanley AWH, Buckberg GD. Mitral regurgitation: anatomy is destiny. Eur J Cardiothorac Surg. 2018.

6. Reichek N, Shelburne JC, Perloff JK. Clinical aspects of rheumatic valvular disease. Progress in cardiovascular diseases. 1973;15(5):491537.

7. Nickenig G, Mohr FW, Kelm M, et al. Konsensus der Deutschen Gesellschaft für Kardiologie - Herz- und Kreislaufforschung - und der Deutschen Gesellschaft für Thorax-, Herz- und Gefäßchirurgie zur Behandlung der Mitralklappeninsuffizienz. Der Kardiologe. 2013;7(2):7690.

8. Enriquez-Sarano M, Akins CW, Vahanian A. Mitral regurgitation. Lancet (London, England). 2009;373(9672):1382-1394.

9. Otto CM. Timing of surgery in mitral regurgitation. Heart. 2003;89(1):100105.

10. Cohn LH. Surgery for mitral regurgitation. Jama. 1988;260(19):28832887.

11. Acker MA, Parides MK, Perrault LP, et al. Mitral-valve repair versus replacement for severe ischemic mitral regurgitation. The New England journal of medicine. 2014;370(1):23-32.

12. Boekstegers $P$, Hausleiter J, Baldus S, et al. Interventionelle Behandlung der Mitralklappeninsuffizienz mit dem MitraClip®-Verfahren. Der Kardiologe. 2013;7(2):91-104.

13. Enriquez-Sarano M, Sundt TM, 3rd. Early surgery is recommended for mitral regurgitation. Circulation. 2010;121(6):804-811; discussion 812.

14. Falk V, Baumgartner H, Bax JJ, et al. 2017 ESC/EACTS Guidelines for the management of valvular heart disease. Eur J Cardiothorac Surg. 2017;52(4):616-664.

15. Enriquez-Sarano M, Avierinos JF, Messika-Zeitoun D, et al. Quantitative determinants of the outcome of asymptomatic mitral regurgitation. The New England journal of medicine. 2005;352(9):875-883. 
16. Cleland JGF, Daubert J-C, Erdmann E, et al. The Effect of Cardiac Resynchronization on Morbidity and Mortality in Heart Failure. New England Journal of Medicine. 2005;352(15):1539-1549.

17. Deja MA, Grayburn PA, Sun B, et al. Influence of mitral regurgitation repair on survival in the surgical treatment for ischemic heart failure trial. Circulation. 2012;125(21):2639-2648.

18. Wunderlich NC, Dalvi B, Ho SY, Kux H, Siegel RJ. Rheumatic Mitral Valve Stenosis: Diagnosis and Treatment Options. Current cardiology reports. 2019;21(3):14.

19. Selzer A, Cohn KE. Natural history of mitral stenosis: a review. Circulation. 1972;45(4):878-890.

20. Waller BF, Howard J, Fess S. Pathology of mitral valve stenosis and pure mitral regurgitation--Part I. Clinical cardiology. 1994;17(6):330-336.

21. Bryan AJ, Lamarra M, Angelini GD, West RR, Breckenridge IM. Median sternotomy wound dehiscence: a retrospective case control study of risk factors and outcome. Journal of the Royal College of Surgeons of Edinburgh. 1992;37(5):305-308.

22. Zacharias A, Habib $\mathrm{RH}$. Factors predisposing to median sternotomy complications. Deep vs superficial infection. Chest. 1996;110(5):11731178.

23. Goodman LR, Kay HR, Teplick SK, Mundth ED. Complications of median sternotomy: computed tomographic evaluation. AJR American journal of roentgenology. 1983;141(2):225-230.

24. Carpentier A, Loulmet D, Carpentier A, et al. [Open heart operation under videosurgery and minithoracotomy. First case (mitral valvuloplasty) operated with success]. Comptes rendus de l'Academie des sciences Serie III, Sciences de la vie. 1996;319(3):219-223.

25. Cosgrove DM, 3rd, Sabik JF, Navia JL. Minimally invasive valve operations. Ann Thorac Surg. 1998;65(6):1535-1538; discussion 15381539.

26. Cohn LH, Adams DH, Couper GS, et al. Minimally invasive cardiac valve surgery improves patient satisfaction while reducing costs of cardiac valve replacement and repair. Annals of surgery. 1997;226(4):421-426; discussion 427-428.

27. Doenst T, Lamelas J. Do we have enough evidence for minimallyinvasive cardiac surgery? A critical review of scientific and non-scientific information. The Journal of cardiovascular surgery. 2017;58(4):613-623.

28. Chitwood WR, Jr., Elbeery JR, Chapman WH, et al. Video-assisted minimally invasive mitral valve surgery: the "micro-mitral" operation. $J$ Thorac Cardiovasc Surg. 1997;113(2):413-414.

29. Martens S, Dietrich M, Doss M, Wimmer-Greinecker G, Moritz A. Optimal carbon dioxide application for organ protection in cardiac surgery. $J$ Thorac Cardiovasc Surg. 2002;124(2):387-391. 
30. Reser D, Holubec T, Yilmaz M, Guidotti A, Maisano F. Right lateral minithoracotomy for mitral valve surgery. Multimedia manual of cardiothoracic surgery : MMCTS. 2015;2015.

31. Svensson LG. Minimal-access "J" or "j" sternotomy for valvular, aortic, and coronary operations or reoperations. Ann Thorac Surg. 1997;64(5):1501-1503.

32. Navia JL, Cosgrove DM, 3rd. Minimally invasive mitral valve operations. The Annals of thoracic surgery. 1996;62(5):1542-1544.

33. Lukac P, Hjortdal VE, Pedersen AK, Mortensen PT, Jensen HK, Hansen PS. Superior Transseptal Approach to Mitral Valve Is Associated With a Higher Need for Pacemaker Implantation Than the Left Atrial Approach. The Annals of Thoracic Surgery. 2007;83(1):77-82.

34. Masiello P, Triumbari F, Leone R, Itri F, Del Negro G, Di Benedetto G. Extended vertical transseptal approach versus conventional left atriotomy for mitral valve surgery. The Journal of heart valve disease. 1999;8(4):440-444.

35. Barner HB. Combined superior and right lateral left atriotomy with division of the superior vena cava for exposure of the mitral valve. Ann Thorac Surg. 1985;40(4):365-367.

36. Zoni-Berisso M, Lercari F, Carazza T, Domenicucci S. Epidemiology of atrial fibrillation: European perspective. Clinical epidemiology. 2014;6:213-220.

37. Chugh SS, Havmoeller R, Narayanan K, et al. Worldwide epidemiology of atrial fibrillation: a Global Burden of Disease 2010 Study. Circulation. 2014;129(8):837-847.

38. Miyasaka Y, Barnes ME, Gersh BJ, et al. Secular trends in incidence of atrial fibrillation in Olmsted County, Minnesota, 1980 to 2000, and implications on the projections for future prevalence. Circulation. 2006;114(2):119-125.

39. Ball J, Carrington MJ, McMurray JJ, Stewart S. Atrial fibrillation: profile and burden of an evolving epidemic in the 21st century. International journal of cardiology. 2013;167(5):1807-1824.

40. Benjamin EJ, Levy D, Vaziri SM, D'Agostino RB, Belanger AJ, Wolf PA. Independent risk factors for atrial fibrillation in a population-based cohort. The Framingham Heart Study. Jama. 1994;271(11):840-844.

41. Benjamin EJ, Wolf PA, D'Agostino RB, Silbershatz $H$, Kannel WB, Levy D. Impact of atrial fibrillation on the risk of death: the Framingham Heart Study. Circulation. 1998;98(10):946-952.

42. Stewart S, Hart CL, Hole DJ, McMurray JJ. A population-based study of the long-term risks associated with atrial fibrillation: 20-year follow-up of the Renfrew/Paisley study. The American journal of medicine. 2002;113(5):359-364.

43. Fox CS, Parise H, D'Agostino RB, Sr., et al. Parental atrial fibrillation as a risk factor for atrial fibrillation in offspring. Jama. 2004;291(23):28512855. 
44. Maisel WH, Rawn JD, Stevenson WG. Atrial fibrillation after cardiac surgery. Annals of internal medicine. 2001;135(12):1061-1073.

45. Auer J, Weber T, Berent R, Ng C-K, Lamm G, Eber B. Risk Factors of Postoperative Atrial Fibrillation After Cardiac Surgery. Journal of Cardiac Surgery. 2005;20(5):425-431.

46. Haissaguerre $M$, Jais $P$, Shah DC, et al. Spontaneous initiation of atrial fibrillation by ectopic beats originating in the pulmonary veins. The New England journal of medicine. 1998;339(10):659-666.

47. Cox JL, Boineau JP, Schuessler RB, Jaquiss RD, Lappas DG.

Modification of the maze procedure for atrial flutter and atrial fibrillation. I. Rationale and surgical results. J Thorac Cardiovasc Surg. 1995;110(2):473-484.

48. $\quad$ Nguyen BL, Fishbein MC, Chen LS, Chen PS, Masroor S. Histopathological substrate for chronic atrial fibrillation in humans. Heart rhythm. 2009;6(4):454-460.

49. Knapp E, Watson K. Medication management of atrial fibrillation: emerging therapies for rhythm control and stroke prevention. $P \& T: a$ peer-reviewed journal for formulary management. 2011;36(8):518-528.

50. Lim HS, Willoughby SR, Schultz C, et al. Effect of atrial fibrillation on atrial thrombogenesis in humans: impact of rate and rhythm. J Am Coll Cardiol. 2013;61(8):852-860.

51. Hart RG, Pearce LA, Aguilar MI. Meta-analysis: antithrombotic therapy to prevent stroke in patients who have nonvalvular atrial fibrillation. Annals of internal medicine. 2007;146(12):857-867.

52. Reiffel JA. Atrial fibrillation and stroke: epidemiology. The American journal of medicine. 2014;127(4):e15-16.

53. Holmes DR, Reddy VY, Turi ZG, et al. Percutaneous closure of the left atrial appendage versus warfarin therapy for prevention of stroke in patients with atrial fibrillation: a randomised non-inferiority trial. Lancet (London, England). 2009;374(9689):534-542.

54. Hucker WJ, Cohen JA, Gurol ME, et al. WATCHMAN implantation in patients with a history of atrial fibrillation and intracranial hemorrhage. Journal of interventional cardiac electrophysiology : an international journal of arrhythmias and pacing. 2019.

55. Kleczynski P, Sorysz D, Zawislak B, et al. [Percutaneous left atrial appendage closure with Watchman(R) LAA occluder device in a patient with persistent atrial fibrillation]. Kardiologia polska. 2012;70(9):965-967.

56. Maan A, Mansour M, N Ruskin J, Heist EK. Current Evidence and Recommendations for Rate Control in Atrial Fibrillation. Arrhythm Electrophysiol Rev. 2013;2(1):30-35.

57. Al-Khatib SM, Allen LaPointe NM, Chatterjee R, et al. Rate- and rhythmcontrol therapies in patients with atrial fibrillation: a systematic review. Annals of internal medicine. 2014;160(11):760-773. 
58. Roy D, Talajic M, Nattel S, et al. Rhythm control versus rate control for atrial fibrillation and heart failure. The New England journal of medicine. 2008;358(25):2667-2677.

59. Yousef N, Philips M, Shetty I, Cui VW, Zimmerman F, Roberson DA. Transesophageal echocardiography of intracardiac thrombus in congenital heart disease and atrial flutter: the importance of thorough examination of the Fontan. Pediatric cardiology. 2014;35(7):1099-1107.

60. Boriani G, Diemberger I, Biffi M, et al. Electrical cardioversion for persistent atrial fibrillation or atrial flutter in clinical practice: predictors of long-term outcome. International journal of clinical practice. 2007;61(5):748-756.

61. Singh SN, Tang XC, Reda D, Singh BN. Systematic electrocardioversion for atrial fibrillation and role of antiarrhythmic drugs: a substudy of the SAFE-T trial. Heart rhythm. 2009;6(2):152-155.

62. Coplen SE, Antman EM, Berlin JA, Hewitt P, Chalmers TC. Efficacy and safety of quinidine therapy for maintenance of sinus rhythm after cardioversion. A meta-analysis of randomized control trials. Circulation. 1990;82(4):1106-1116.

63. Shah DC, Haissaguerre $M$, Jais $P$. Catheter ablation of pulmonary vein foci for atrial fibrillation: PV foci ablation for atrial fibrillation. The Thoracic and cardiovascular surgeon. 1999;47 Suppl 3:352-356.

64. Miyazaki S, Kuwahara T, Kobori A, et al. Long-term clinical outcome of extensive pulmonary vein isolation-based catheter ablation therapy in patients with paroxysmal and persistent atrial fibrillation. Heart. 2011;97(8):668-673.

65. Bradley DJ, Shen WK. Overview of management of atrial fibrillation in symptomatic elderly patients: pharmacologic therapy versus AV node ablation. Clinical pharmacology and therapeutics. 2007;81(2):284-287.

66. Cox JL, Boineau JP, Schuessler RB, Kater KM, Lappas DG. Five-year experience with the maze procedure for atrial fibrillation. Ann Thorac Surg. 1993;56(4):814-823; discussion 823-814.

67. Voeller RK, Bailey MS, Zierer A, et al. Isolating the entire posterior left atrium improves surgical outcomes after the Cox maze procedure. The Journal of Thoracic and Cardiovascular Surgery. 2008;135(4):870-877.

68. Gillinov AM. Advances in surgical treatment of atrial fibrillation. Stroke. 2007;38(2 Suppl):618-623.

69. Weimar T, Schena S, Bailey MS, et al. The cox-maze procedure for lone atrial fibrillation: a single-center experience over 2 decades. Circulation Arrhythmia and electrophysiology. 2012;5(1):8-14.

70. Chen MC, Chang JP, Chang HW. Preoperative atrial size predicts the success of radiofrequency maze procedure for permanent atrial fibrillation in patients undergoing concomitant valvular surgery. Chest. 2004;125(6):2129-2134. 
71. Geidel S, Krause K, Boczor S, et al. Ablation surgery in patients with persistent atrial fibrillation: an 8-year clinical experience. J Thorac Cardiovasc Surg. 2011;141(2):377-382.

72. Gillinov AM, Gelijns AC, Parides MK, et al. Surgical ablation of atrial fibrillation during mitral-valve surgery. The New England journal of medicine. 2015;372(15):1399-1409.

73. Bando K, Kobayashi J, Kosakai Y, et al. Impact of Cox maze procedure on outcome in patients with atrial fibrillation and mitral valve disease. The Journal of Thoracic and Cardiovascular Surgery. 2002;124(3):575-583.

74. Rezahosseini O, Rezaei M, Ahmadi Tafti SH, et al. Transseptal Approach versus Left Atrial Approach to Mitral Valve: A Propensity Score Matching Study. The journal of Tehran Heart Center. 2015;10(4):188193.

75. Lukac P, Hjortdal VE, Pedersen AK, Mortensen PT, Jensen HK, Hansen PS. Atrial incision affects the incidence of atrial tachycardia after mitral valve surgery. Ann Thorac Surg. 2006;81(2):509-513.

76. Utley JR, Leyland SA, Nguyenduy T. Comparison of outcomes with three atrial incisions for mitral valve operations: Right lateral, superior septal, and transseptal. The Journal of Thoracic and Cardiovascular Surgery. 1995;109(3):582-587.

77. Gialdini G, Nearing K, Bhave PD, et al. Perioperative atrial fibrillation and the long-term risk of ischemic stroke. JAMA. 2014;312(6):616-622.

78. Berdajs D, Patonay L, Turina MI. The clinical anatomy of the sinus node artery. Ann Thorac Surg. 2003;76(3):732-735; discussion 735-736.

79. Boulemden A, Nadarajah D, Szafranek AA, Richens D. Permanent pacemaker insertion postmitral surgery: Do the atrial access and the origin of the sinoatrial node artery matter? Journal of cardiac surgery. 2019;34(7):563-569.

80. Arslan A, Aydin E, Ozkokeli M. Comparison of Superior Septal Approach with Left Atriotomy in Mitral Valve Surgery. Revista Brasileira de Cirurgia Cardiovascular. 2014.

81. Nienaber JJ, Glower DD. Minitransseptal versus left atrial approach to the mitral valve: a comparison of outcomes. Ann Thorac Surg. 2006;82(3):834-839; discussion 839.

82. Kumar N, Saad E, Prabhakar G, De Vol E, Duran CM. Extended transseptal versus conventional left atriotomy: early postoperative study. Ann Thorac Surg. 1995;60(2):426-430.

83. Suzuki R, Watanabe T, Matsukawa M, Hiroshige K, Sata S, Koyanagi T. Sinus node artery-preserving superior transseptal approach: a simple technique. Annals of thoracic and cardiovascular surgery : official journal of the Association of Thoracic and Cardiovascular Surgeons of Asia. 2011;17(5):514-517.

84. Legare JF, Buth KJ, Arora RC, Murphy DA, Sullivan JA, Hirsch GM. The dome of the left atrium: an alternative approach for mitral valve repair. Eur J Cardiothorac Surg. 2003;23(3):272-276. 
85. Little S, Flynn M, Pettersson GB, Gillinov AM, Blackstone EH. Revisiting the dome approach for partial sternotomy/minimally invasive mitral valve surgery. Ann Thorac Surg. 2009;87(3):694-697.

86. Badhwar V, Rankin JS, Ad N, et al. Surgical Ablation of Atrial Fibrillation in the United States: Trends and Propensity Matched Outcomes. The Annals of thoracic surgery. 2017;104(2):493-500.

87. DeRose JJ, Mancini DM, Chang HL, et al. Pacemaker Implantation After Mitral Valve Surgery With Atrial Fibrillation Ablation. Journal of the American College of Cardiology. 2019;73(19):2427-2435.

88. Gillinov AM, Svensson LG. Ablation of atrial fibrillation with minimally invasive mitral surgery. Ann Thorac Surg. 2007;84(3):1041-1042.

89. Raanani E, Albage A, David TE, Yau TM, Armstrong S. The efficacy of the Cox/maze procedure combined with mitral valve surgery: a matched control study. European journal of cardio-thoracic surgery : official journal of the European Association for Cardio-thoracic Surgery. 2001;19(4):438-442.

90. Doukas G, Samani NJ, Alexiou C, et al. Left atrial radiofrequency ablation during mitral valve surgery for continuous atrial fibrillation: a randomized controlled trial. JAMA. 2005;294(18):2323-2329.

91. Benussi S, Cini R, Gaynor SL, Alfieri O, Calafiore AM. Bipolar Radiofrequency Maze Procedure Through a Transseptal Approach. The Annals of Thoracic Surgery. 2010;90(3):1025-1027.

92. Oueida F, Elawady MA, Eskander K. Radiofrequency ablation of atrial fibrillation during mitral valve surgery. Asian Cardiovasc Thorac Ann. 2014;22(7):807-810.

93. Mohr FW, Fabricius AM, Falk V, et al. Curative treatment of atrial fibrillation with intraoperative radiofrequency ablation: short-term and midterm results. J Thorac Cardiovasc Surg. 2002;123(5):919-927.

94. Krakor R, Chares M, Schneider J, Bernau H, Eichler I. Mid-term results of endoscopic mitral valve repair in combination with endocardial or epicardial ablation. European Journal of Cardio-Thoracic Surgery. 2011;40(3):e125-e129.

95. Voskoboinik A, Kalman JM, De Silva A, et al. Alcohol Abstinence in Drinkers with Atrial Fibrillation. New England Journal of Medicine. 2020;382(1):20-28.

96. Turkyilmaz S, Kavala AA. Comparison of Left Atriotomy and Superior Transseptal Approaches in Mitral Valve Surgery. The heart surgery forum. 2018;21(4):E318-E321.

97. Cho MS, Heo R, Jin X, et al. Sick Sinus Syndrome After the Maze Procedure Performed Concomitantly With Mitral Valve Surgery. Journal of the American Heart Association. 2018;7(19):e009629.

98. Saksena S, Hettrick DA, Koehler JL, Grammatico A, Padeletti L. Progression of paroxysmal atrial fibrillation to persistent atrial fibrillation in patients with bradyarrhythmias. American heart journal. 2007;154(5):884-892. 
99. Stulak JM, Sundt TM, III, Dearani JA, Daly RC, Orsulak TA, Schaff HV. Ten-year Experience With the Cox-Maze Procedure for Atrial Fibrillation: How Do We Define Success? The Annals of Thoracic Surgery. 2007;83(4):1319-1324.

100. Tiwari KK, Gasbarri T, Bevilacqua S, Glauber M. Right-Sided Minithoracotomy as a Surgical Approach for the Concomitant Treatment of Atrial Fibrillation. Res Cardiovasc Med. 2016;5(2):e31374-e31374.

101. Scherer M, Dzemali O, Aybek T, Wimmer-Greinecker G, Moritz A. Impact of left atrial size reduction on chronic atrial fibrillation in mitral valve surgery. The Journal of heart valve disease. 2003;12(4):469-474. 